\title{
One-Pot Four-Component Synthesis of 2-Aryl-3,3-Dihaloacrylonitriles using Potassium Hexacyanoferrate(II) as Environmentally Benign Cyanide Source
}

\author{
Zhouxing Zhao and Zheng $\mathrm{Li}^{*}$ \\ Key Laboratory of Polymer Materials of Gansu Province, College of Chemistry and Chemical \\ Engineering, Northwest Normal University, Lanzhou, Gansu, 730070, P. R. China
}

\begin{abstract}
Foi descrita uma rota eficiente para reações em uma etapa com quatro componentes incluindo cloretos de aroila, hexacianoferrato(II) de potássio, trifenilfosfina e tetrahaletos de carbono para sintetizar 2-aril-3,3-dicloroacrilonitrilas e 2-aril-3,3-dibromoacrilonitrilas. Este protocolo tem como vantagens o uso de uma fonte não-tóxica de cianeto, alto rendimento e procedimento experimental simples.
\end{abstract}

An efficient route to one-pot four-component reactions of aroyl chlorides, potassium hexacyanoferrate(II), triphenylphosphine and carbon tetrahalides to synthesize 2-aryl-3,3dichloroacrylonitriles and 2-aryl-3,3-dibromoacrylonitriles was described. This protocol has advantages of use of non-toxic cyanide source, high yield and simple work-up procedure.

Keywords: cyanation, four-component synthesis, potassium hexacyanoferrate(II), cyanide source, triphenylphosphine, 2-aryl-3,3-dichloroacrylonitriles, 2-aryl-3,3-dibromoacrylonitriles

\section{Introduction}

3,3-Dichloroacrylonitriles and 3,3-dibromoacrylonitriles are well-known as the most important synthetic intermediates. ${ }^{1}$ Although 2-aryl-3,3-dichloroacrylonitriles and 2-aryl-3,3-dibromoacrylonitriles could be prepared directly from phosphorus ylides with aroyl cyanides, ${ }^{2}$ the commercially available aroyl cyanides are limited and comparatively expensive. Especially, synthesis of aroyl cyanides had to utilize strong toxic reagents as original cyanide sources, such as $\mathrm{HgCN},{ }^{3} \mathrm{NaCN},{ }^{4} \mathrm{CuCN},{ }^{5}$ $\mathrm{KCN},{ }^{6}$ and $\mathrm{TMSCN},{ }^{7}$ which render the cyanation of aroyl chlorides unsafe and environmentally unfriendly. In addition, the corresponding phosphorus ylides, which are unstable to oxygen and moisture, also required to synthesize by reactions of triphenylphosphine with carbon tetrahalides prior to use. Therefore, there is a need to explore environmentally benign cyanating agents and simple procedure for the synthesis of 2-aryl-3,3dichloroacrylonitriles and 2-aryl-3,3-dibromoacrylonitriles.

Potassium hexacyanoferrate(II), $\mathrm{K}_{4}\left[\mathrm{Fe}(\mathrm{CN})_{6}\right]$, is non-toxic and is even used in the food industry for metal precipitation. In addition, it has been described as an antiagglutinating auxiliary for table salt $(\mathrm{NaCl})$. $\mathrm{K}_{4}\left[\mathrm{Fe}(\mathrm{CN})_{6}\right]$ is commercially available on a ton scale and

\footnotetext{
*e-mail: lizheng@nwnu.edu.cn
}

is even cheaper than $\mathrm{KCN}$. Very recently, $\mathrm{K}_{4}\left[\mathrm{Fe}(\mathrm{CN})_{6}\right]$ has been proved to be an efficient cyanide source for the cyanation of halogenated arenes and aroyl chlorides to prepare benzonitriles, ${ }^{8}$ and aroyl cyanides. ${ }^{9}$

Herein, we wish to report an efficient one-pot fourcomponent synthesis of 2-aryl-3,3-dichloroacrylonitriles and 2-aryl-3,3-dibromoacrylonitriles using potassium hexacyanoferrate(II) as an environmentally benign cyanide source.

\section{Results and Discussion}

Initially, the reaction of benzoyl chloride, potassium hexacyanoferrate(II), triphenylphosphine and carbon tetrachloride was selected as a model reaction to examine the feasibility of one-pot four-component synthesis of 2-phenyl-3,3-dichloroacrylonitrile under different conditions (Scheme 1). It was found that the optimal mole ratio of benzoyl chloride to potassium hexacyanoferrate(II) and triphenylphosphine was 1:0.2:2 for the reaction, and the excess carbon tetrachloride were required because it acted both as a reactant and a solvent in this reaction. The 0.2 equivalent of potassium hexacyanoferrate(II) used in the reaction indicated that a small excess of $\mathrm{CN}^{-}$was utilized in the reaction. The best yield was obtained by the procedure of first conducting the reaction of benzoyl chloride and potassium hexacyanoferrate(II) at $160^{\circ} \mathrm{C}$ for $3 \mathrm{~h}$, then further 
reacting the resulting mixture with triphenylphosphine and carbon tetrachloride at $80^{\circ} \mathrm{C}$ for $2 \mathrm{~h}$.

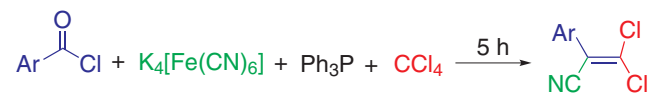

$$
\begin{aligned}
& \text { 1a-n }
\end{aligned}
$$

Scheme 1. One-pot synthesis of 2-aryl-3,3-dichloroacrylonitriles.

Under the optimal conditions, various substituted aroyl chlorides were examined for the one-pot four-component reactions. The results are summarized in Table 1. It was found that aroyl chlorides bearing electron-withdrawing substituents such as chloro, iodo and nitro groups on the aromatic ring gave the corresponding products in high yield (1b-1d, $\mathbf{1 i}, \mathbf{1 j}$ and $\mathbf{1 m})$. In contrast, aroyl chlorides bearing electron-donating substituents such as methyl, ethyl, methoxy and ethoxy groups on the aromatic ring gave the corresponding products in slightly lower yield under similar conditions (1e-1h, 1k and 1l). For ortho-substituted aroyl chlorides, the corresponding products were obtained in slightly lower yield than para-substituted ones, presumably due to the steric effect $(\mathbf{1 b}, \mathbf{1 e}$ and $\mathbf{1 h})$. Heteroaroyl chloride, furoyl chloride, was also very efficient for the one-pot fourcomponent reaction $(\mathbf{1 n})$.

In addition, one-pot four-component reactions of aroyl chlorides, potassium hexacyanoferrate(II), triphenylphosphine and carbon tetrabromide to synthesize various 2-aryl-3,3-dibromoacrylonitriles were also investigated (Scheme 2). In comparison with carbon tetrachloride, carbon tetrabromide is more active for the onepot four-component reaction. Therefore milder conditions are required, such as lower reaction temperature and short reaction time although solvent is needed because carbon tetrabromide can not act as a solvent at low temperatures. The optimal conditions for the selected four-component reaction of benzoyl chloride, potassium hexacyanoferrate(II), triphenylphosphine and carbon tetrabromide were also investigated. It was found that the optimal mole ratio of benzoyl chloride to potassium hexacyanoferrate(II), triphenylphosphine and carbon tetrabromide was 1:0.2:2:1 for the reaction, and the best yield was obtained by the procedure of first conducting the reaction of benzoyl chloride and potassium hexacyanoferrate(II) at $160{ }^{\circ} \mathrm{C}$ for $3 \mathrm{~h}$, then further reacting the resulting mixture with triphenylphosphine and carbon tetrabromide in methylene chloride at $10{ }^{\circ} \mathrm{C}$ for $0.5 \mathrm{~h}$.

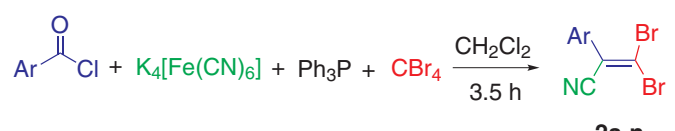

Scheme 2. One-pot synthesis of 2-aryl-3,3-dibromoacrylonitriles.
Table 1. One-pot four-component synthesis of various 2-aryl-3,3dichloroacrylonitriles

\begin{tabular}{lcccc}
\hline Compd. & $\begin{array}{c}\text { Aroyl } \\
\text { chloride }\end{array}$ & Product & $\begin{array}{c}\text { Yield }^{\mathrm{a}} \\
(\%)\end{array}$ & $\begin{array}{c}\mathrm{mp} \text { (lit.) } \\
\left({ }^{\circ} \mathrm{C}\right)\end{array}$ \\
\hline 12 & $\mathrm{CCl}_{2}$ & & Oil \\
& & &
\end{tabular}

$1 \mathbf{b}$<smiles>O=C(Cl)c1ccccc1Cl</smiles><smiles>N#CC(c1ccccc1Cl)C(Cl)(Cl)Cl</smiles>
78 104-105

$1 \mathrm{c}$<smiles>O=C(Cl)c1cccc(Cl)c1</smiles><smiles>N#CC(c1cccc(Cl)c1)C(Cl)(Cl)Cl</smiles>
80 54-56

$1 d$<smiles>O=C(Cl)c1ccc(Cl)cc1</smiles><smiles>N#CC(c1ccc(Cl)cc1)C(Cl)(Cl)Cl</smiles>

1e<smiles>Cc1ccccc1C(=O)Cl</smiles><smiles>Cc1ccccc1C(C#N)C(Cl)(Cl)Cl</smiles>

Oil

1f<smiles>Cc1cccc(C(=O)Cl)c1</smiles><smiles>Cc1cccc(C(Cl)(C#N)C(Cl)(Cl)Cl)c1</smiles>
71 $(\mathrm{Oil})^{2}$

19<smiles>Cc1ccc(C(=O)Cl)cc1</smiles><smiles>Cc1ccc(C(C#N)C(Cl)(Cl)Cl)cc1</smiles>

Oil

$1 h$<smiles>Cc1ccccc1C(=O)Cl</smiles><smiles>CCC(C#N)c1ccccc1O</smiles>

Oil

1i<smiles>O=C(Cl)c1cccc([N+](=O)[O-])c1</smiles><smiles>N#CC(c1cccc([N+](=O)[O-])c1)C(Cl)(Cl)Cl</smiles>
79

137-139

1j<smiles>O=C(Cl)c1ccc([N+](=O)[O-])cc1</smiles><smiles>N#CC(c1ccc([N+](=O)[O-])cc1)C(Cl)(Cl)Cl</smiles>

128-130

$1 k$<smiles>COc1ccc(C(=O)Cl)cc1</smiles><smiles>COc1ccc(C(Cl)(C#N)C(Cl)(Cl)Cl)cc1</smiles>
75 102-104

11<smiles>COc1ccc(C(C)=O)cc1</smiles><smiles>COc1ccc(C(C#N)C(Cl)(Cl)Cl)cc1</smiles><smiles>O=C(Cl)c1ccc(I)cc1</smiles><smiles>N#CC(c1ccc(I)cc1)C(Cl)(Cl)Cl</smiles>
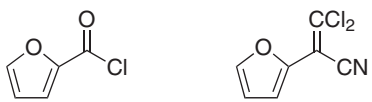

100-102

\footnotetext{
${ }^{a}$ Isolated yields.
} 
Under the optimal conditions, various substituted aroyl chlorides were examined for the reactions. The results are summarized in Table 2. The various substituted aroyl chlorides including electron-withdrawing groups and electron-donating groups are effective for the reactions, which have the similar effect on the yield to carbon tetrachloride. It is noteworthy to mention that 4-phenylbenzoyl chloride and isophthaloyl dichloride could also efficiently participate in the one-pot fourcomponent reactions of carbon tetrabromide, but not for the reactions of carbon tetrachloride under the studied conditions (2l and $\mathbf{2 m}$ ). Heteroaroyl chloride, furoyl chloride, was also very efficient for the one-pot four-component reaction to obtain 2-(furan-2-yl)-3,3dibromoacrylonitrile (2n).

\section{Conclusions}

An efficient route to the one-pot four-component reactions of aroyl chlorides, potassium hexacyanoferrate(II), triphenylphosphine and carbon tetrahalides to synthesize 2-aryl-3,3-dichloroacrylonitriles and 2-aryl3,3-dibromoacrylonitriles has been developed. The protocol has the advantages of using non-toxic potassium hexacyanoferrate(II) as an environmentally benign cyanide source instead of traditional strong toxic cyanating agents, high yield and simple work-up procedure.

\section{Experimental}

IR spectra were recorded using $\mathrm{KBr}$ pellets on an Alpha Centauri FTIR spectrophotometer and ${ }^{1} \mathrm{H}$ NMR and ${ }^{13} \mathrm{C}$ NMR spectra on a Mercury-400BB instrument using $\mathrm{CDCl}_{3}$ as solvent and $\mathrm{Me}_{4} \mathrm{Si}$ as internal standard. Melting points were observed in an electrothermal melting point apparatus. Potassium hexacyanoferrate(II) dried at $80^{\circ} \mathrm{C}$ under vacuum for $24 \mathrm{~h}$ and finely powdered prior to use. All reactions were monitored by TLC. Flash column chromatography was carried out using 200-300 mesh silica gel at increased pressure.

General procedure for the preparation of 2-aryl-3,3dichloroacrylonitriles

The mixture of aroyl chloride $(10 \mathrm{mmol})$ and potassium hexacyanoferrate(II) $(0.84 \mathrm{~g}, 2 \mathrm{mmol})$ was heated at $160{ }^{\circ} \mathrm{C}$ for $3 \mathrm{~h}$. After cooling to room temperature, triphenylphosphine $(5.24 \mathrm{~g}, 20 \mathrm{mmol})$ and carbon tetrachloride $(10 \mathrm{~mL})$ were added. The resulting mixture was further stirred at $80{ }^{\circ} \mathrm{C}$ for $2 \mathrm{~h}$. Then the solid was removed by filtration, and the filtrate was evaporated to
Table 2. One-pot four-component synthesis of various 2-aryl-3,3dibromoacrylonitriles

\begin{tabular}{|c|c|c|c|c|}
\hline Compd. & Aroyl chloride & Product & $\begin{array}{l}\text { Yield }^{\mathrm{a}} \\
(\%)\end{array}$ & $\begin{array}{l}\mathrm{mp} \\
\left({ }^{\circ} \mathrm{C}\right)\end{array}$ \\
\hline $2 a$ & & & 75 & $62-64$ \\
\hline $2 b$ & & & 78 & $84-86$ \\
\hline $2 c$ & & & 80 & $78-80$ \\
\hline $2 d$ & & & 82 & 103-104 \\
\hline $2 e$ & & & 72 & $74-76$ \\
\hline $2 f$ & & & 75 & $40-41$ \\
\hline $2 \mathrm{~g}$ & & & 77 & $74-76$ \\
\hline $2 \mathrm{~h}$ & & & 80 & $130-132$ \\
\hline $2 \mathrm{i}$ & & & 83 & $88-90$ \\
\hline $2 j$ & & & 78 & $158-160$ \\
\hline $2 k$ & & & 77 & $136-138$ \\
\hline 21 & & & 68 & $74-76$ \\
\hline $2 m$ & & & 84 & $156-158$ \\
\hline $2 n$ & & & 82 & $52-53$ \\
\hline
\end{tabular}

${ }^{\mathrm{a}}$ Isolated yields. 
remove solvent under reduced pressure, and the residue was subjected to silica gel flash column chromatography (ethyl acetate, petroleum ether, 1:40, v/v) to obtain pure product. The analytical and spectral data for products are given below; melting poinst are given in Table 1 .

\section{2-Phenyl-3,3-dichloroacrylonitrile (1a)}

Colorless oil; ${ }^{1} \mathrm{H}$ NMR $\left(\mathrm{CDCl}_{3}, 400 \mathrm{MHz}\right): \delta$ 7.52$7.41(\mathrm{~m}, 5 \mathrm{H}, \mathrm{Ar}-\mathrm{H}) ;{ }^{13} \mathrm{C} \mathrm{NMR}\left(\mathrm{CDCl}_{3}, 100 \mathrm{MHz}\right): \delta$ $137.1,130.6,130.0,128.8,128.6,115.5,108.2$; IR $(\mathrm{KBr})$ $\mathrm{v}_{\max } / \mathrm{cm}^{-1}: 3061,2922,2223,1563,1445,930,824,759$, 695. Found: C, 54.65; H, 2.53; N, 7.10. Calc. for $\mathrm{C}_{9} \mathrm{H}_{5} \mathrm{Cl}_{2} \mathrm{~N}$ : C, 54.58; H, 2.54; N, 7.07\%.

\section{2-(2-Chlorophenyl)-3,3-dichloroacrylonitrile (1b)}

White solid; ${ }^{1} \mathrm{H} \mathrm{NMR}\left(\mathrm{CDCl}_{3}, 400 \mathrm{MHz}\right): \delta 7.60-7.31$ $(\mathrm{m}, 4 \mathrm{H}, \mathrm{Ar}-\mathrm{H}),{ }^{13} \mathrm{C} \mathrm{NMR}\left(\mathrm{CDCl}_{3}, 100 \mathrm{MHz}\right): \delta 132.9$, 132.4, 131.5, 130.4, 130.2, 127.4, 120.4, 115.6, 113.9; IR $(\mathrm{KBr}) v_{\max } / \mathrm{cm}^{-1}: 3042,2999,2219,1604,1510,1256,927$, 825. Found: C, $46.55 ; \mathrm{H}, 1.72 ; \mathrm{N}, 6.04$. Calc. for $\mathrm{C}_{9} \mathrm{H}_{4} \mathrm{Cl}_{3} \mathrm{~N}$ : C, 46.49; H, 1.73; N, $6.02 \%$.

\section{2-(3-Chlorophenyl)-3,3-dichloroacrylonitrile (1c)}

Light yellow solid; ${ }^{1} \mathrm{H}$ NMR $\left(\mathrm{CDCl}_{3}, 400 \mathrm{MHz}\right): \delta$ 7.52 (s, 1H, Ar-H), 7.44-7.39 (m, 3H, Ar-H); ${ }^{13} \mathrm{C}$ NMR $\left(\mathrm{CDCl}_{3}, 100 \mathrm{MHz}\right): \delta 138.6,134.9,132.2,130.3,130.2$, $128.8,126.9,115.1,114.3$; IR (KBr) $v_{\max } / \mathrm{cm}^{-1}: 3062,2926$, 2220, 1571, 1471, 1262, 943, 850. Found: C, 46.42; H, 1.73; $\mathrm{N}, 5.99$. Calc. for $\mathrm{C}_{9} \mathrm{H}_{4} \mathrm{Cl}_{3} \mathrm{~N}$ : C, 46.49; $\mathrm{H}, 1.73 ; \mathrm{N}, 6.02 \%$.

\section{2-(4-Chlorophenyl)-3,3-dichloroacrylonitrile (1d)}

Light yellow solid; ${ }^{1} \mathrm{H}$ NMR $\left(\mathrm{CDCl}_{3}, 400 \mathrm{MHz}\right): \delta$ 7.48-7.40 (m, 4H, Ar-H); $\left.{ }^{13} \mathrm{C} \mathrm{NMR} \mathrm{(CDCl}, 100 \mathrm{MHz}\right): \delta$ $137.7,136.2$, 130.0, 129.1, 129.0, 115.2, 114.5; IR (KBr) $v_{\max } / \mathrm{cm}^{-1}: 3084,2924,2220,1555,1481,1279,927,809$. Found: C, 46.45; $\mathrm{H}, 1.72 ; \mathrm{N}, 6.01$. Calc. for $\mathrm{C}_{9} \mathrm{H}_{4} \mathrm{Cl}_{3} \mathrm{~N}$ : C, 46.49; H, 1.73; N, 6.02\%.

\section{2-(2-Methylphenyl)-3,3-dichloroacrylonitrile (1e)}

Colorless oil; ${ }^{1} \mathrm{H}$ NMR $\left(\mathrm{CDCl}_{3}, 400 \mathrm{MHz}\right): \delta 7.22-7.00$ (m, 4H, Ar-H), $2.16\left(\mathrm{~s}, 3 \mathrm{H}, \mathrm{CH}_{3}\right) ;{ }^{13} \mathrm{C} \mathrm{NMR}\left(\mathrm{CDCl}_{3}, 100\right.$ $\mathrm{MHz}): \delta 138.5,136.1,130.4,130.0,129.9,128.7,126.2$, 114.7, 114.2, 18.8; IR (KBr) $v_{\max } / \mathrm{cm}^{-1}: 3067,2925,2219$, 1571, 1485, 1258, 930, 753. Found: C, 56.53; H, 3.32; N, 6.62. Calc. for $\mathrm{C}_{10} \mathrm{H}_{7} \mathrm{Cl}_{2} \mathrm{~N}$ : C, 56.63; H, 3.33; N, 6.60\%.

\section{2-(3-Methylphenyl)-3,3-dichloroacrylonitrile (1f)}

Colorless oil; ${ }^{1} \mathrm{HNMR}\left(\mathrm{CDCl}_{3}, 400 \mathrm{MHz}\right): \delta 7.28-7.20(\mathrm{~m}$, $4 \mathrm{H}, \mathrm{Ar}-\mathrm{H}), 2.35$ (s, 3H, $\left.\mathrm{CH}_{3}\right) ;{ }^{13} \mathrm{C} \mathrm{NMR}\left(\mathrm{CDCl}_{3}, 100 \mathrm{MHz}\right): \delta$ 138.5, 136.6, 130.6, 130.4, 128.9, 128.5, 125.5, 115.5, 115.4, 21.0; IR (KBr) $v_{\max } / \mathrm{cm}^{-1}: 3039,2923,2222,1565,1485,1267$,
931, 877, 791, 697. Found: C, 56.56; H, 3.34; N, 6.56. Calc. for $\mathrm{C}_{10} \mathrm{H}_{7} \mathrm{Cl}_{2} \mathrm{~N}$ : C, 56.63; H, 3.33; N, 6.60\%.

\section{2-(4-Methylphenyl)-3,3-dichloroacrylonitrile (1g)}

Colorless oil; ${ }^{1} \mathrm{H}$ NMR $\left(\mathrm{CDCl}_{3}, 400 \mathrm{MHz}\right): \delta 7.37$ (d, 2H, J $8.4 \mathrm{~Hz}, \mathrm{Ar}-\mathrm{H}), 7.19$ (d, 2H, J $8.4 \mathrm{~Hz}, \mathrm{Ar}-\mathrm{H}), 2.32$

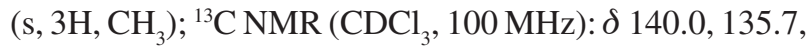
129.2, 128.2, 127.4, 115.3, 115.2, 20.9; IR (KBr) $v_{\max } / \mathrm{cm}^{-1}$ : 3031, 2923, 2222, 1573, 1509, 1258, 929, 815, 771. Found: C, 56.70; H, 3.32; N, 6.57. Calc. for $\mathrm{C}_{10} \mathrm{H}_{7} \mathrm{Cl}_{2} \mathrm{~N}$ : C, 56.63; H, 3.33; N, $6.60 \%$.

\section{2-(2-Ethylphenyl)-3,3-dichloroacrylonitrile (1h)}

Colorless oil; ${ }^{1} \mathrm{H} \mathrm{NMR}\left(\mathrm{CDCl}_{3}, 400 \mathrm{MHz}\right): \delta 7.25-6.97$ (m, 4H, Ar-H), 2.88 (q, 2H, J $6.4 \mathrm{~Hz}, \mathrm{CH}_{2}$ ), 1.26 (t, 3H, $\left.J 6.4 \mathrm{~Hz}, \mathrm{CH}_{3}\right) ;{ }^{13} \mathrm{C} \mathrm{NMR}\left(\mathrm{CDCl}_{3}, 100 \mathrm{MHz}\right): \delta 143.1$, 129.1, 129.0, 128.8, 124.7, 124.1, 121.6, 120.3, 119.2, 25.6, 14.3; IR (KBr) v $v_{\max } / \mathrm{cm}^{-1}: 3032,2918,2224,1589,1497$, 1234, 942, 842. Found: C, 58.50; H, 3.99; N, 6.20. Calc. for $\mathrm{C}_{11} \mathrm{H}_{9} \mathrm{Cl}_{2} \mathrm{~N}$ : C, 58.43; H, 4.01; N, 6.19\%.

\section{2-(3-Nitrophenyl)-3,3-dichloroacrylonitrile (1i)}

White solid; ${ }^{1} \mathrm{H} \mathrm{NMR}\left(\mathrm{CDCl}_{3}, 400 \mathrm{MHz}\right): \delta 8.45$ (s, $1 \mathrm{H}$, Ar-H), 8.33 (d, 1H, J $8.0 \mathrm{~Hz}, \mathrm{Ar}-\mathrm{H}), 7.87$ (d, 1H, J8.0 Hz, $\mathrm{Ar}-\mathrm{H}), 7.70$ (t, $1 \mathrm{H}, J 8.0 \mathrm{~Hz}, \mathrm{Ar}-\mathrm{H}) ;{ }^{13} \mathrm{C} \mathrm{NMR}\left(\mathrm{CDCl}_{3}, 100\right.$ $\mathrm{MHz}): \delta$ 148.2, 140.1, 134.6, 132.2, 130.2, 124.9, 123.9, 114.7, 113.4; IR (KBr) $v_{\max } / \mathrm{cm}^{-1}: 3081,2924,2223,1614$, 1532, 1348, 1263, 947. Found: C, 44.55; H, 1.67; N, 11.57. Calc. for $\mathrm{C}_{9} \mathrm{H}_{4} \mathrm{Cl}_{2} \mathrm{~N}_{2} \mathrm{O}_{2}: \mathrm{C}, 44.48 ; \mathrm{H}, 1.66 ; \mathrm{N}, 11.53 \%$.

\section{2-(4-Nitrophenyl)-3,3-dichloroacrylonitrile (1j)}

White solid; ${ }^{1} \mathrm{H}$ NMR $\left(\mathrm{CDCl}_{3}, 400 \mathrm{MHz}\right): \delta 8.33$ (d, $J$ $9.2 \mathrm{~Hz}, 2 \mathrm{H}, \mathrm{Ar}-\mathrm{H}), 7.74$ (d, 2H, J9.2 Hz, Ar-H); ${ }^{13} \mathrm{C}$ NMR $\left(\mathrm{CDCl}_{3}, 100 \mathrm{MHz}\right): \delta$ 148.3, 140.2, 136.7, 130.0, 124.1, 114.7, 113.8; IR (KBr) $v_{\max } / \mathrm{cm}^{-1}: 3049,2923,2235,1555$, 1481, 1377, 1226, 1024, 939, 748. Found: C, 44.45; H, 1.66; N, 11.49. Calc. for $\mathrm{C}_{9} \mathrm{H}_{4} \mathrm{Cl}_{2} \mathrm{~N}_{2} \mathrm{O}_{2}$ : C, 44.48; $\mathrm{H}, 1.66$; $\mathrm{N}, 11.53 \%$.

\section{2-(4-Ethoxyphenyl)-3,3-dichloroacrylonitrile (1k)}

White solid; ${ }^{1} \mathrm{H} \mathrm{NMR}\left(\mathrm{CDCl}_{3}, 400 \mathrm{MHz}\right): \delta 7.46(\mathrm{~d}$, $2 \mathrm{H}, J 8.8 \mathrm{~Hz}, \mathrm{Ar}-\mathrm{H}), 6.93$ (d, 2H, J $8.8 \mathrm{~Hz}, \mathrm{Ar}-\mathrm{H}), 4.06$ (q, $\left.2 \mathrm{H}, J 6.8 \mathrm{~Hz}, \mathrm{CH}_{2}\right), 1.43$ (t, 3H, J $\left.6.8 \mathrm{~Hz}, \mathrm{CH}_{3}\right) ;{ }^{13} \mathrm{C} \mathrm{NMR}$ $\left(\mathrm{CDCl}_{3}, 100 \mathrm{MHz}\right): \delta$ 160.0, 135.3, 130.2, 122.6, 115.8, 115.2, 114.6, 63.6, 14.6; IR (KBr) $v_{\max } / \mathrm{cm}^{-1}: 3059,2988$, 2218, 1606, 1510, 1265, 924. Found: C, 54.50; H, 3.77; N, 5.80. Calc. for $\mathrm{C}_{11} \mathrm{H}_{9} \mathrm{Cl}_{2} \mathrm{NO}$ : C, 54.57; H, 3.75; N, 5.79\%.

\section{2-(4-Methoxyphenyl)-3,3-dichloroacrylonitrile (1l)}

White solid; ${ }^{1} \mathrm{H}$ NMR $\left(\mathrm{CDCl}_{3}, 400 \mathrm{MHz}\right): \delta 7.48(\mathrm{~d}$, 2H, J 9.2 Hz, Ar-H), 6.95 (d, 2H, J 9.2 Hz, Ar-H), 3.84 
(s, $\left.3 \mathrm{H}, \mathrm{CH}_{3}\right) ;{ }^{13} \mathrm{C} \mathrm{NMR}\left(\mathrm{CDCl}_{3}, 100 \mathrm{MHz}\right): \delta 160.7,135.4$, $130.2,122.8,115.7,115.2,114.2,55.3 ; \mathrm{IR}(\mathrm{KBr}) \mathrm{v}_{\max } / \mathrm{cm}^{-1}$ : 3067, 2988, 2218, 1589, 1465, 1287, 854, 765. Found: C, 52.59; $\mathrm{H}, 3.07 ; \mathrm{N}, 6.17$. Calc. for $\mathrm{C}_{10} \mathrm{H}_{7} \mathrm{Cl}_{2} \mathrm{NO}$ : C, 52.66; $\mathrm{H}, 3.09$; $\mathrm{N}, 6.14 \%$.

\section{2-(4-Iodophenyl)-3,3-dichloroacrylonitrile (1m)}

White solid; ${ }^{1} \mathrm{H}$ NMR $\left(\mathrm{CDCl}_{3}, 400 \mathrm{MHz}\right): \delta 7.79(\mathrm{~d}, 2 \mathrm{H}$, $J 8.8 \mathrm{~Hz}, \mathrm{Ar}-\mathrm{H}), 7.26$ (d, 2H, $J 8.8 \mathrm{~Hz}, \mathrm{Ar}-\mathrm{H}) ;{ }^{13} \mathrm{C}$ NMR $\left(\mathrm{CDCl}_{3}, 100 \mathrm{MHz}\right): \delta 138.1,137.8,130.3,130.1,115.2$, 114.7, 96.6; IR (KBr) $v_{\text {max }} / \mathrm{cm}^{-1}: 3065,2220,1572,1481$, 1288, 1276, 933, 834. Found: C, 33.41; H, 1.23; N, 4.31. Calc. for $\mathrm{C}_{9} \mathrm{H}_{4} \mathrm{Cl}_{2} \mathrm{IN}$ : C, 33.37; H, 1.24; N, 4.32\%.

\section{2-(Furan-2-yl)-3,3-dichloroacrylonitrile (1n)}

White solid; ${ }^{1} \mathrm{H}$ NMR $\left(\mathrm{CDCl}_{3}, 400 \mathrm{MHz}\right): \delta 7.76$ (d, $1 \mathrm{H}, J 1.6 \mathrm{~Hz}, \mathrm{Fu}-\mathrm{H}), 7.04$ (d, 1H, J 4.4 Hz, Fu-H), 6.41 $(\mathrm{dd}, 1 \mathrm{H}, J 3.6 \mathrm{~Hz}, J 1.6 \mathrm{~Hz}, \mathrm{Fu}-\mathrm{H}),{ }^{13} \mathrm{C}$ NMR $\left(\mathrm{CDCl}_{3}, 100\right.$ MHz): $\delta$ 148.3, 140.2, 136.6, 130.0, 124.1, 114.7, 113.8; IR (KBr) $v_{\text {max }} / \mathrm{cm}^{-1}: 3109,2923,2220,1520,1350,931$, 858, 817, 698. Found: C, 44.66; H, 1.62; N, 7.43. Calc. for $\mathrm{C}_{7} \mathrm{H}_{3} \mathrm{Cl}_{2} \mathrm{NO}: \mathrm{C}, 44.72 ; \mathrm{H}, 1.61 ; \mathrm{N}, 7.45 \%$.

General procedure for the preparation of 2-aryl-3,3dibromoacrylonitriles

The mixture of aroyl chloride $(10 \mathrm{mmol})$ and potassium hexacyanoferrate(II) $(0.84 \mathrm{~g}, 2 \mathrm{mmol})$ was heated at $160{ }^{\circ} \mathrm{C}$ for $3 \mathrm{~h}$. After cooling to $10{ }^{\circ} \mathrm{C}$, triphenylphosphine $(5.24 \mathrm{~g}, 20 \mathrm{mmol})$ and carbon tetrabromide $(3.31 \mathrm{~g}, 10 \mathrm{mmol})$ in $10 \mathrm{~mL}$ of methylene chloride was slowly added dropwise with stirring. The resulting mixture was further stirred at $10{ }^{\circ} \mathrm{C}$ for $0.5 \mathrm{~h}$. Then the solid was removed by filtration, and the filtrate was evaporated to remove solvent under reduced pressure, and the residue was subjected to silica gel flash column chromatography (ethyl acetate, petroleum ether, $1: 40, \mathrm{v} / \mathrm{v}$ ) to obtain pure product. The analytical and spectral data for products are given below; melting points are given in Table 2.

\section{2-Phenyl-3,3-dibromoacrylonitrile (2a)}

White solid; ${ }^{1} \mathrm{H} \mathrm{NMR}\left(\mathrm{CDCl}_{3}, 400 \mathrm{MHz}\right): \delta$ 7.48-7.45 (m, 5H, Ar-H); ${ }^{13} \mathrm{C}$ NMR $\left(\mathrm{CDCl}_{3}, 100 \mathrm{MHz}\right): \delta 133.3$, $130.0,128.9,128.5,122.6,117.0,109.7 ; \mathrm{IR}(\mathrm{KBr}) \mathrm{v}_{\max } / \mathrm{cm}^{-1}$ : 3062, 2923, 2216, 1443, 842. Found: C, 37.71; H, 1.77; $\mathrm{N}, 4$.86. Calc. for $\mathrm{C}_{9} \mathrm{H}_{5} \mathrm{Br}_{2} \mathrm{~N}$ : C, 37.67; H, 1.76; N, $4.88 \%$.

\section{2-(2-Chlorophenyl)-3,3-dibromoacrylonitrile (2b)}

White solid; ${ }^{1} \mathrm{H}$ NMR $\left(\mathrm{CDCl}_{3}, 400 \mathrm{MHz}\right): \delta 7.50-7.31$ (m, 4H, Ar-H); ${ }^{13} \mathrm{C}$ NMR $\left(\mathrm{CDCl}_{3}, 100 \mathrm{MHz}\right): \delta 132.8$,
132.4, 131.5, 130.4, 130.2, 127.4, 120.3, 115.5, 113.9; IR $(\mathrm{KBr}) \mathrm{v}_{\max } / \mathrm{cm}^{-1}: 3069,2924,2218,1554,1467,11435$, 1287,1038, 853, 750. Found: C, 33.69; H, 1.25; N, 4.34. Calc. for $\mathrm{C}_{9} \mathrm{H}_{4} \mathrm{Br}{ }_{2} \mathrm{ClN}$ : C, 33.63; H, $1.25 ; \mathrm{N}, 4.36 \%$.

\section{2-(3-Chlorophenyl)-3,3-dibromoacrylonitrile (2c)}

White solid; ' ${ }^{1} \mathrm{H}$ NMR ( $\left.\mathrm{CDCl}_{3}, 400 \mathrm{MHz}\right): \delta$ 7.48-7.35 (m, 4H, Ar-H); ${ }^{13} \mathrm{C}$ NMR $\left(\mathrm{CDCl}_{3}, 100 \mathrm{MHz}\right): \delta 134.9$, 134.7, 130.3, 130.2, 128.6, 126.7, 121.2, 116.6, 111.1; IR $(\mathrm{KBr}) v_{\max } / \mathrm{cm}^{-1}: 3065,2927,2218,1589,1434,1282$, 981, 848, 751. Found: C, 33.59; H, 1.26; N, 4.35. Calc. for $\mathrm{C}_{9} \mathrm{H}_{4} \mathrm{Br}_{2} \mathrm{ClN}$ : C, 33.63; H, 1.25; N, 4.36\%.

\section{2-(4-Chlorophenyl)-3,3-dibromoacrylonitrile (2d)}

White solid; ${ }^{1} \mathrm{H}$ NMR $\left(\mathrm{CDCl}_{3}, 400 \mathrm{MHz}\right): \delta 7.43$ (s, $4 \mathrm{H}, \mathrm{Ar}-\mathrm{H}) ;{ }^{13} \mathrm{C}$ NMR $\left(\mathrm{CDCl}_{3}, 100 \mathrm{MHz}\right): \delta$ 136.2, 131.6, $129.9,129.2,121.5,116.7,110.4$; IR (KBr) $v_{\text {max }} / \mathrm{cm}^{-1}: 2922$, $2213,1590,1482,1095,831$. Found: $\mathrm{C}, 33.65 ; \mathrm{H}, 1.25 ; \mathrm{N}$, 4.38. Calc. for $\mathrm{C}_{9} \mathrm{H}_{4} \mathrm{Br}_{2} \mathrm{ClN}$ : C, 33.63; $\mathrm{H}, 1.25 ; \mathrm{N}, 4.36 \%$.

\section{2-(2-Methylphenyl)-3,3-dibromoacrylonitrile (2e)}

White solid; ${ }^{1} \mathrm{H}$ NMR $\left(\mathrm{CDCl}_{3}, 400 \mathrm{MHz}\right): \delta 7.37-7.18$ (m, 4H, Ar-H), 2.33 (s, 3H, $\left.\mathrm{CH}_{3}\right) ;{ }^{13} \mathrm{C} \mathrm{NMR}\left(\mathrm{CDCl}_{3}, 100\right.$ $\mathrm{MHz}): \delta 136.1,133.3,130.8,130.2,128.8,126.6,122.4$, 116.1, 111.9, 19.3; IR (KBr) $v_{\max } / \mathrm{cm}^{-1}: 3062,2920,2214$, 1546, 1453, 1251, 852, 735. Found: C, 39.86; H, 2.35; N, 4.63. Calc. for $\mathrm{C}_{10} \mathrm{H}_{7} \mathrm{Br}_{2} \mathrm{~N}$ : C, 39.91; H, 2.34; $\mathrm{N}, 4.65 \%$.

\section{2-(3-Methylphenyl)-3,3-dibromoacrylonitrile (2f)}

White solid; ${ }^{1} \mathrm{H}$ NMR $\left(\mathrm{CDCl}_{3}, 400 \mathrm{MHz}\right): \delta$ 7.34-7.24 (m, 4H, Ar-H), 2.39 (s, $\left.3 \mathrm{H}, \mathrm{CH}_{3}\right) ;{ }^{13} \mathrm{C}$ NMR $\left(\mathrm{CDCl}_{3}, 100\right.$ MHz): $\delta$ 138.8, 133.2, 130.8, 128.9, 128.8, 125.6, 122.7, 117.1, 109.4, 21.3; IR (KBr) $v_{\max } / \mathrm{cm}^{-1}: 3018,2921,2214$, 1600, 1554, 1095, 850, 789. Found: C, 39.95; H, 2.34; N, 4.67. Calc. for $\mathrm{C}_{10} \mathrm{H}_{7} \mathrm{Br}_{2} \mathrm{~N}$ : C, 39.91; H, 2.34; N, $4.65 \%$.

\section{2-(4-Methylphenyl)-3,3-dibromoacrylonitrile (2g)}

White solid; ${ }^{1} \mathrm{H}$ NMR $\left(\mathrm{CDCl}_{3}, 400 \mathrm{MHz}\right): \delta$ 7.39-7.23 (m, 4H, Ar-H), 2.38 (s, 3H, $\left.\mathrm{CH}_{3}\right) ;{ }^{13} \mathrm{C}$ NMR $\left(\mathrm{CDCl}_{3}, 100\right.$ MHz): $\delta 140.4,130.4,129.6,128.4,122.6,117.1,108.9$, 21.4; IR (KBr) $v_{\max } / \mathrm{cm}^{-1}: 3028,2917,2220,1613,1551$, 1505, 1260, 849, 823. Found: C, 39.96; H, 2.35; N, 4.66. Calc. for $\mathrm{C}_{10} \mathrm{H}_{7} \mathrm{Br}_{2} \mathrm{~N}$ : C, 39.91; H, 2.34; N, 4.65\%.

\section{2-(4-Nitrophenyl)-3,3-dibromoacrylonitrile (2h)}

White solid; ${ }^{1} \mathrm{H}$ NMR $\left(\mathrm{CDCl}_{3}, 400 \mathrm{MHz}\right): \delta 8.32(\mathrm{~d}, 2 \mathrm{H}$, $J 7.2 \mathrm{~Hz}, \mathrm{Ar}-\mathrm{H}), 7.71(\mathrm{~d}, 2 \mathrm{H}, J 7.2 \mathrm{~Hz}, \mathrm{Ar}-\mathrm{H}) ;{ }^{13} \mathrm{C}$ NMR $\left(\mathrm{CDCl}_{3}, 100 \mathrm{MHz}\right): \delta$ 148.3, 139.1, 129.9, 124.2, 120.6, $116.2,112.7$; IR $(\mathrm{KBr}) v_{\max } / \mathrm{cm}^{-1}: 3104,2924,2216,1600$, 1518, 1350, 1294, 858. Found: C, 32.49; H, 1.21; N, 8.41. Calc. for $\mathrm{C}_{9} \mathrm{H}_{4} \mathrm{Br}_{2} \mathrm{~N}_{2} \mathrm{O}_{2}: \mathrm{C}, 32.56 ; \mathrm{H}, 1.21 ; \mathrm{N}, 8.44 \%$. 
2-(3,5-Dinitrophenyl)-3,3-dibromoacrylonitrile (2i)

White solid; ${ }^{1} \mathrm{H} \mathrm{NMR}\left(\mathrm{CDCl}_{3}, 400 \mathrm{MHz}\right): \delta 9.13(\mathrm{~s}, 1 \mathrm{H}$, $\mathrm{Ar}-\mathrm{H}), 8.74$ (s, 2H, $\mathrm{Ar}-\mathrm{H}) ;{ }^{13} \mathrm{C}$ NMR $\left(\mathrm{CDCl}_{3}, 100 \mathrm{MHz}\right): \delta$ 148.7, 136.3, 129.0, 128.9, 120.0, 118.4, 115.6; IR (KBr) $v_{\max } / \mathrm{cm}^{-1}: 3094,2983,2224,1627,1543,1344,1279,918$, 853, 729. Found: C, 28.73; H, 0.80; N, 11.11. Calc. for $\mathrm{C}_{9} \mathrm{H}_{3} \mathrm{Br}_{2} \mathrm{~N}_{3} \mathrm{O}_{4}: \mathrm{C}, 28.68 ; \mathrm{H}, 0.80 ; \mathrm{N}, 11.15 \%$.

\section{2-(3-Nitrophenyl)-3,3-dibromoacrylonitrile (2j)}

White solid; ${ }^{1} \mathrm{H}$ NMR $\left(\mathrm{CDCl}_{3}, 400 \mathrm{MHz}\right): \delta 8.41$ (s, $1 \mathrm{H}, \mathrm{Ar}-\mathrm{H}), 8.33(\mathrm{~d}, 1 \mathrm{H}, J 8.4 \mathrm{~Hz}, \mathrm{Ar}-\mathrm{H}), 7.84(\mathrm{~d}, 1 \mathrm{H}, J$ $8.4 \mathrm{~Hz}, \mathrm{Ar}-\mathrm{H}), 7.69$ (t, 1H, J 8.4 Hz, Ar-H); ${ }^{13} \mathrm{C}$ NMR $\left(\mathrm{CDCl}_{3}, 100 \mathrm{MHz}\right): \delta 148.3,134.7,134.5,130.3,124.8$, 123.8, 120.3, 116.2, 112.8; IR (KBr) $v_{\max } / \mathrm{cm}^{-1}: 3104$, 2924, 2216, 1600, 1518, 1350, 1294, 858. Found: C, 32.61; $\mathrm{H}, 1.20 ; \mathrm{N}$, 8.46. Calc. for $\mathrm{C}_{9} \mathrm{H}_{4} \mathrm{Br}_{2} \mathrm{~N}_{2} \mathrm{O}_{2}$ : C, 32.56; $\mathrm{H}, 1.21 ; \mathrm{N}, 8.44 \%$.

\section{2-(4-Methoxyphenyl)-3,3-dibromoacrylonitrile ( $2 \boldsymbol{k})$}

IR ${ }^{1} \mathrm{H}$ NMR ( $\left.\mathrm{CDCl}_{3}, 400 \mathrm{MHz}\right): \delta 7.44$ (d, 2H, J 9.2 $\mathrm{Hz}, \mathrm{Ar}-\mathrm{H}), 6.94$ (d, 2H, J 9.2 Hz, Ar-H), 3.84 (s, 3H, $\left.\mathrm{CH}_{3}\right) \cdot{ }^{13} \mathrm{C} \mathrm{NMR}\left(\mathrm{CDCl}_{3}, 100 \mathrm{MHz}\right): \delta 160.6,130.1,125.4$, $122.2,117.1,114.2,108.0,55.3$. (KBr) $v_{\max } / \mathrm{cm}^{-1}: 2999$, 2939, 2214, 1605, 1508, 1259, 1182, 1021, 829. Found: C, 37.95; H, 2.22; N, 4.41. Calc. for $\mathrm{C}_{10} \mathrm{H}_{7} \mathrm{Br}_{2} \mathrm{NO}$ : C, 37.89; $\mathrm{H}, 2.23 ; \mathrm{N}, 4.42 \%$.

\section{2-(4-Biphenyl)-3,3-dibromoacrylonitrile (2l)}

Brown solid; ${ }^{1} \mathrm{H}$ NMR $\left(\mathrm{CDCl}_{3}, 400 \mathrm{MHz}\right): \delta 7.74-7.42$ (m, 9H, Ar-H); $\left.{ }^{13} \mathrm{C} \mathrm{NMR} \mathrm{(CDCl}, 100 \mathrm{MHz}\right): \delta$ 145.6, $139.1,132.5,129.0,128.6,127.9,127.7,127.2,122.1$, 118.9, 110.8; IR (KBr) $v_{\max } / \mathrm{cm}^{-1}: 3063,2922,2223,1600$, 1479, 1400, 842, 767. Found: C, 49.69; H, 2.49; N, 3.87. Calc. for $\mathrm{C}_{15} \mathrm{H}_{9} \mathrm{Br}_{2} \mathrm{~N}: \mathrm{C}, 49.62 ; \mathrm{H}, 2.50 ; \mathrm{N}, 3.86 \%$.

\section{1,3-bis(2,2-dibromo-1-cyanovinyl)benzene (2m)}

White solid; ${ }^{1} \mathrm{H}$ NMR $\left(\mathrm{CDCl}_{3}, 400 \mathrm{MHz}\right): \delta 7.75-7.42$ (m, 4H, Ar-H); ${ }^{13} \mathrm{C}$ NMR $\left(\mathrm{CDCl}_{3}, 100 \mathrm{MHz}\right): \delta$ 133.3, $132.4,132.3,132.2,131.5,128.5,128.4 ; \mathrm{IR}(\mathrm{KBr}) v_{\max } / \mathrm{cm}^{-1}$ : 3055, 2923, 2200, 1636, 1479, 1433, 1102, 713, 514. Found: C, 29.14; $\mathrm{H}, 0.81 ; \mathrm{N}, 5.63$. Calc. for $\mathrm{C}_{12} \mathrm{H}_{4} \mathrm{Br}_{4} \mathrm{~N}_{2}$ : C, $29.07 ; \mathrm{H}, 0.81 ; \mathrm{N}, 5.65 \%$.

\section{2-(Furan-2-yl)-3,3-dibromoacrylonitrile (2n)}

White solid; ${ }^{1} \mathrm{H}$ NMR $\left(\mathrm{CDCl}_{3}, 400 \mathrm{MHz}\right): \delta 7.58(\mathrm{~d}$, $1 \mathrm{H}, J 1.6 \mathrm{~Hz}, \mathrm{Fu}-\mathrm{H}$ ), 7.08 (d, 1H, $J 4.0 \mathrm{~Hz}, \mathrm{Fu}-\mathrm{H}), 6.54$ $(\mathrm{dd}, 1 \mathrm{H}, J 3.6 \mathrm{~Hz}, J 1.6 \mathrm{~Hz}, \mathrm{Fu}-\mathrm{H}),{ }^{13} \mathrm{C}$ NMR $\left(\mathrm{CDCl}_{3}, 100\right.$ $\mathrm{MHz}): \delta 145.9,144.2,115.3,114.5,113.5,112.1,103.7$. IR $(\mathrm{KBr}) v_{\max } / \mathrm{cm}^{-1}: 3153,2227,1476,1030,850,750$. Found: $\mathrm{C}, 30.29 ; \mathrm{H}, 1.08 ; \mathrm{N}, 5.08$. Calc. for $\mathrm{C}_{7} \mathrm{H}_{3} \mathrm{Br}_{2} \mathrm{NO}: \mathrm{C}, 30.36$; $\mathrm{H}, 1.09$; N, $5.06 \%$.

\section{Supplementary Information}

Full set of IR, ${ }^{1} \mathrm{H}$ NMR and ${ }^{13} \mathrm{C}$ NMR spectra are available free of charge at http://jbcs.sbq.org.br, as pdf file.

\section{Acknowledgments}

The authors thank the National Natural Science Foundation of China (20772096), and Key Laboratory of Eco-Environment-Related Polymer Materials (Northwest Normal University), Ministry of Education of China for the financial support of this work.

\section{References}

1. Shablykin, O. V.; Gakh, A. A.; Brovarets, V. S.; Rusanov, E. B.; Drach, B. S.; Heteroat. Chem. 2008, 9, 506; Shablykin, O. V.; Brovarets, V. S.; Drach, B. S.; Russ. J. Gen. Chem. 2007, 77, 1308; Brovarets, V. S.; Pilyo, S. G.; Chernega, A. N.; Romanenko, E. A.; Drach, B. S.; Russ. J. Gen. Chem. 1999, 69, 1577; Popil'nichenko, S. V.; Brovarets, V. S.; Chernega, A. N.; Poltorak, D. V.; Drach, B. S.; Heteroat. Chem. 2006, 17, 411; Golovchenko, O. V.; Pilyo, S. G.; Brovarets, V. S.; Chernega, A. N.; Drach, B. S.; Heteroat. Chem. 2004, 15, 454; Pilyo, S. G.; Brovarets, V. S.; Vinogradova, T. K.; Golovchenko, A. V.; Drach, B. S.; Russ. J. Gen. Chem. 2002, 72, 1714; Kozachenko, A. P.; Shablykin, O. V.; Vasilenko, A. N.; Brovarets, V. S.; Russ. J. Gen. Chem. 2010, 80, 127; Kozachenko, A. P.; Shablykin, O. V.; Rusanov, E. B.; Vasilenko, A. N.; Brovarets, V. S.; Russ. J. Gen. Chem. 2009, 79, 996.

2. Soulen, R. L.; Carlson, S. C.; Lang, F.; J. Org. Chem. 1973, 38, 479; Clement, B. A.; Soulen, R. L.; J. Org. Chem. 1974, 39, 97; Clement, B. A.; Soulen, R. L.; J. Org. Chem. 1976, 41, 556; Sepiol, J. J.; Sepiol, J. A.; Soulen, R. L.; J. Org. Chem. 1984, 49, 1125.

3. Haase, K.; Hoffmann, H. M.; Angew. Chem., Int. Ed. 1982, 21, 83.

4. Kazuaki, S.; Bull. Chem. Soc. Jpn. 1987, 60, 1085.

5. Oakwood, T. S.; Weisgerber, C. A.; Org. Synth. 1955, 24, 14.

6. Patricinio, A. F.; Moran, P. J. S.; J. Braz. Chem. Soc. 2001, 12, 7; Cao, Y. Q.; Du, Y. F.; Chen, B. H.; Li, J. T.; Synth. Commum. 2004, 34, 2951.

7. Harle, H.; Jochims, J. C.; Chem. Ber. 1986, 119, 1400; Zeng, W.; Yang, J.; Meng, B.; Zhang, B.; Jiang, M.; Chen, F. X.; Lett. Org. Chem. 2009, 6, 637.

8. Schareina, T.; Zapf, A.; Beller, M.; J. Organomet. Chem. 2004, 689, 4576; Schareina, T.; Zapf, A.; Beller, M.; Chem. Commun. 2004, 1388; Schareina, T.; Zapf, A.; Beller, M.; Tetrahedron Lett. 2005, 46, 2585; Schareina, T.; Zapf, A.; Magerlein, W.; Muller, N.; Beller, M.; Tetrahedron Lett. 2007, 48, 1087; Weissman, S. A.; Zewge, D.; Chen, C.; J. Org. Chem. 2005, 70, 1508; Grossman, 
O.; Gelman, D.; Org. Lett. 2006, 8, 1189; Li, L. H.; Pan, Z. L.; Duan, X. H.; Liang, Y. M.; Synlett 2006, 2094; Velmathi, S.; Leadbeater, N. E.; Tetrahedron Lett. 2008, 49, 4693; Chen, G.; Weng, J.; Zheng, Z.; Zhu, X.; Cai, Y.; Cai, J.; Wan, Y.; Eur. J. Org. Chem. 2008, 3524; Cheng, Y. N.; Duan, Z.; Yu, L. J.; Li, Z. X.; Zhu, Y.; Wu, Y. J.; Org. Lett. 2008, 10, 901; Franz, A. W.; Popa, L. N.; Muller, T. J. J.; Tetrahedron Lett. 2008, 49, 3300; Ren, Y.
L.; Liu, Z. F.; He, S. B.; Zhao, S.; Wang, J. J.; Niu, R. Q.; Yin, W. P.; Org. Process Res. Dev. 2009, 13, 764.

9. Li, Z.; Shi, S. Y.; Yang, J. Y.; Synlett 2006, 2495.

Submitted: April 27, 2010

Published online: September 8, 2010 


\section{Supplementary Information}

\section{One-Pot Four-Component Synthesis of 2-Aryl-3,3-Dihaloacrylonitriles using Potassium Hexacyanoferrate(II) as Environmentally Benign Cyanide Source}

\section{Zhouxing Zhao and Zheng Li*}

Key Laboratory of Polymer Materials of Gansu Province, College of Chemistry and Chemical Engineering, Northwest Normal University, Lanzhou, Gansu, 730070, P. R. China

The IR, ${ }^{1} \mathrm{H}$ MNR and ${ }^{13} \mathrm{C}$ MNR spectra for products are given below:

2-Phenyl-3,3-dichloroacrylonitrile (1a)

IR (KBr) $v_{\max } / \mathrm{cm}^{-1}: 3061,2922,2223,1563,1445,930,824,759,695 ;{ }^{1} \mathrm{H}$ NMR $\left(\mathrm{CDCl}_{3}, 400 \mathrm{MHz}\right): \delta$ 7.52-7.41 (m, $5 \mathrm{H}, \mathrm{Ph}-\mathrm{H}),{ }^{13} \mathrm{C} \mathrm{NMR}\left(\mathrm{CDCl}_{3}, 100 \mathrm{MHz}\right): \delta 137.1,130.6,130.0,128.8,128.6,115.5,108.2$.

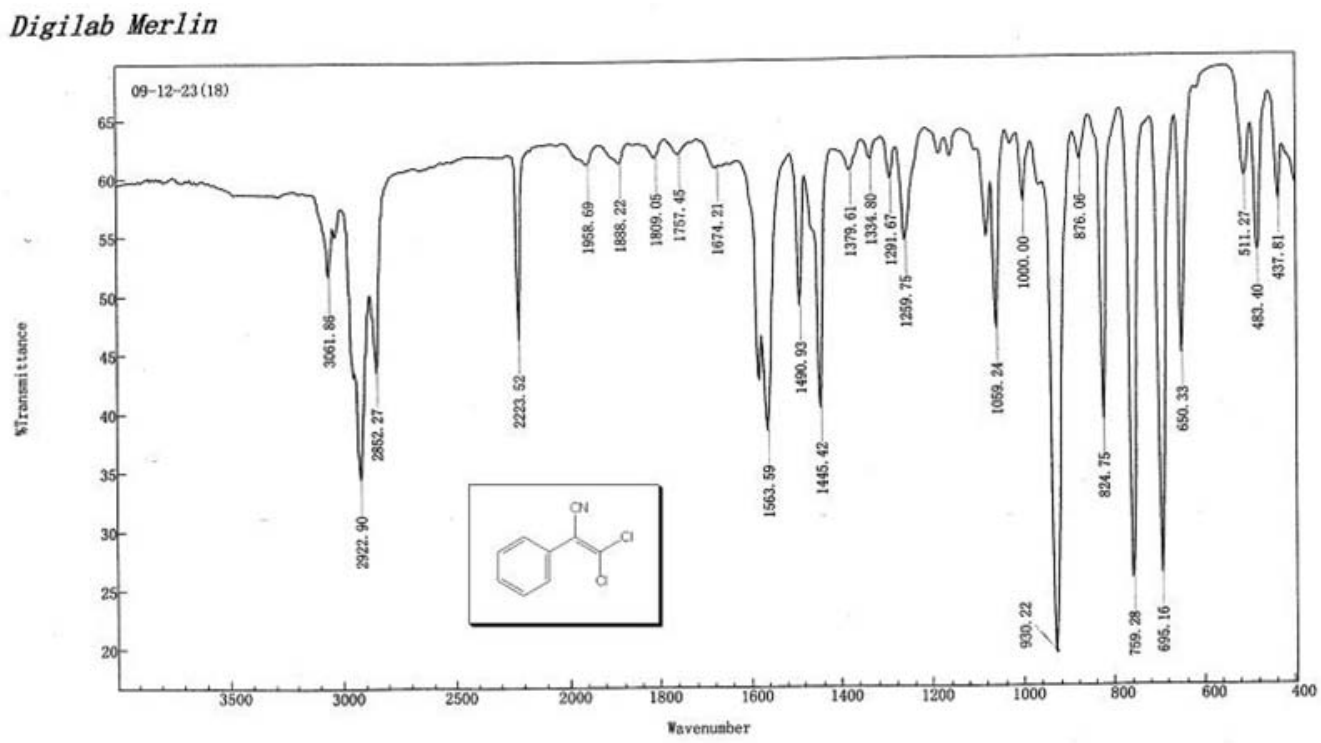

Figure S1. IR spectrum of 2-phenyl-3,3-dichloroacrylonitrile (1a). 


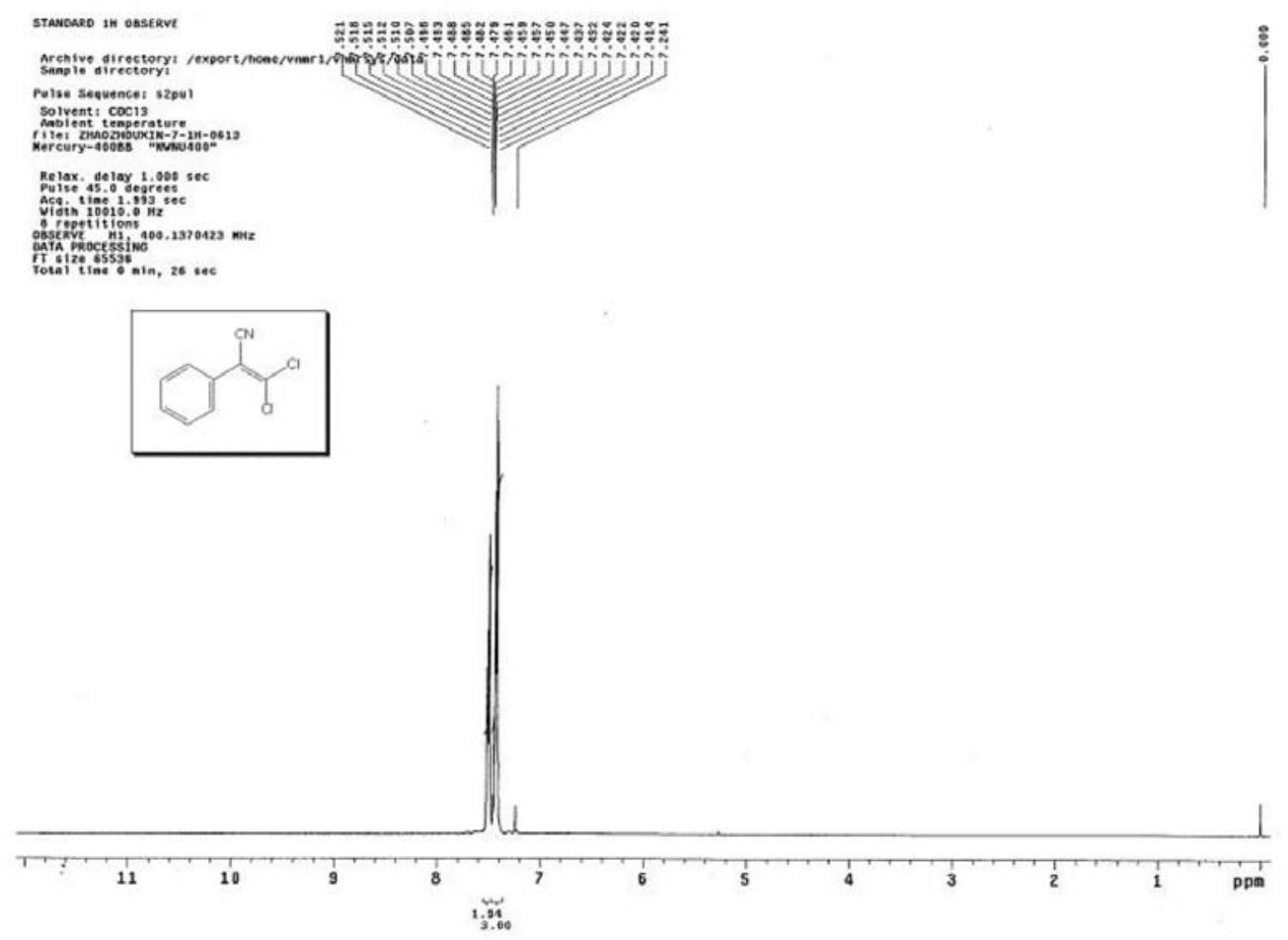

Figure S2 'H NMR spectrum of 2-phenyl-3,3-dichloroacrylonitrile (1a)
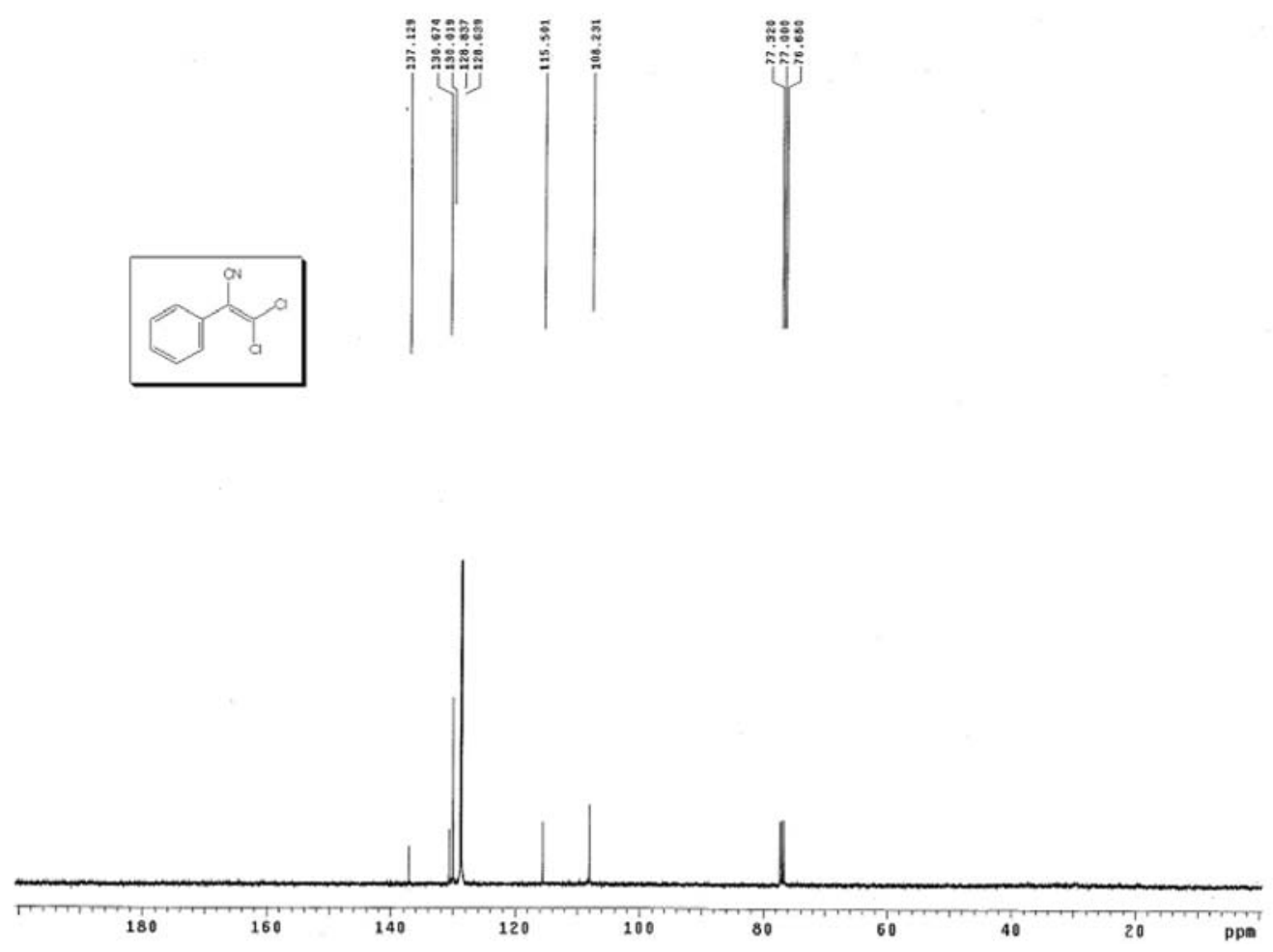

Figure S3. ${ }^{13} \mathrm{C}$ NMR spectrum of 2-phenyl-3,3-dichloroacrylonitrile (1a). 
2-(2-Cholorophenyl)-3,3-dichloroacrylonitrile (1b)

IR (KBr) $v_{\max } / \mathrm{cm}^{-1}: 3042,2999,2219,1604,1510,1256.927,825 ;{ }^{1} \mathrm{H}$ NMR (CDCl, $\left.400 \mathrm{MHz}\right): \delta 7.60-7.31(\mathrm{~m}, 4 \mathrm{H}$, $\mathrm{Ph}-\mathrm{H}),{ }^{13} \mathrm{C}$ NMR $\left(\mathrm{CDCl}_{3}, 100 \mathrm{MHz}\right): \delta 132.9,132.4,131.5,130.4,130.2,127.4,120.4,115.6,113.9$.

Digilab Merlin

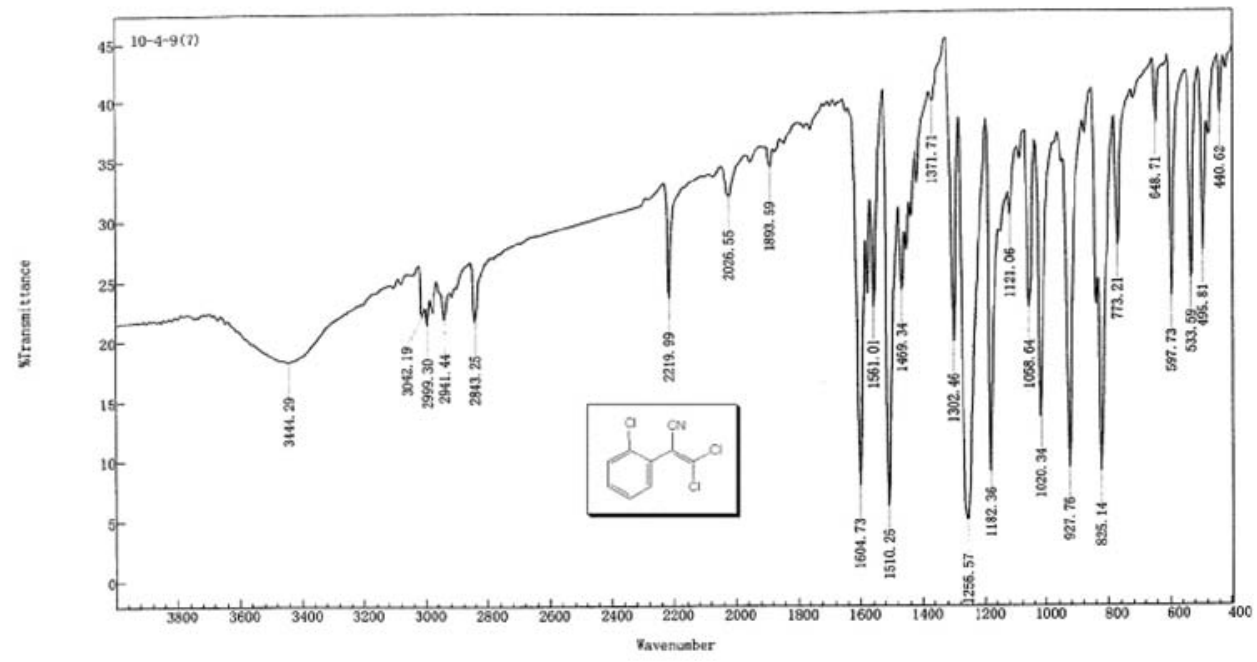

Figure S4. IR spectrum of 2-(2-cholorophenyl)-3,3-dichloroacrylonitrile (1b).

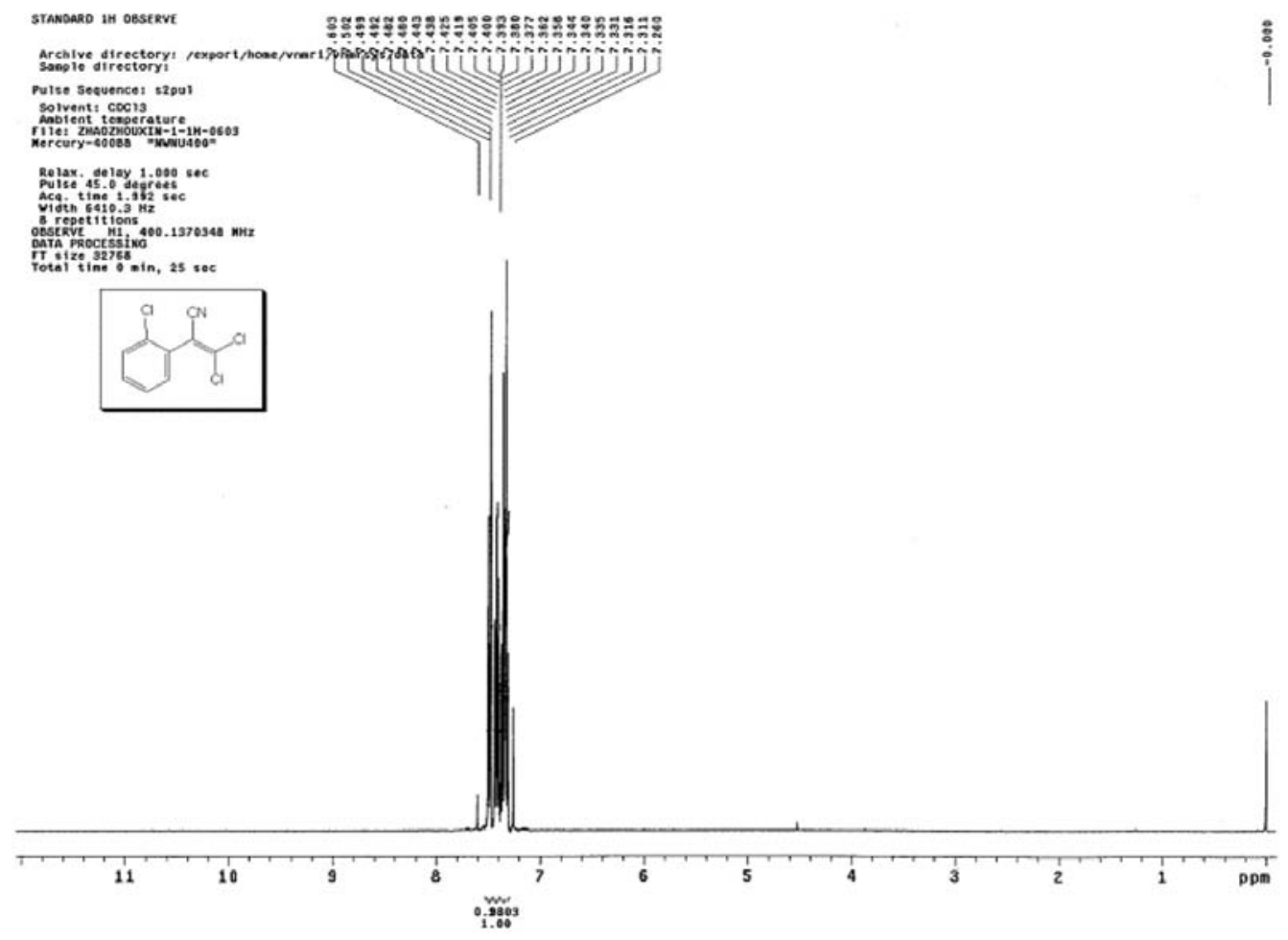

Figure S5. ${ }^{1} \mathrm{H}$ NMR spectrum of 2-(2-cholorophenyl)-3,3-dichloroacrylonitrile (1b). 

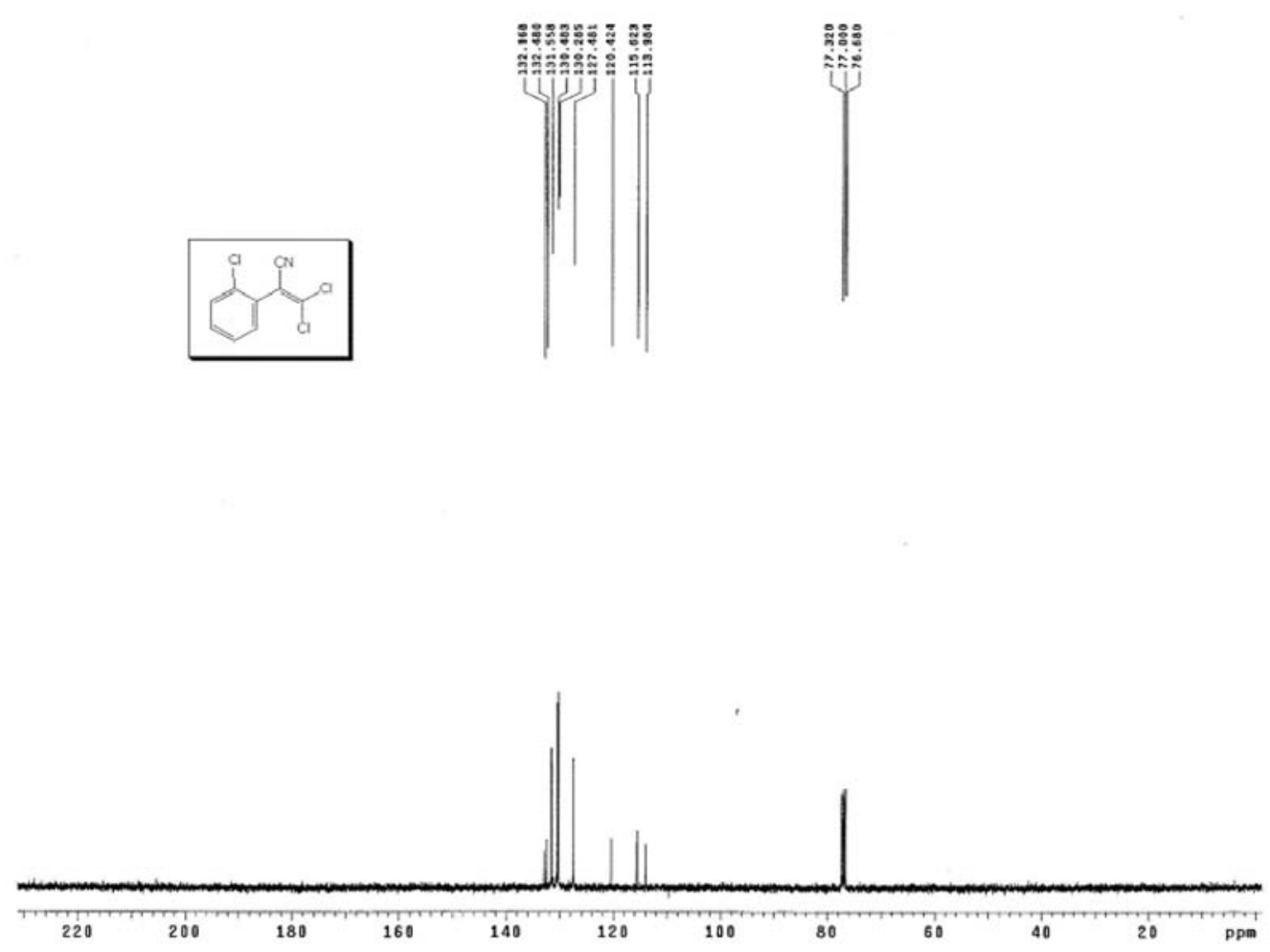

Figure S6. ${ }^{13} \mathrm{C}$ NMR spectrum of 2-(2-cholorophenyl)-3,3-dichloroacrylonitrile (1b).

\section{2-(3-Cholorophenyl)-3,3-dichloroacrylonitrile (1c)}

IR $(\mathrm{KBr}) v_{\max } / \mathrm{cm}^{-1}: 3062,2926,2220,1571,1471,1262.943,850 ;{ }^{1} \mathrm{H}$ NMR $\left(\mathrm{CDCl}_{3}, 400 \mathrm{MHz}\right): \delta 7.52(\mathrm{~s}, 1 \mathrm{H}, \mathrm{Ph}-\mathrm{H})$, 7.44-7.39 (m, 3H, Ph-H); ${ }^{13} \mathrm{C}$ NMR $\left(\mathrm{CDCl}_{3}, 100 \mathrm{MHz}\right): \delta$ 138.6, 134.9, 132.2, 130.3, 130.2, 128.8, 126.9, 115.1, 114.3.

Digilab Merlin

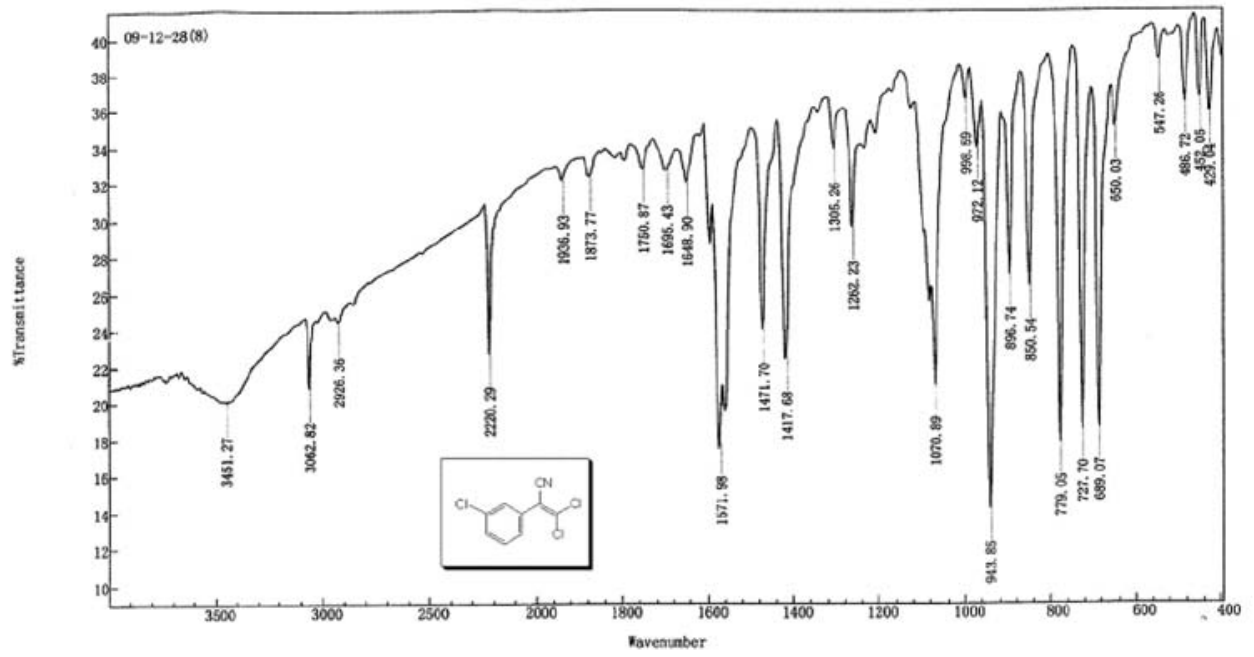

Figure S7. IR spectrum of 2-(3-cholorophenyl)-3,3-dichloroacrylonitrile (1c). 


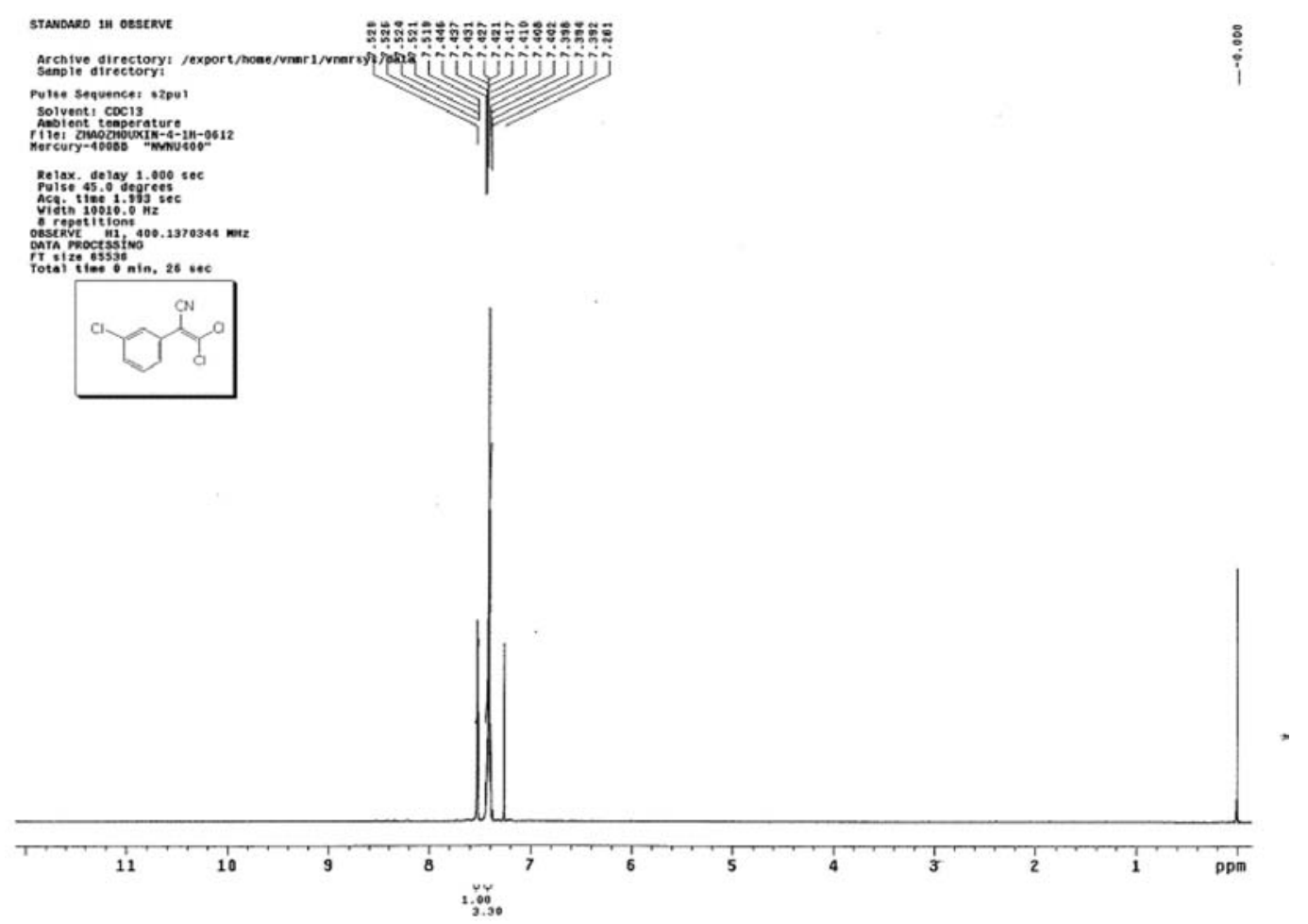

Figure S8. ${ }^{1} \mathrm{H}$ NMR spectrum of 2-(3-cholorophenyl)-3,3-dichloroacrylonitrile (1c).
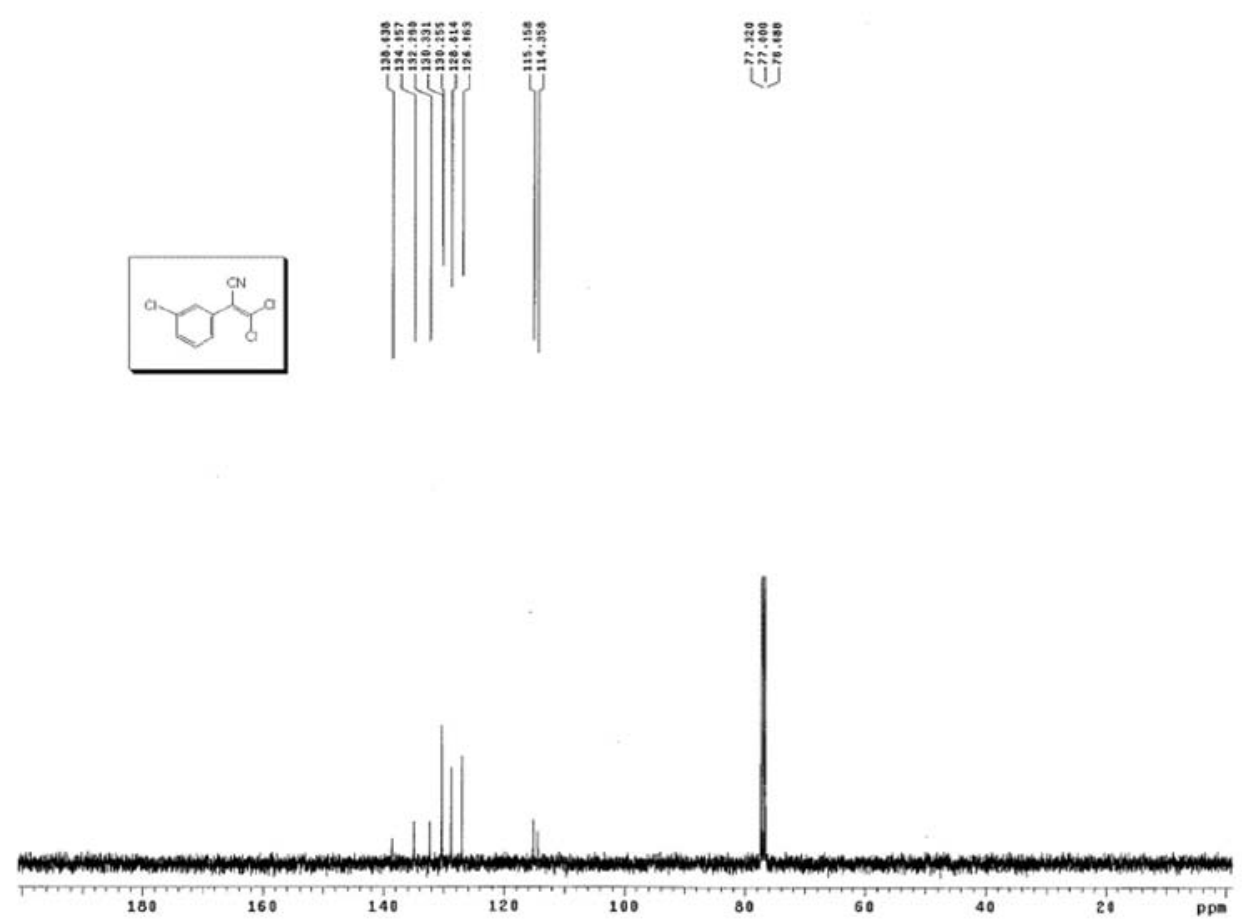

Figure S9. ${ }^{13} \mathrm{C}$ NMR spectrum of 2-(3-cholorophenyl)-3,3-dichloroacrylonitrile (1c). 


\section{2-(4-Cholorophenyl)-3,3-dichloroacrylonitrile (1d)}

IR (KBr) $v_{\max } / \mathrm{cm}^{-1}: 3084,2924,2220,1555,1481,1279.927,809 ;{ }^{1} \mathrm{H}$ NMR $\left(\mathrm{CDCl}_{3}, 400 \mathrm{MHz}\right): \delta$ 7.48-7.40 (m, 4H, $\mathrm{Ph}-\mathrm{H}) ;{ }^{13} \mathrm{C} \mathrm{NMR}\left(\mathrm{CDCl}_{3}, 100 \mathrm{MHz}\right): \delta$ 137.7, 136.2, 130.0, 129.1, 129.0, 115.2, 114.5 .

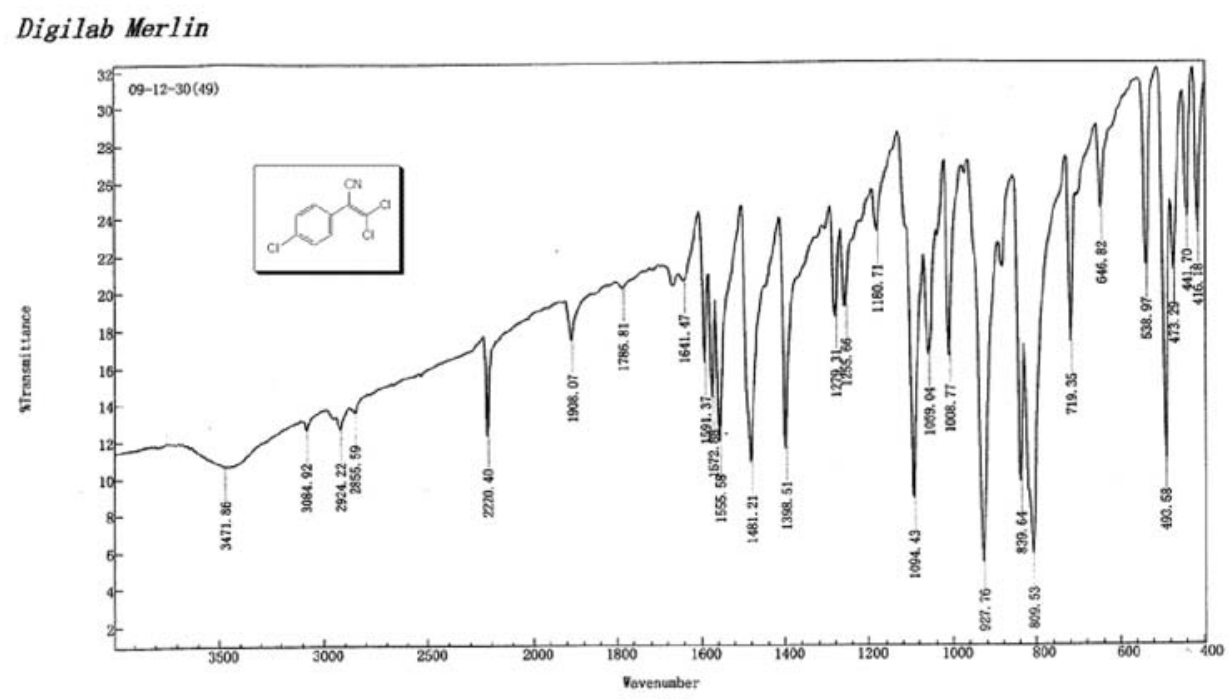

Figure S10. IR spectrum of 2-(4-cholorophenyl)-3,3-dichloroacrylonitrile (1d).

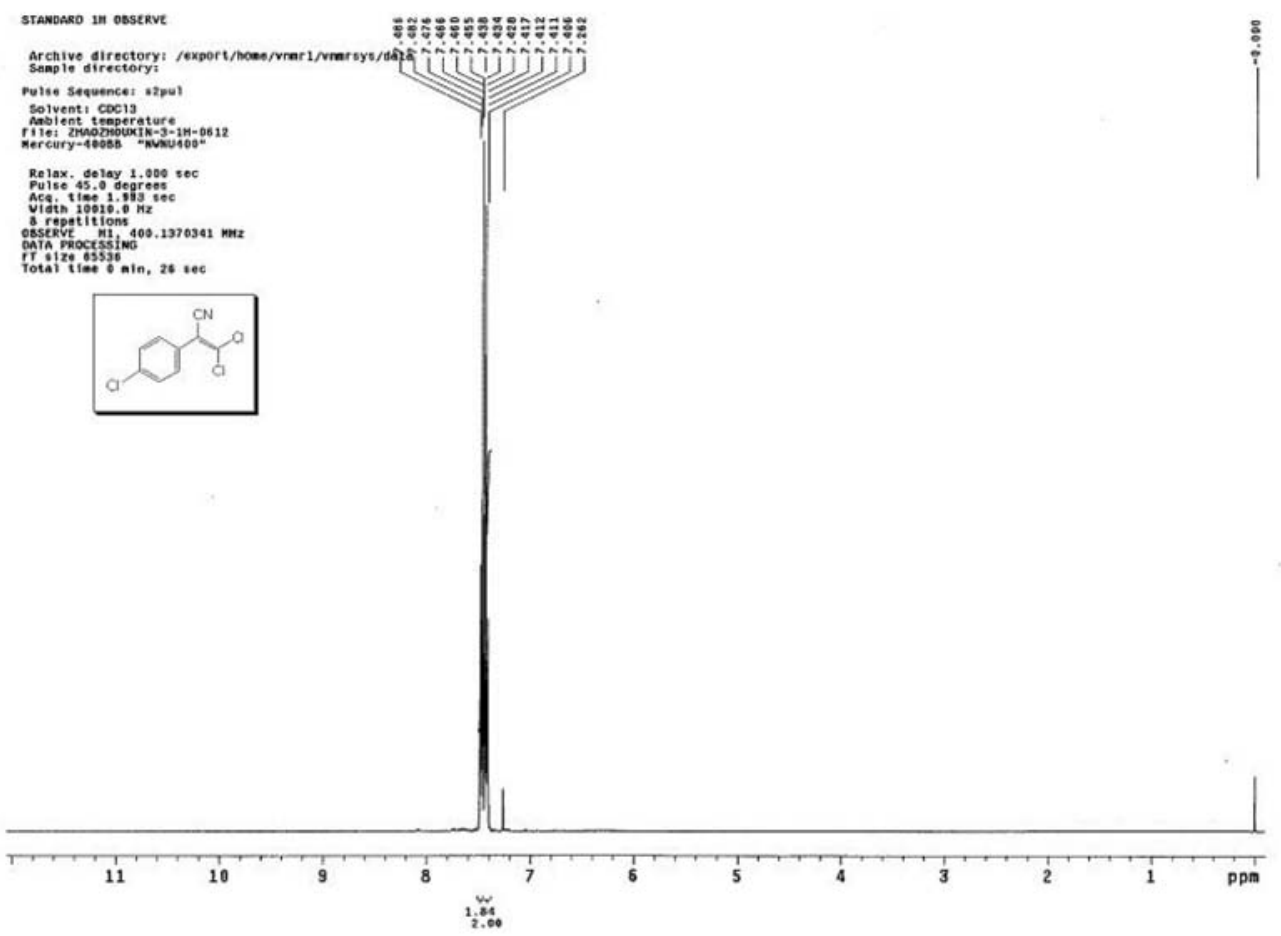

Figure S11. ${ }^{1} \mathrm{H}$ NMR spectrum of 2-(4-cholorophenyl)-3,3-dichloroacrylonitrile (1d). 


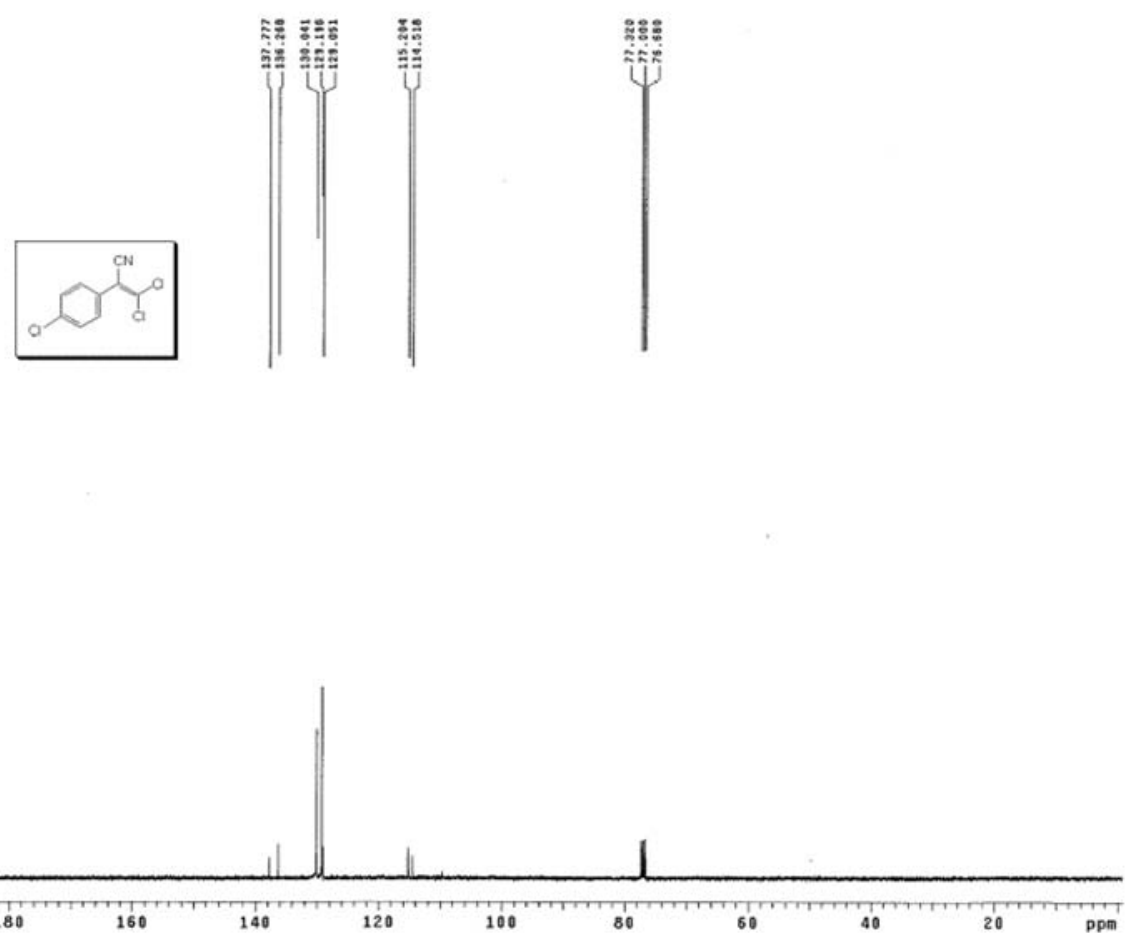

Figure S12. ${ }^{13} \mathrm{C}$ NMR spectrum of 2-(4-cholorophenyl)-3,3-dichloroacrylonitrile (1d).

\section{2-(2-Methylphenyl)-3,3-dichloroacrylonitrile (1e)}

IR (KBr) $v_{\max } / \mathrm{cm}^{-1}: 3067,2925,2219,1571,1485,1258,930,753 ;{ }^{1} \mathrm{H}$ NMR $\left(\mathrm{CDCl}_{3}, 400 \mathrm{MHz}\right): \delta$ 7.22-7.00 (m, 4H, $\mathrm{Ph}-\mathrm{H}), 2.16$ (s, $\left.3 \mathrm{H}, \mathrm{CH}_{3}\right) ;{ }^{13} \mathrm{CNMR}\left(\mathrm{CDCl}_{3}, 100 \mathrm{MHz}\right): \delta 138.5,136.1,130.4,130.0,129.9,128.7,126.2,114.7,114.2,18.8$.

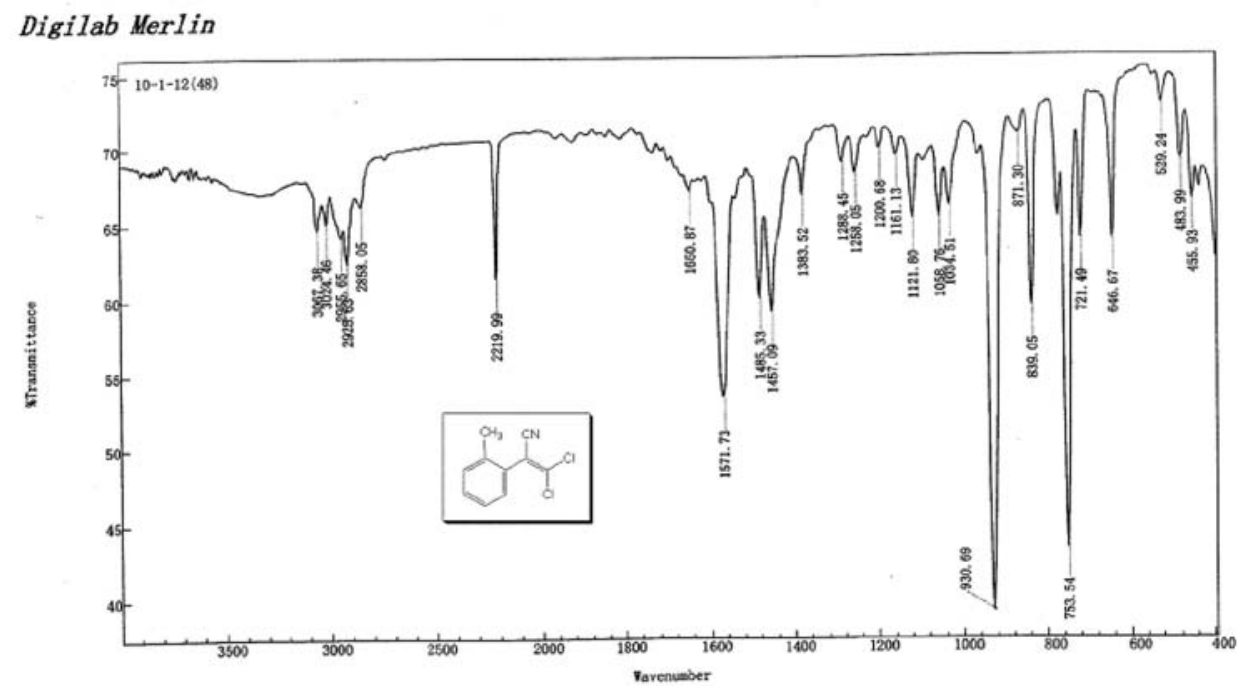

Figure S13. IR spectrum of 2-(2-methylphenyl)-3,3-dichloroacrylonitrile (1e). 


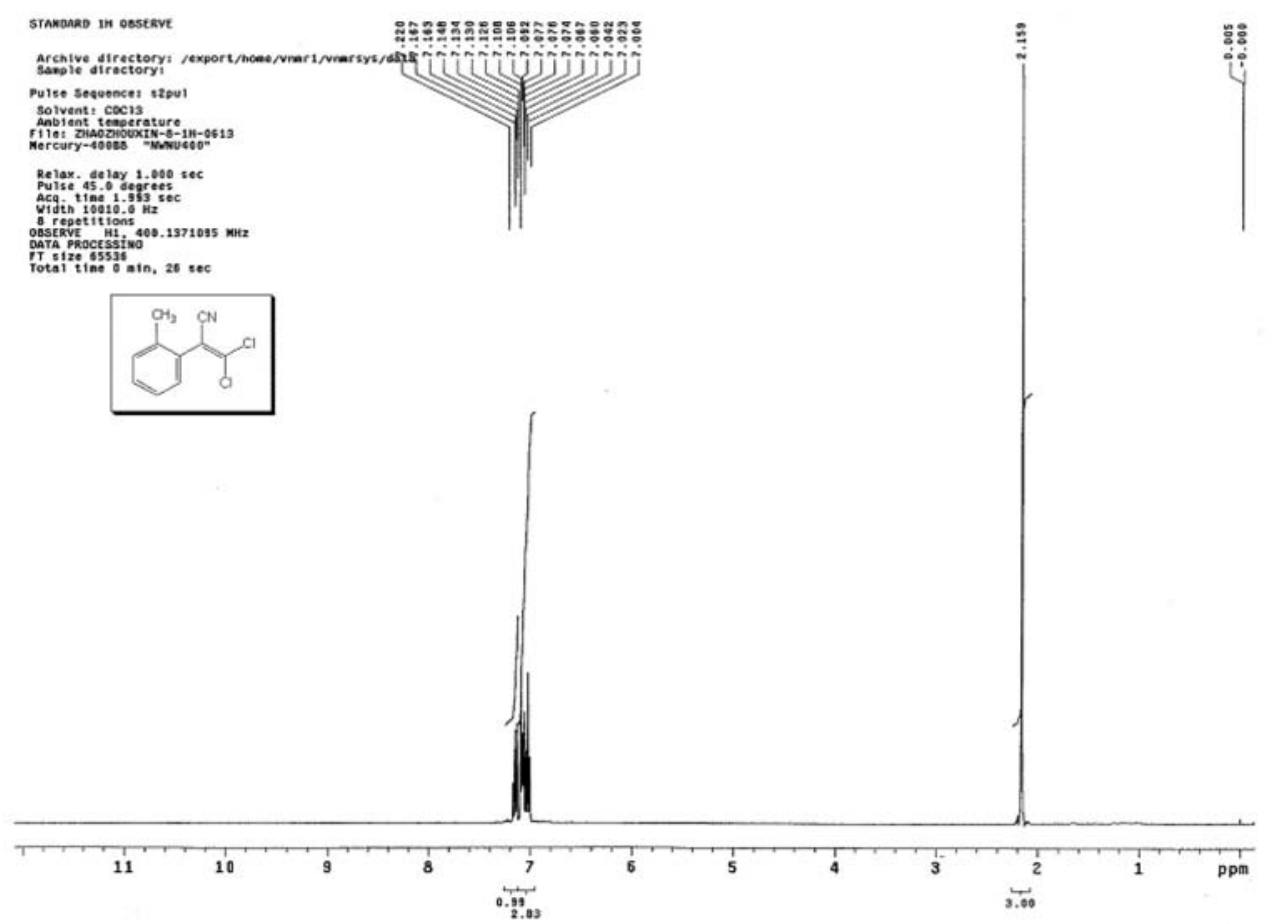

Figure S14. ${ }^{1} \mathrm{H}$ NMR spectrum of 2-(2-methylphenyl)-3,3-dichloroacrylonitrile (1e).

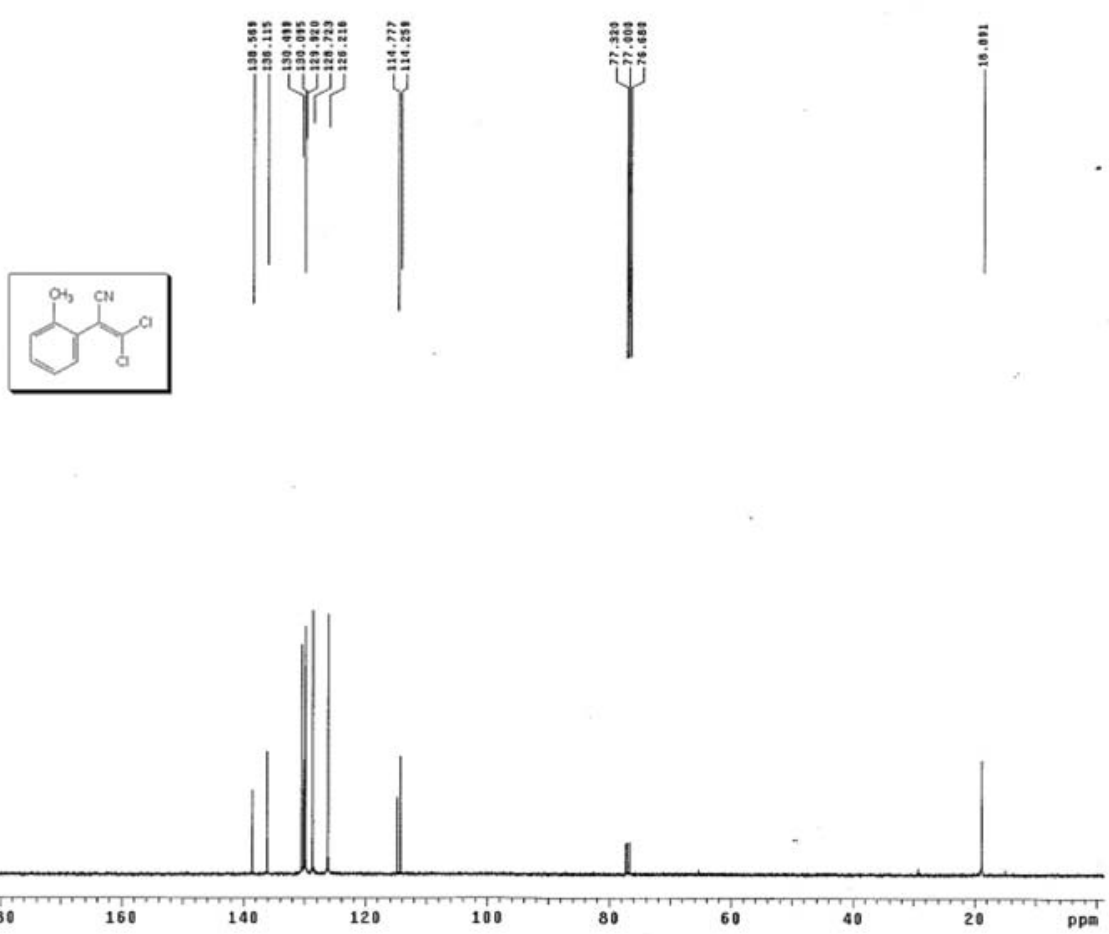

Figure S14. ${ }^{13} \mathrm{C}$ NMR spectrum of 2-(2-methylphenyl)-3,3-dichloroacrylonitrile (1e). 
2-(3-Methylphenyl)-3,3-dichloroacrylonitrile (1f)

IR (KBr) $v_{\max } / \mathrm{cm}^{-1}: 3039,2923,2222,1565,1485,1267,931,877,791,697 ;{ }^{1} \mathrm{H}$ NMR $\left(\mathrm{CDCl}_{3}, 400 \mathrm{MHz}\right): \delta 7.28-7.20$ (m, 4H, Ar-H), 2.35 (s, 3H, $\left.\mathrm{CH}_{3}\right) ;{ }^{13} \mathrm{C}$ NMR $\left(\mathrm{CDCl}_{3}, 100 \mathrm{MHz}\right): \delta$ 138.5, 136.6, 130.6, 130.4, 128.9, 128.5, 125.5, 115.5, 115.4, 21.0.

\section{Digilab Merlin}

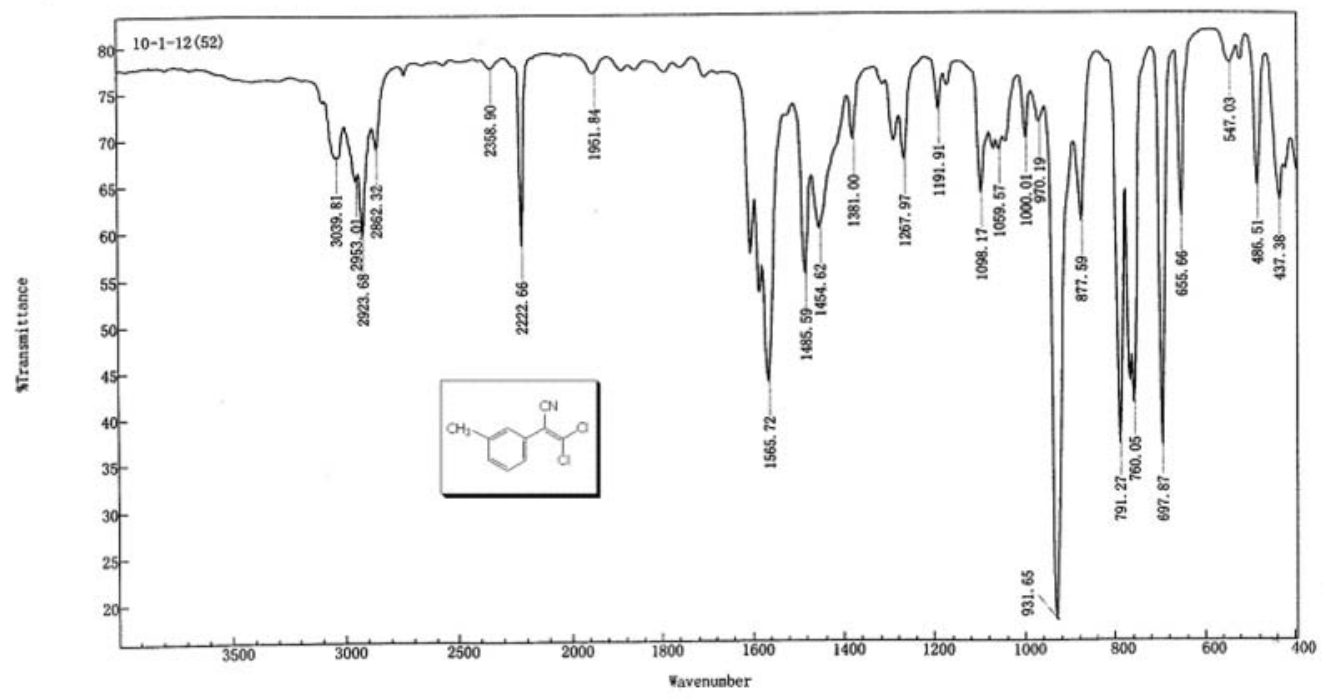

Figure S16. IR spectrum of 2-(3-methylphenyl)-3,3-dichloroacrylonitrile (1f).

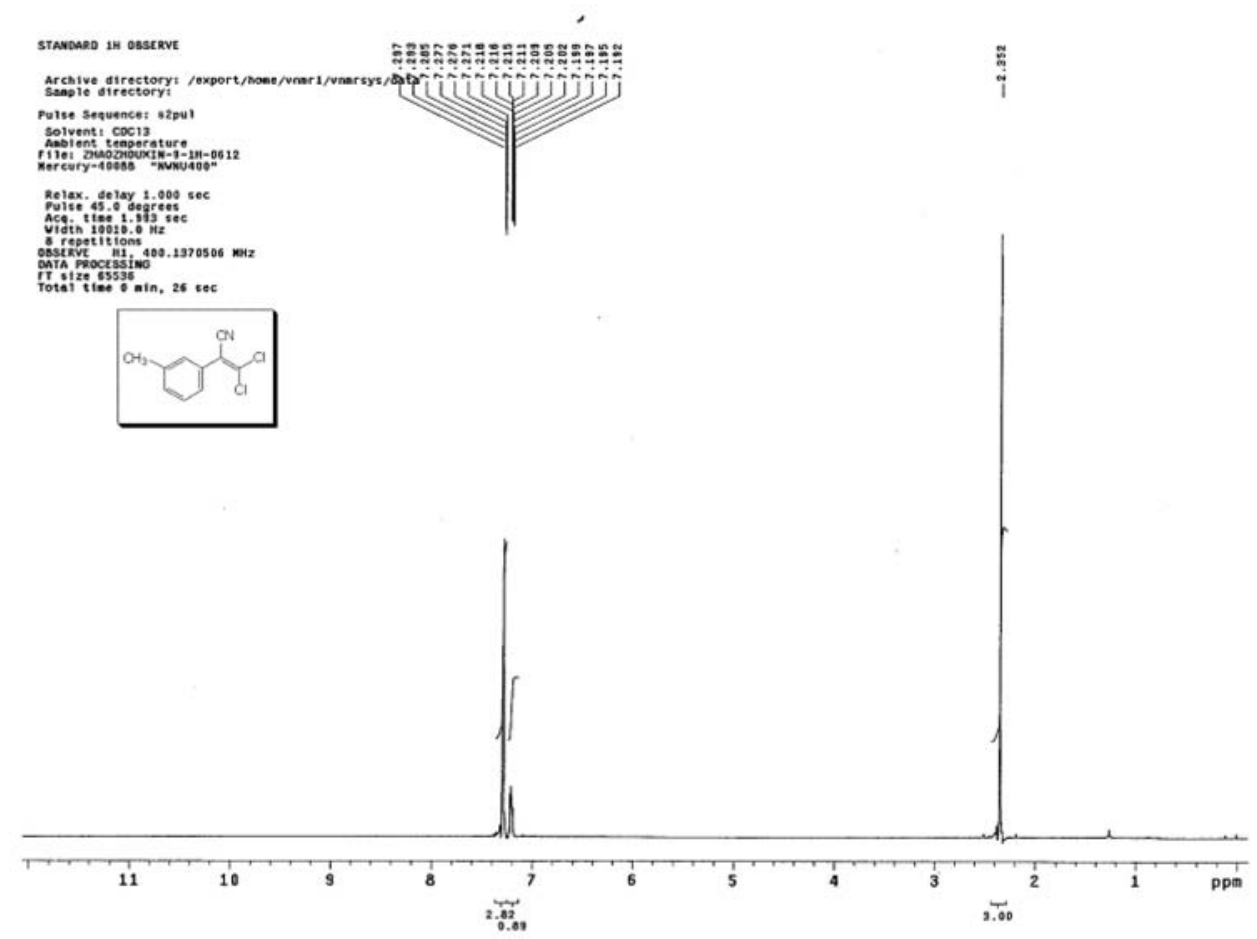

Figure S17. ${ }^{1} \mathrm{H}$ NMR spectrum of 2-(3-methylphenyl)-3,3-dichloroacrylonitrile (1f). 


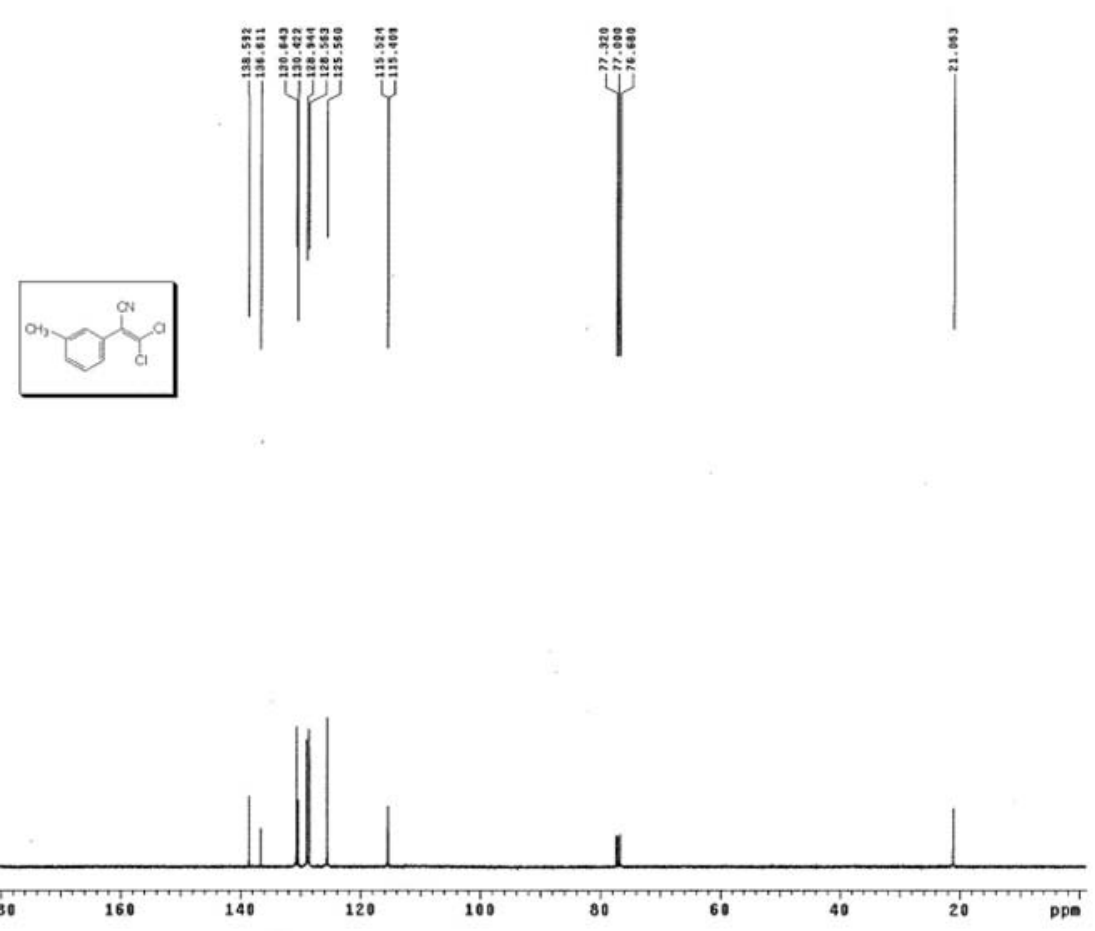

Figure S18. ${ }^{13} \mathrm{C}$ NMR spectrum of 2-(3-methylphenyl)-3,3-dichloroacrylonitrile (1f).

2-(4-Methylphenyl)-3,3- dichloroacrylonitrile (1g)

IR (KBr) $v_{\max } / \mathrm{cm}^{-1}: 3031,2923,2222,1573,1509,1258,929,815,771 ;{ }^{1} \mathrm{H}$ NMR $\left(\mathrm{CDCl}_{3}, 400 \mathrm{MHz}\right): \delta 7.37$ (d, $2 \mathrm{H}, J$ $8.4 \mathrm{~Hz}, \mathrm{Ar}-\mathrm{H}), 7.19$ (d, $2 \mathrm{H}, J$ 8.4 Hz, Ar-H), $2.32\left(\mathrm{~s}, 3 \mathrm{H}, \mathrm{CH}_{3}\right) ;{ }^{13} \mathrm{C} \mathrm{NMR}\left(\mathrm{CDCl}_{3}, 100 \mathrm{MHz}\right): \delta 140.0,135.7,129.2,128.2$, 127.4, 115.3, 115.2, 20.9 .

Digilab Merlin

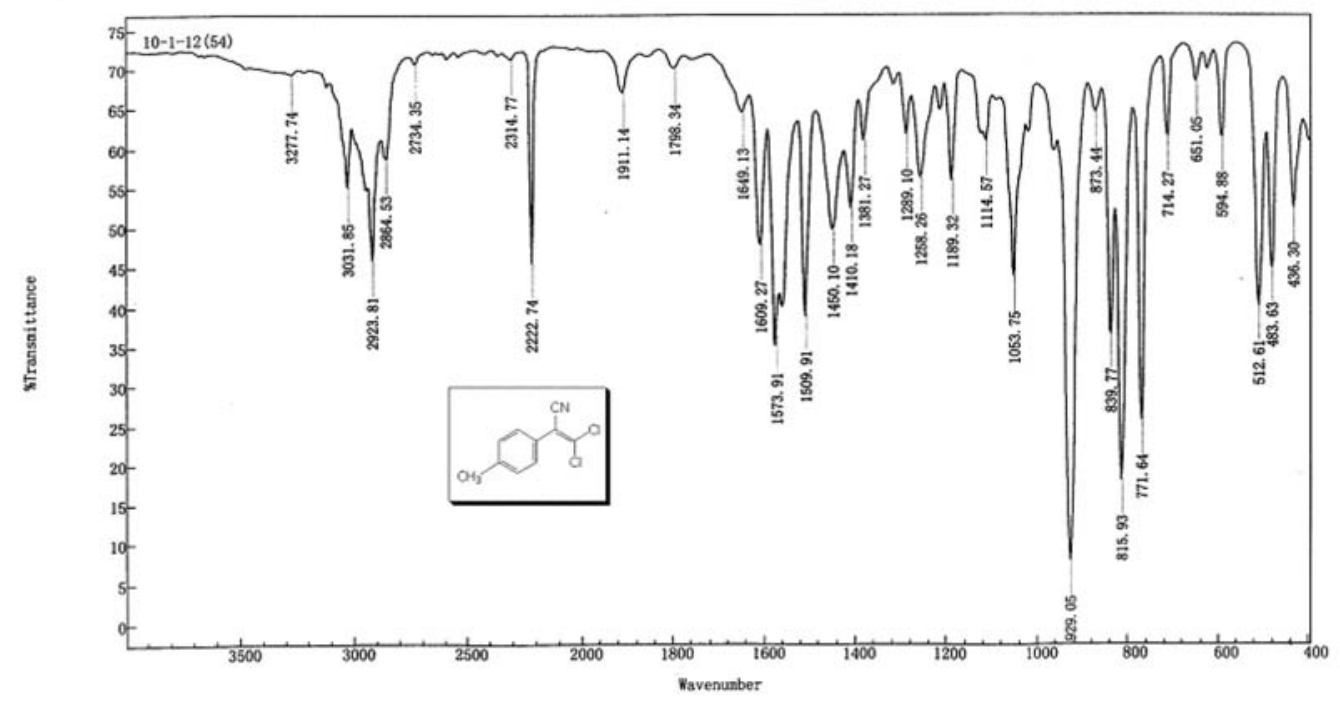

Figure S19. IR spectrum of 2-(4-methylphenyl)-3,3-dichloroacrylonitrile (1g). 


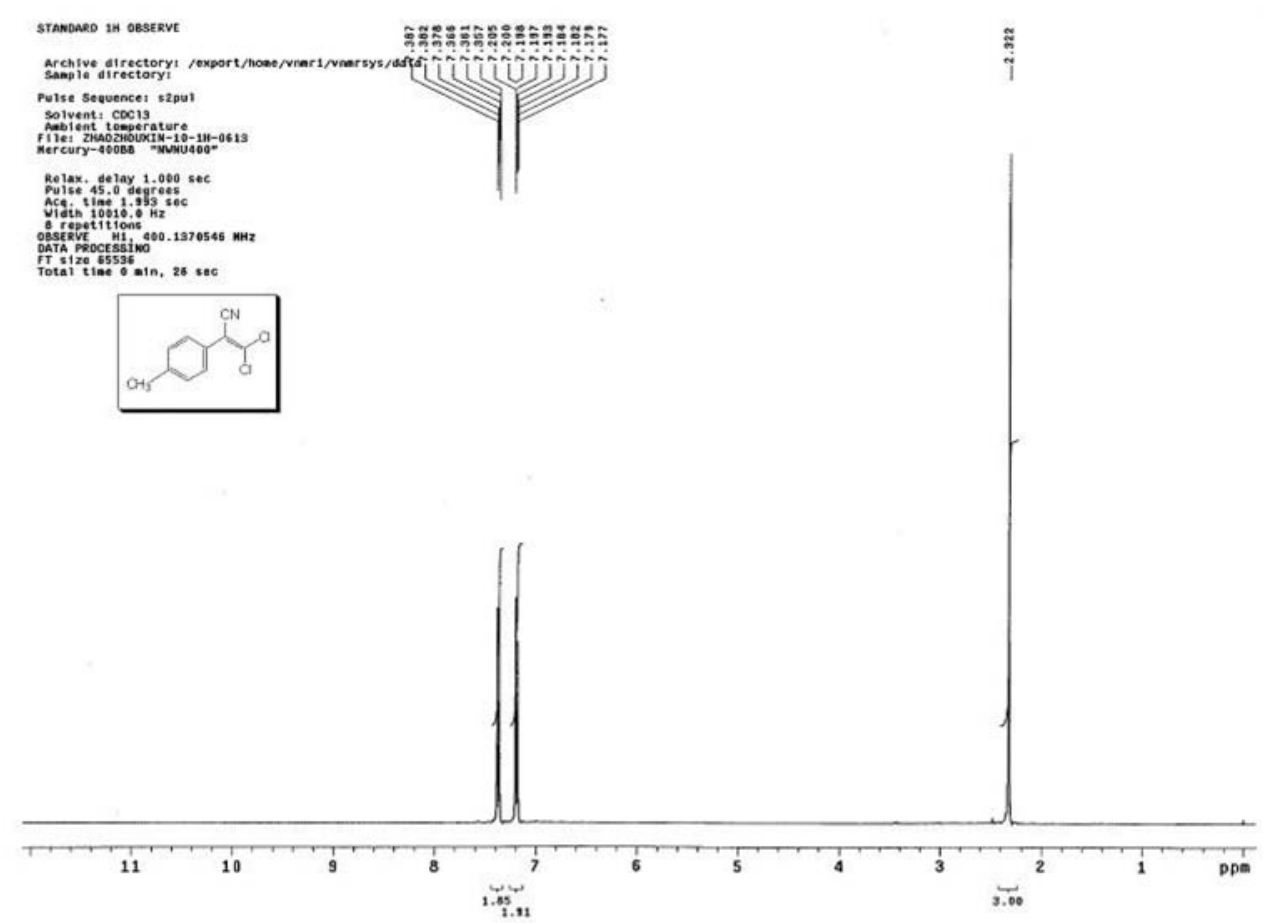

Figure S20. 'H NMR spectrum of 2-(4-methylphenyl)-3,3-dichloroacrylonitrile (1g).

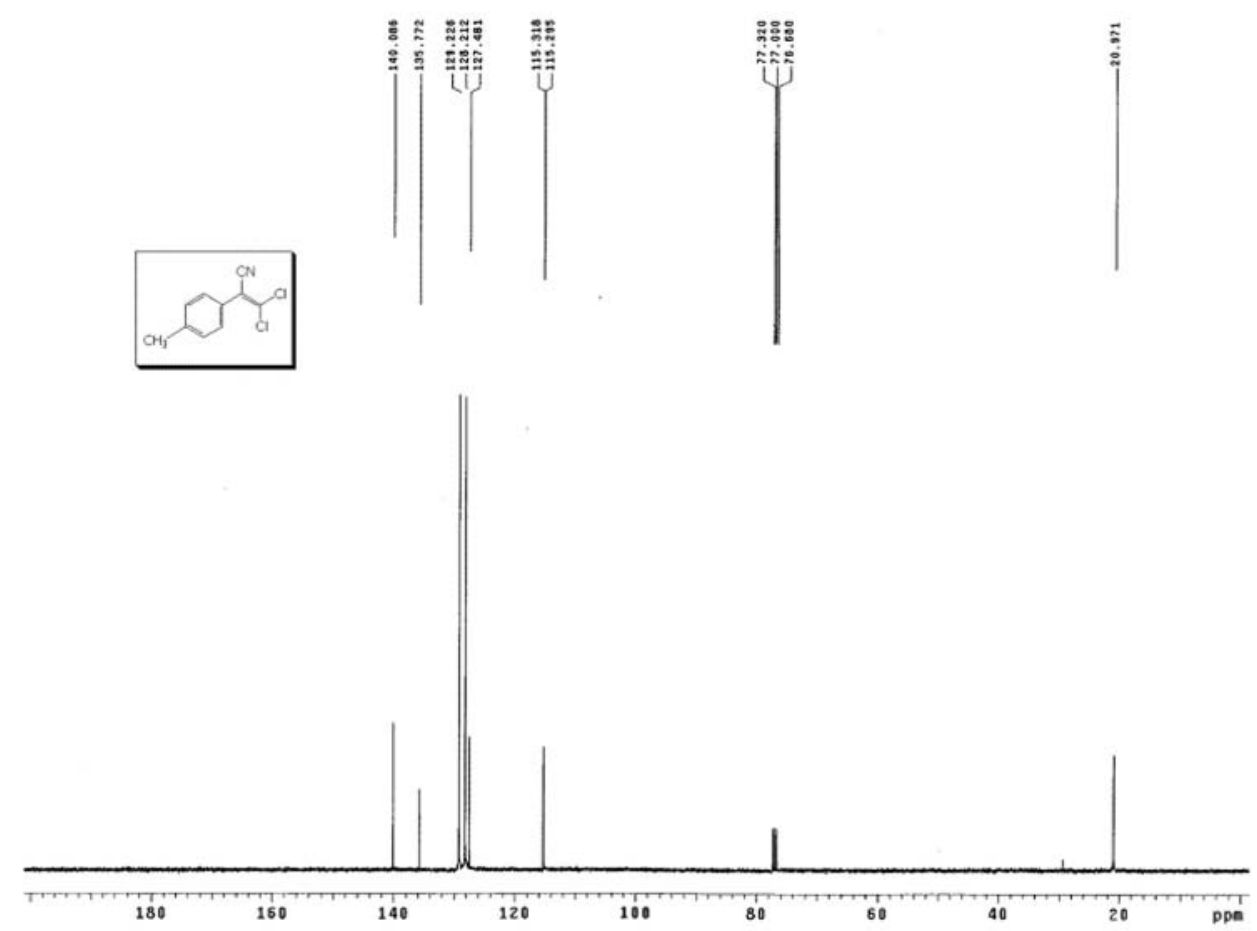

Figure S21. ${ }^{13} \mathrm{C}$ NMRspectrum of 2-(4-methylphenyl)-3,3-dichloroacrylonitrile (1g). 


\section{2-(2-Ethylphenyl)-3,3- dichloroacrylonitrile (1h)}

IR (KBr) $v_{\max } / \mathrm{cm}^{-1}: 3032,2918,2224,1589,1497,1234,942,842 ;{ }^{1} \mathrm{H} \mathrm{NMR}\left(\mathrm{CDCl}_{3}, 400 \mathrm{MHz}\right): \delta 7.25-6.97$ (m, 4H,

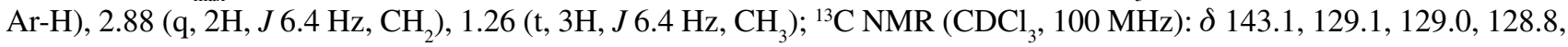
124.7, 124.1, 121.6, 120.3, 119.2, 25.6, 14.3.

\section{Digilab Merlin}

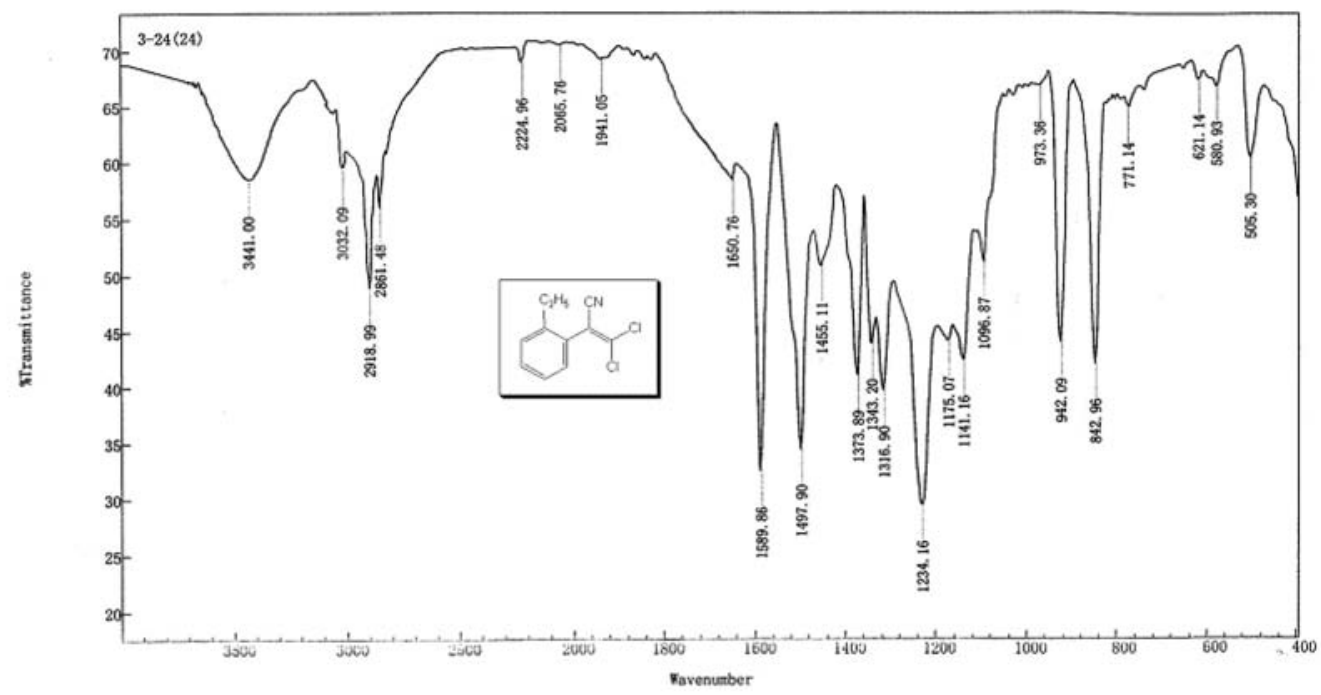

Figure S22. IR spectrum of 2-(2-ethylphenyl)-3,3-dichloroacrylonitrile (1h).

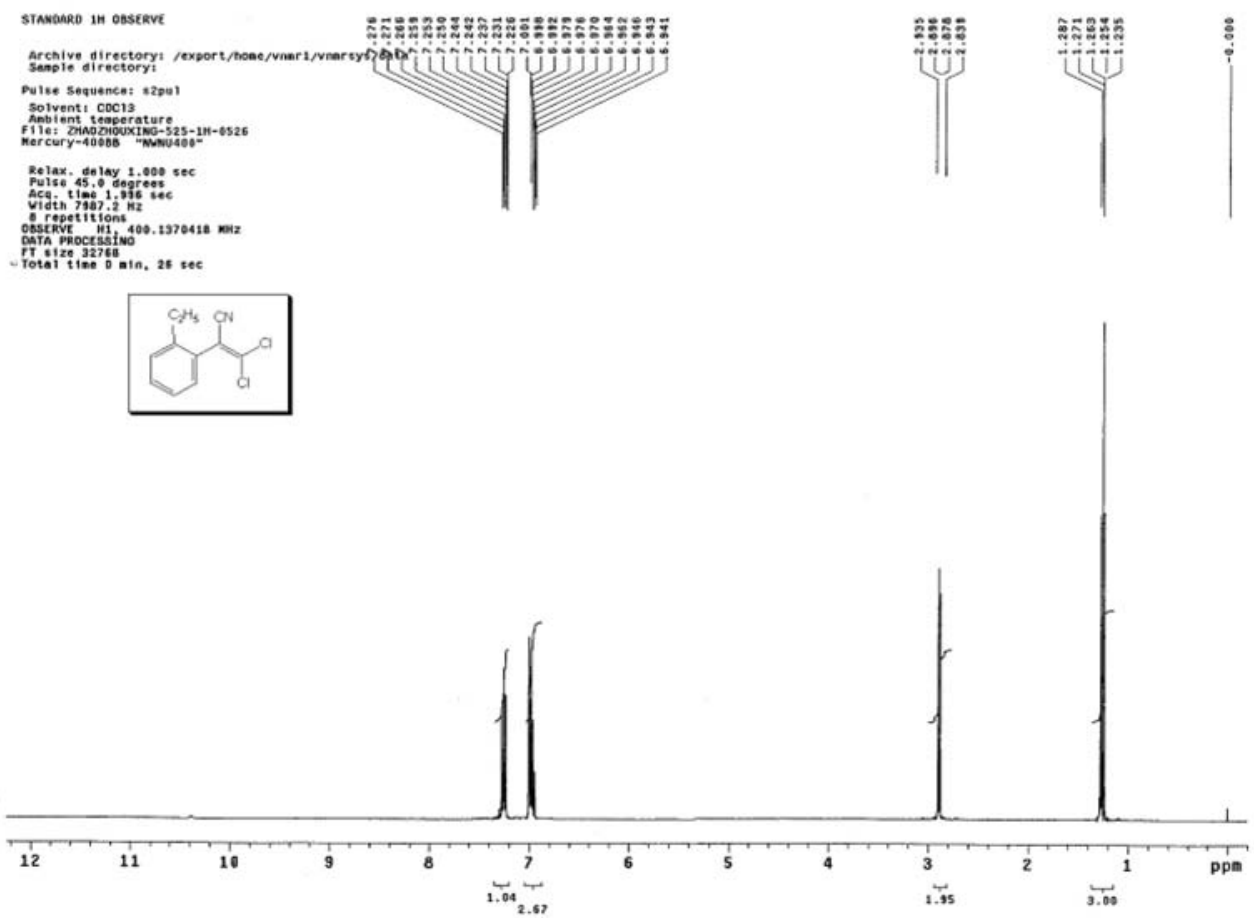

Figure S23. ${ }^{1} \mathrm{H}$ NMR spectrum of 2-(2-ethylphenyl)-3,3-dichloroacrylonitrile (1h). 


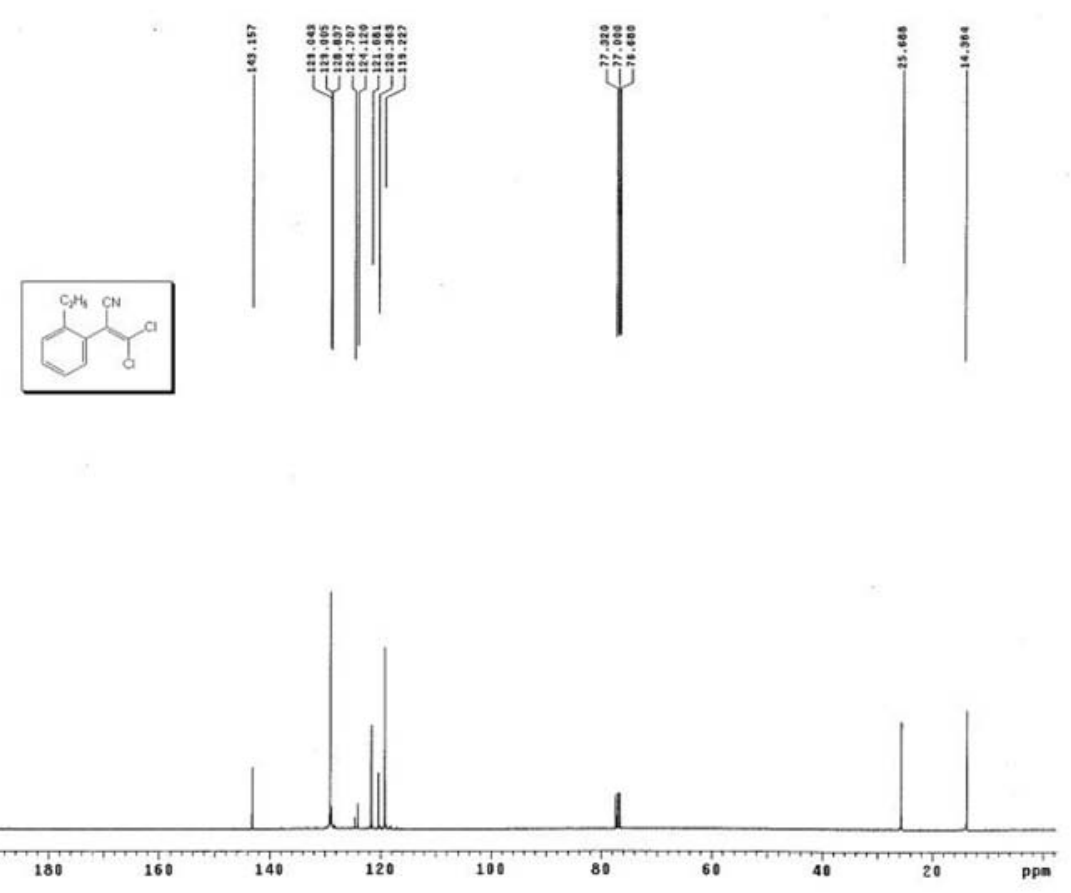

Figure S24. ${ }^{13} \mathrm{C}$ NMR spectrum of 2-(2-ethylphenyl)-3,3-dichloroacrylonitrile (1h).

\section{2-(3-Nitrophenyl)-3,3-dichloroacrylonitrile (1i)}

IR (KBr) $v_{\max } / \mathrm{cm}^{-1}: 3081,2924,2223,1614,1532,1348,1263,947 ;{ }^{1} \mathrm{H}$ NMR (CDCl $\left.3,400 \mathrm{MHz}\right): \delta 8.45$ (s, $\left.1 \mathrm{H}, \mathrm{Ar}-\mathrm{H}\right)$, 8.33 (d, 1H, J $8.0 \mathrm{~Hz}, \mathrm{Ar}-\mathrm{H}), 7.87$ (d, 1H, J $8.0 \mathrm{~Hz}, \mathrm{Ar}-\mathrm{H}), 7.70$ (t, 1H, J 8.0 Hz, Ar-H); ${ }^{13} \mathrm{C}$ NMR $\left(\mathrm{CDCl}_{3}, 100 \mathrm{MHz}\right): \delta$ $148.2,140.1,134.6,132.2,130.2,124.9,123.9,114.7,113.4$.

\section{Digilab Merlin}

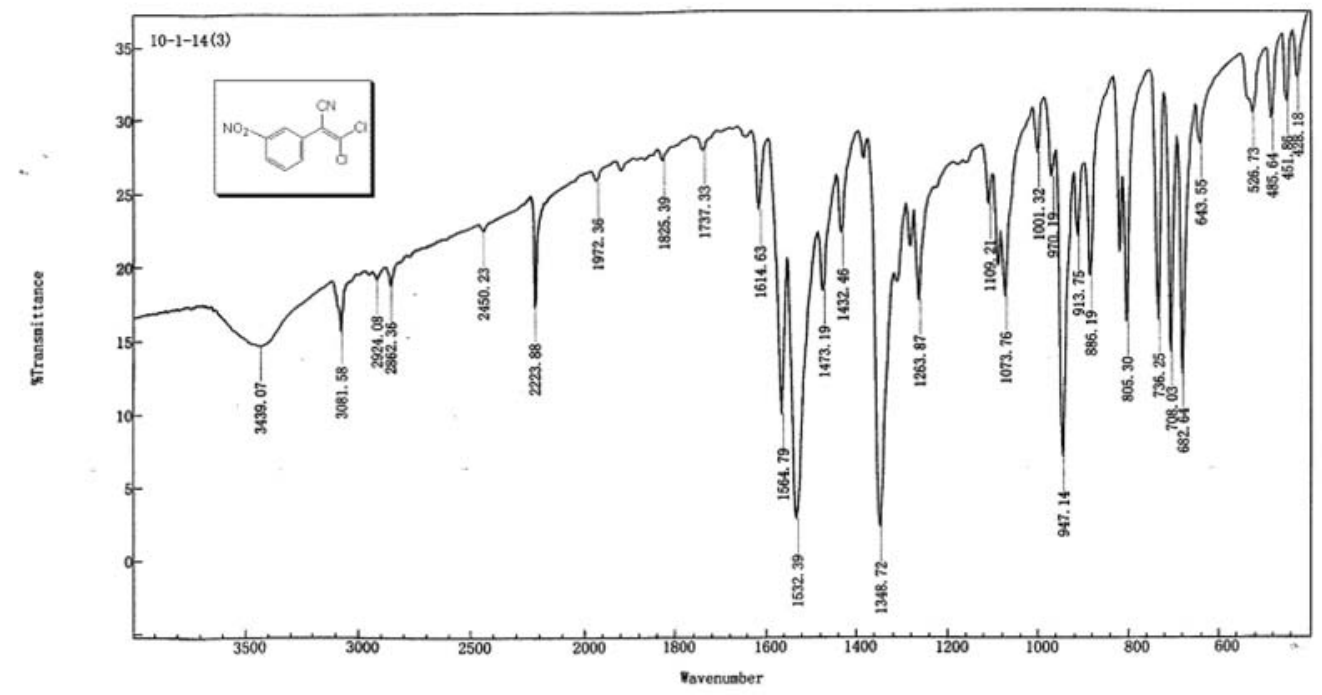

Figure S25. IR spectrum of 2-(3-nitrophenyl)-3,3-dichloroacrylonitrile (1i). 


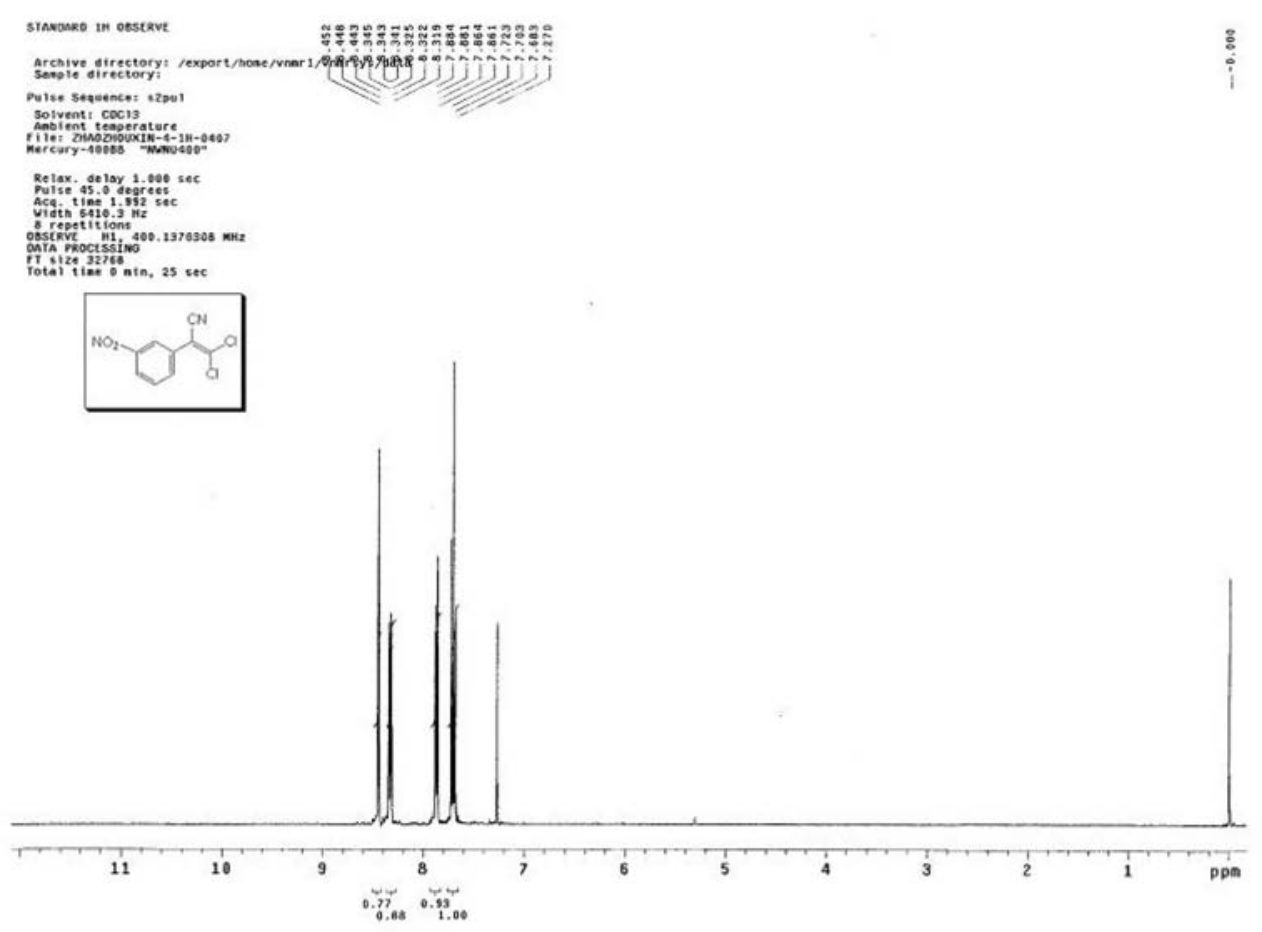

Figure S26. 'H NMR spectrum of 2-(3-nitrophenyl)-3,3-dichloroacrylonitrile (1i).
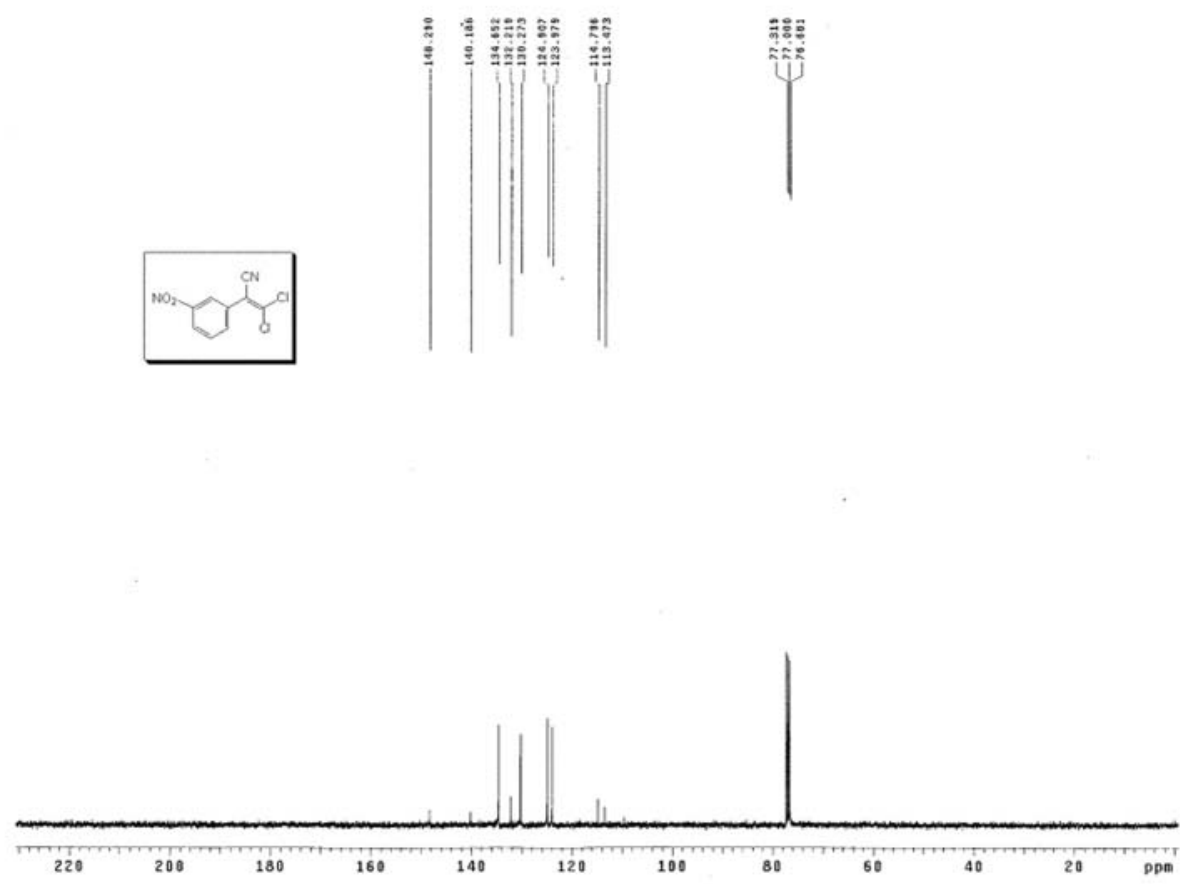

Figure S27. ${ }^{13} \mathrm{C}$ NMR spectrum of 2-(3-nitrophenyl)-3,3-dichloroacrylonitrile (1i). 


\section{2-(4-Nitrophenyl)-3,3-dichloroacrylonitrile ( $1 \mathbf{j})$}

IR (KBr) $v_{\max } / \mathrm{cm}^{-1}: 3049,2923,2235,1555,1481,1377,1226,1024,939,748 ;{ }^{1} \mathrm{H}$ NMR $\left(\mathrm{CDCl}_{3}, 400 \mathrm{MHz}\right): \delta 8.33$ (d, $J 9.2 \mathrm{~Hz}, 2 \mathrm{H}, \mathrm{Ar}-\mathrm{H}), 7.74$ (d, 2H, $J 9.2 \mathrm{~Hz}, \mathrm{Ar}-\mathrm{H}) ;{ }^{13} \mathrm{C} \mathrm{NMR}\left(\mathrm{CDCl}_{3}, 100 \mathrm{MHz}\right): \delta 148.3,140.2,136.7,130.0,124.1$, 114.7, 113.8 .

\section{Digilab Merlin}

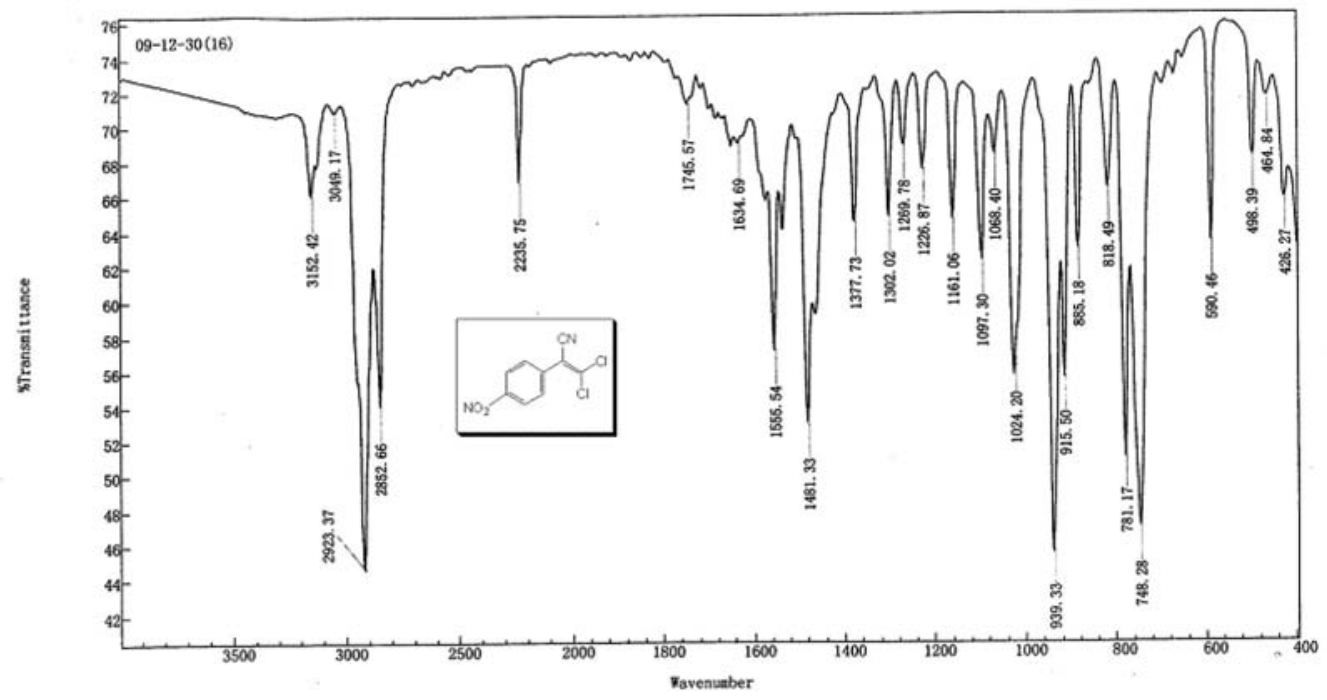

Figure S28. IR spectrum of 2-(4-nitrophenyl)-3,3-dichloroacrylonitrile (1j).

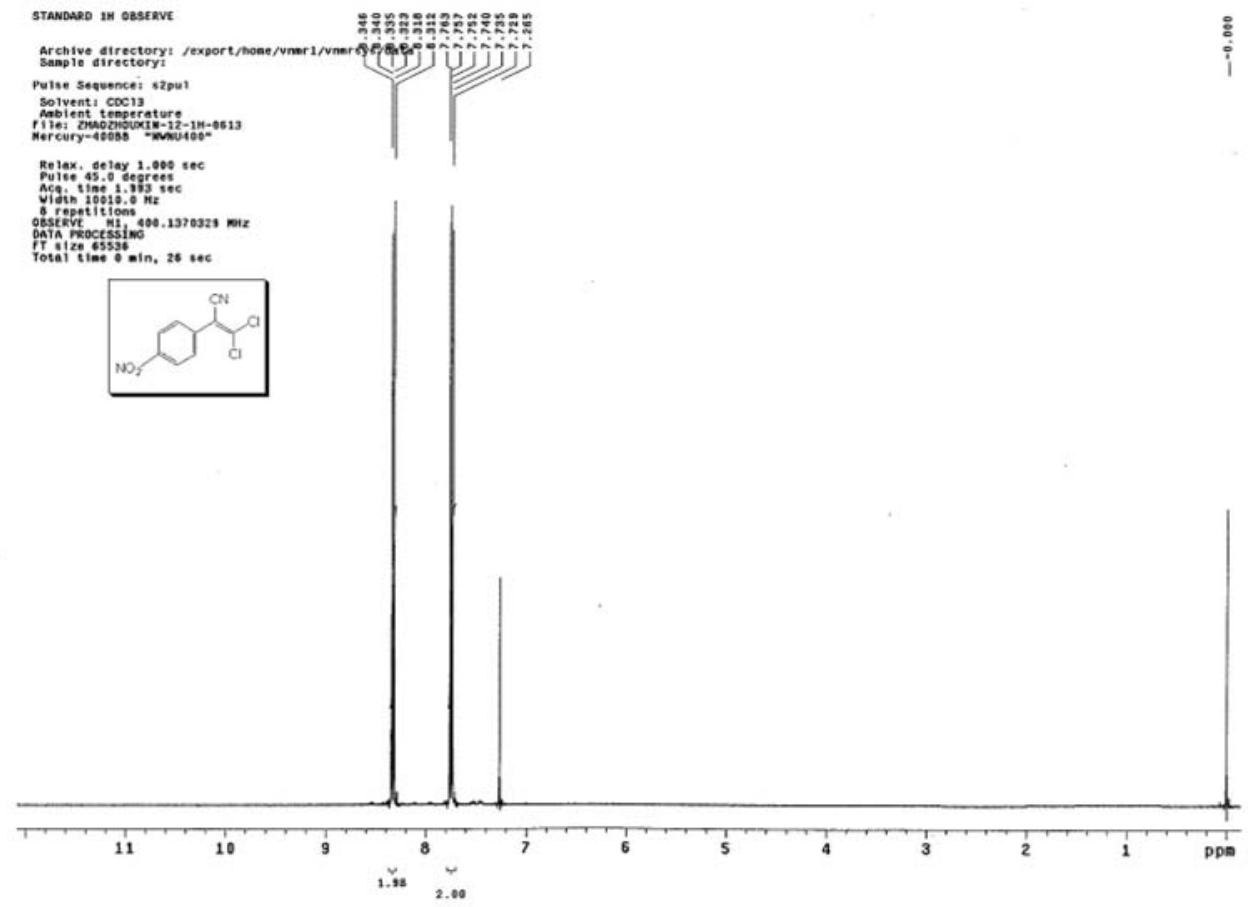

Figure S29. 'H NMR spectrum of 2-(4-nitrophenyl)-3,3-dichloroacrylonitrile (1j). 


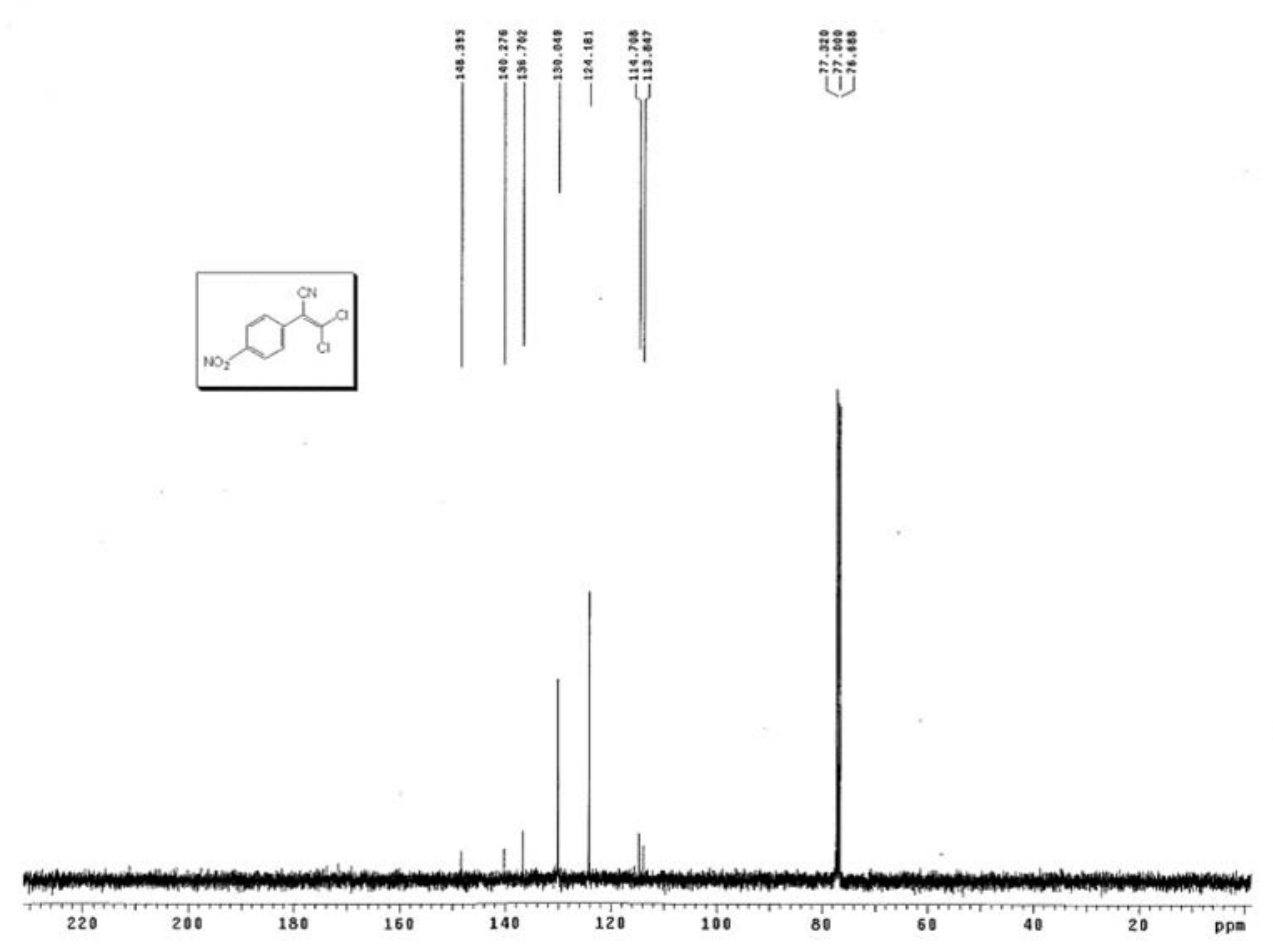

Figure S30. ${ }^{13} \mathrm{C}$ NMR spectrum of 2-(4-nitrophenyl)-3,3-dichloroacrylonitrile (1j).

\section{2-(4-Ethoxyphenyl)-3,3-dichloroacrylonitrile (1k)}

IR (KBr) $v_{\max } / \mathrm{cm}^{-1}: 3059,2988,2218,1606,1510,1265,924 ;{ }^{1} \mathrm{H}$ NMR $\left(\mathrm{CDCl}_{3}, 400 \mathrm{MHz}\right): \delta 7.46(\mathrm{~d}, 2 \mathrm{H}, J 8.8 \mathrm{~Hz}$, Ar-H), 6.93 (d, $2 \mathrm{H}, J 8.8 \mathrm{~Hz}, \mathrm{Ar}-\mathrm{H}), 4.06\left(\mathrm{q}, 2 \mathrm{H}, J 6.8 \mathrm{~Hz}, \mathrm{CH}_{2}\right), 1.43\left(\mathrm{t}, 3 \mathrm{H}, J 6.8 \mathrm{~Hz}, \mathrm{CH}_{3}\right) ;{ }^{13} \mathrm{C} \mathrm{NMR}\left(\mathrm{CDCl}_{3}, 100 \mathrm{MHz}\right)$ : $\delta 160.0,135.3,130.2,122.6,115.8,115.2,114.6,63.6,14.6$.

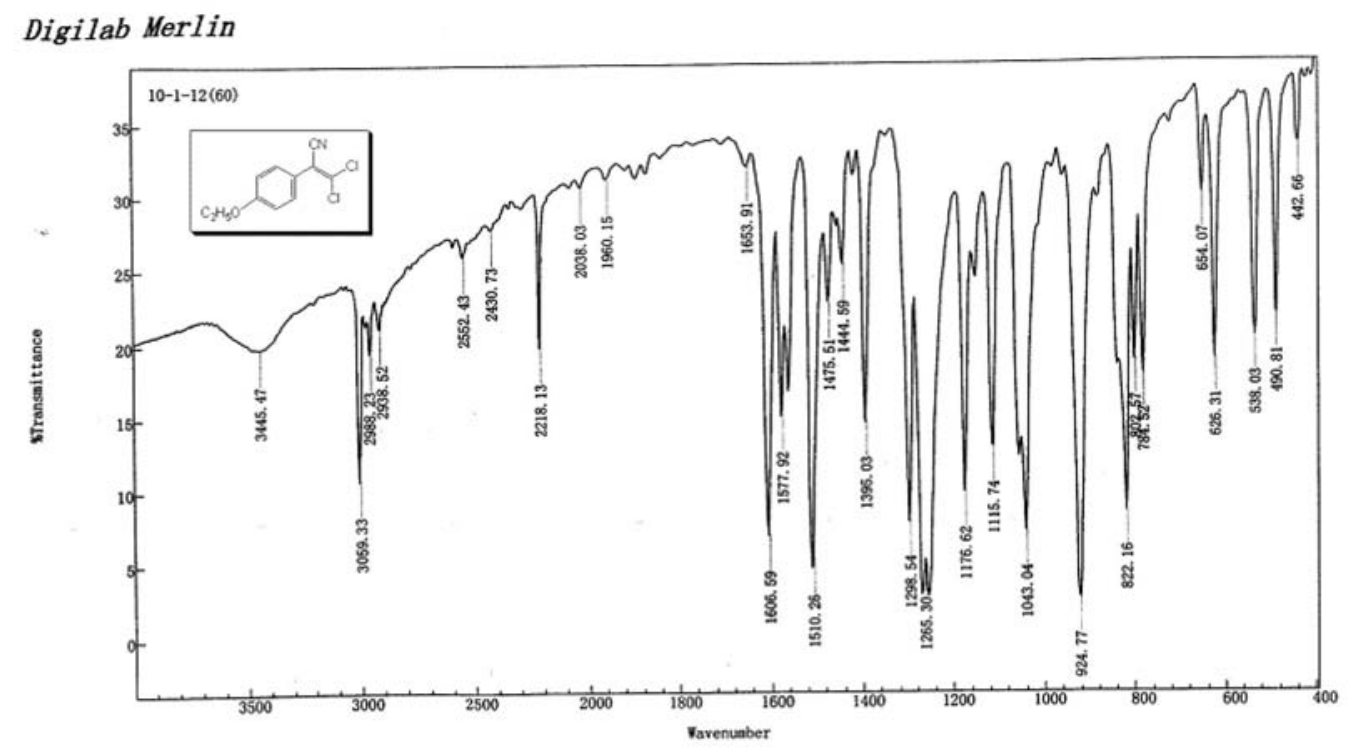

Figure S31. IR spectrum of 2-(4-ethoxyphenyl)-3,3-dichloroacrylonitrile (1k). 


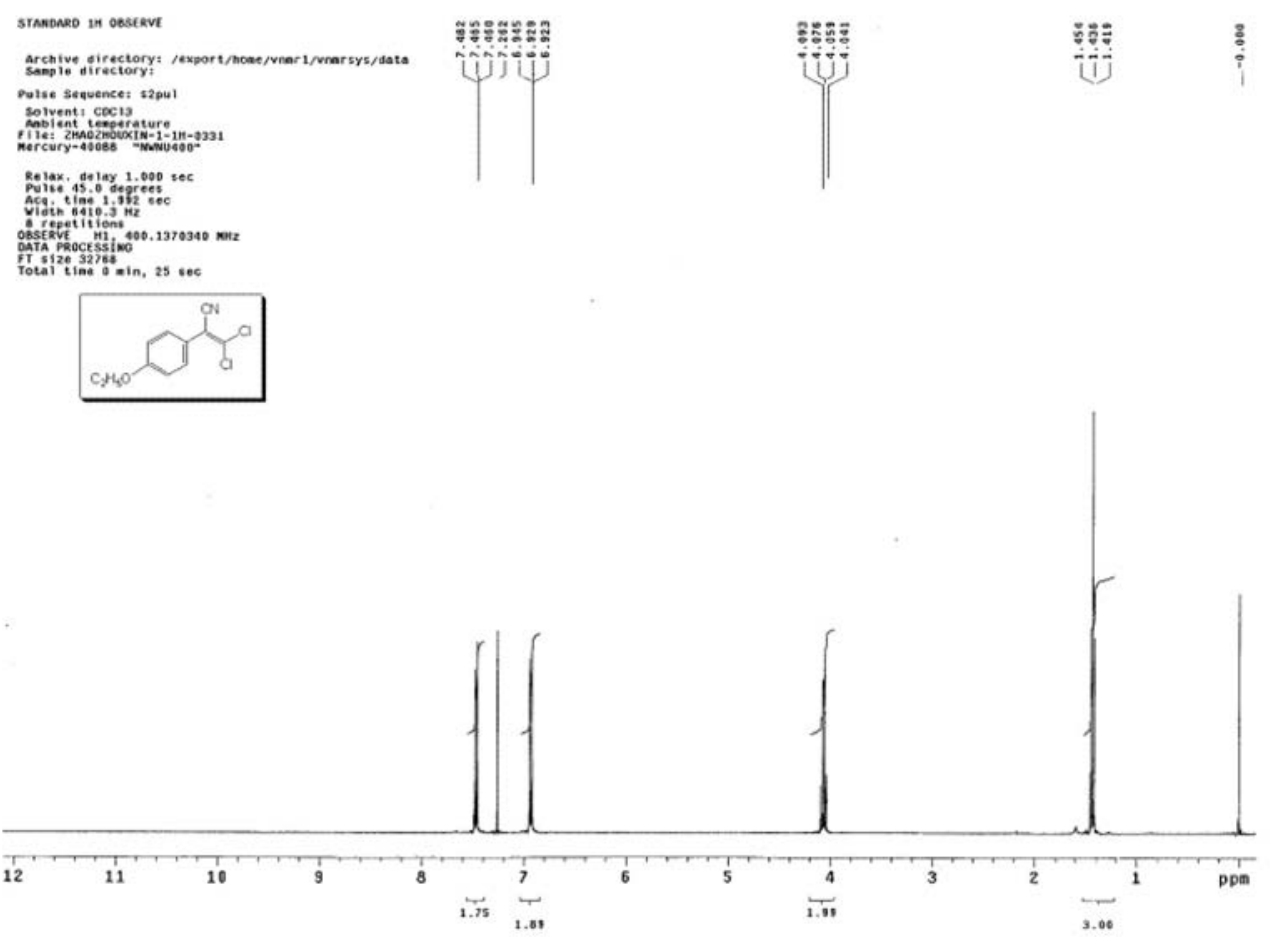

Figure S32. 'H NMR spectrum of 2-(4-ethoxyphenyl)-3,3-dichloroacrylonitrile (1k).
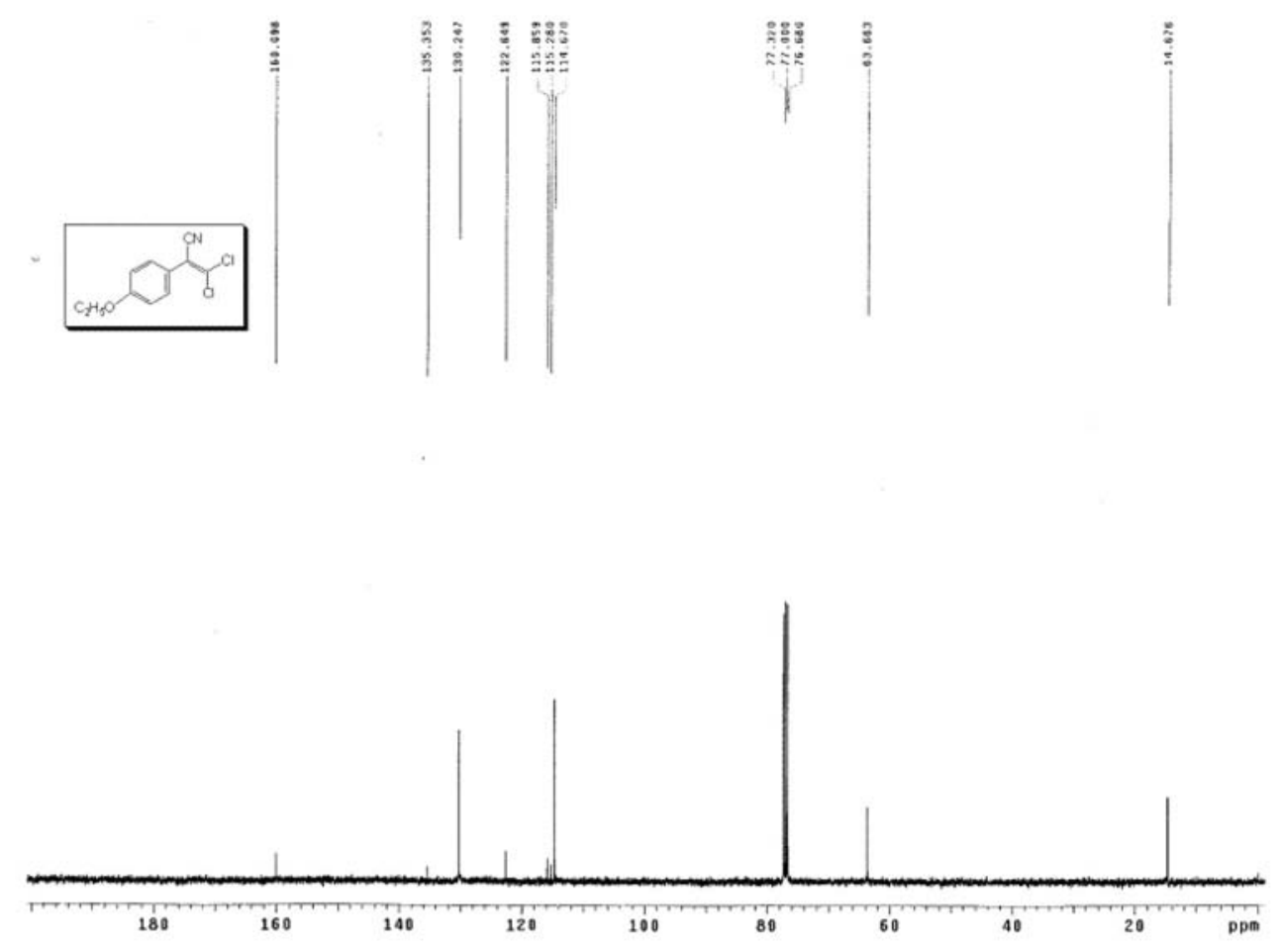

Figure S33. ${ }^{13} \mathrm{C}$ NMR spectrum of 2-(4-ethoxyphenyl)-3,3-dichloroacrylonitrile (1k). 
2-(4-Methoxyphenyl)-3,3-dichloroacrylonitrile (1l)

IR (KBr) $v_{\max } / \mathrm{cm}^{-1}: 3067,2988,2218,1589,1465,1287,854,765 ;{ }^{1} \mathrm{H}$ NMR $\left(\mathrm{CDCl}_{3}, 400 \mathrm{MHz}\right): \delta 7.48(\mathrm{~d}, 2 \mathrm{H}, J 9.2$ $\mathrm{Hz}, \mathrm{Ar}-\mathrm{H}), 6.95$ (d, $2 \mathrm{H}, J 9.2 \mathrm{~Hz}, \mathrm{Ar}-\mathrm{H}), 3.84\left(\mathrm{~s}, 3 \mathrm{H}, \mathrm{CH}_{3}\right) ;{ }^{13} \mathrm{C} \mathrm{NMR}\left(\mathrm{CDCl}_{3}, 100 \mathrm{MHz}\right): \delta 160.7,135.4,130.2,122.8$, $115.7,115.2,114.2,55.3$.

\section{Digilab Merlin}

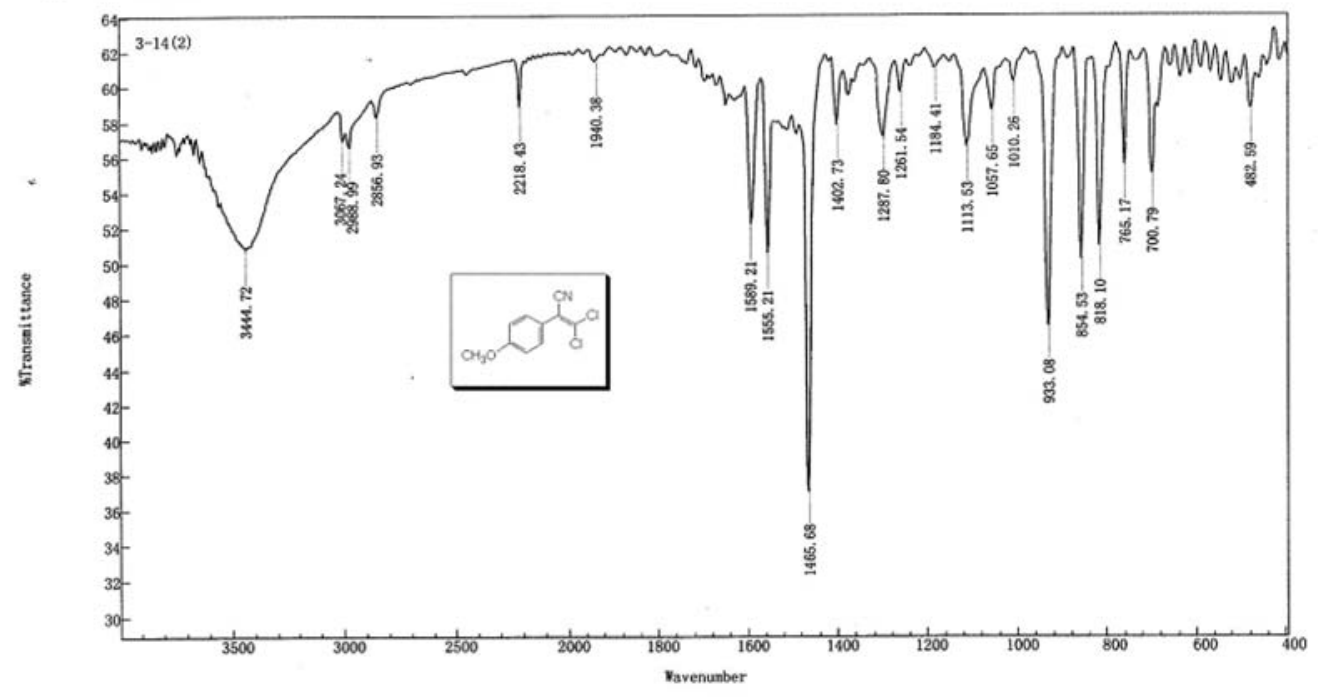

Figure S34.IR spectrum of 2-(4-methoxyphenyl)-3,3-dichloroacrylonitrile (11).

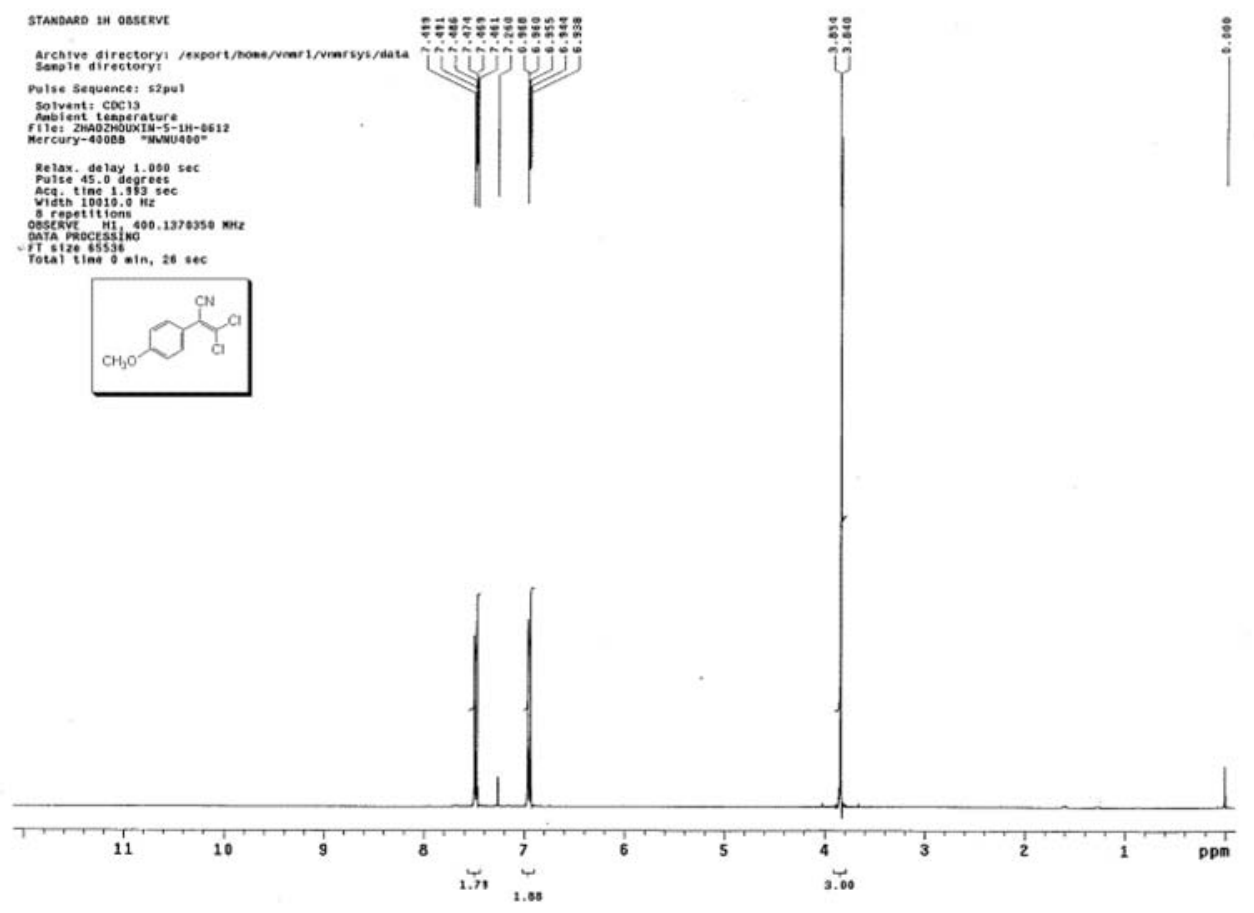

Figure S35. ${ }^{1} \mathrm{H}$ NMR spectrum of 2-(4-methoxyphenyl)-3,3-dichloroacrylonitrile (11). 


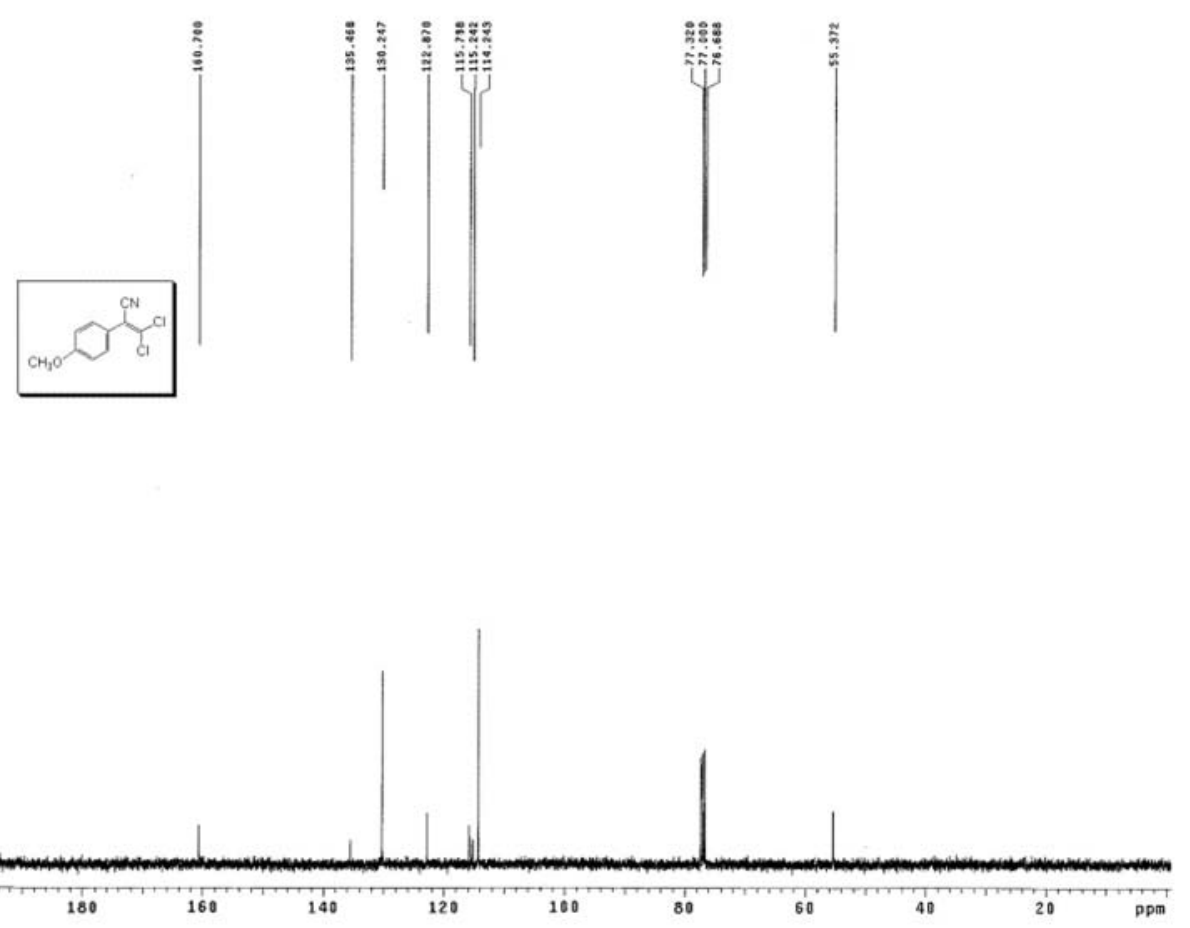

Figure S36. ${ }^{13} \mathrm{C}$ NMR spectrum of 2-(4-methoxyphenyl)-3,3-dichloroacrylonitrile (11).

\section{2-(4-Iodophenyl)-3,3-dichloroacrylonitrile (1m)}

IR (KBr) $v_{\max } / \mathrm{cm}^{-1}: 3065,2220,1572,1481,1288,1276,933,834 ;{ }^{1} \mathrm{H}$ NMR $\left(\mathrm{CDCl}_{3}, 400 \mathrm{MHz}\right): \delta 7.79(\mathrm{~d}, 2 \mathrm{H}, J 8.8$ $\mathrm{Hz}, \mathrm{Ar}-\mathrm{H}), 7.26$ (d, 2H, J 8.8 Hz, Ar-H); ${ }^{13} \mathrm{C} \mathrm{NMR}\left(\mathrm{CDCl}_{3}, 100 \mathrm{MHz}\right): \delta$ 138.1, 137.8, 130.3, 130.1, 115.2, 114.7, 96.6.

\section{Digilab Merlin}

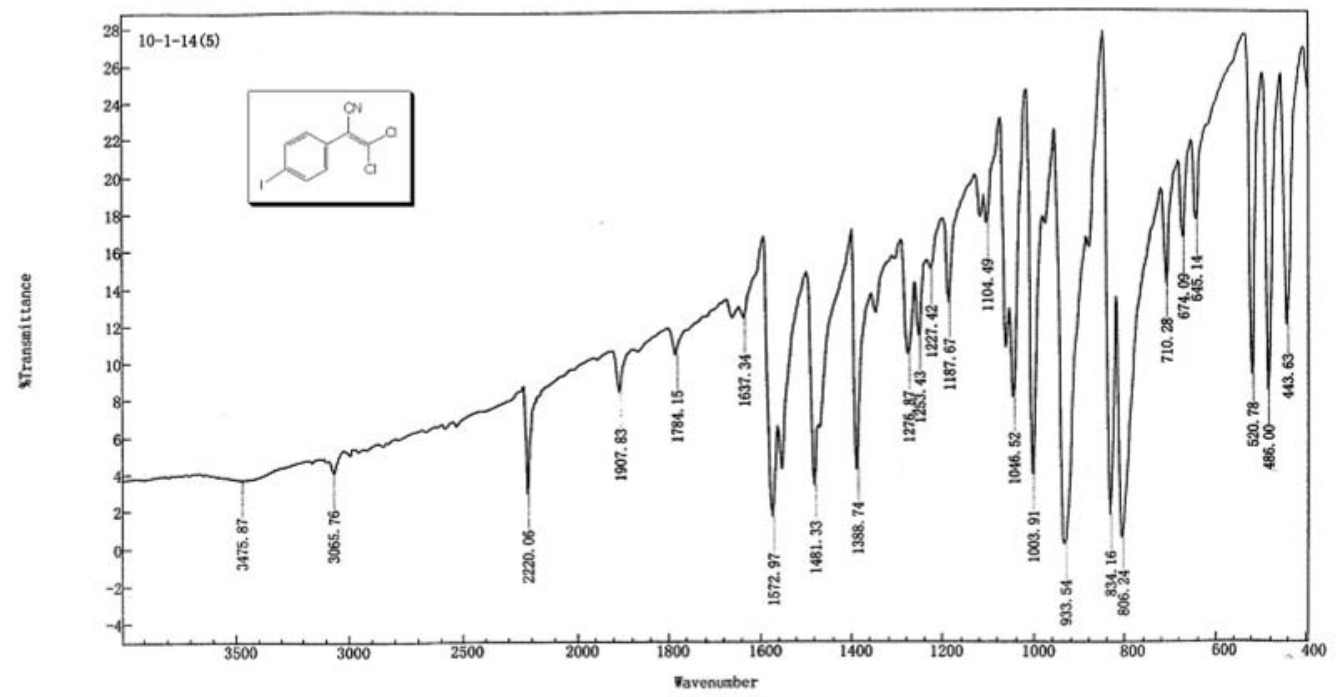

Figure S37. IR spectrum of 2-(4-iodophenyl)-3,3-dichloroacrylonitrile (1m). 


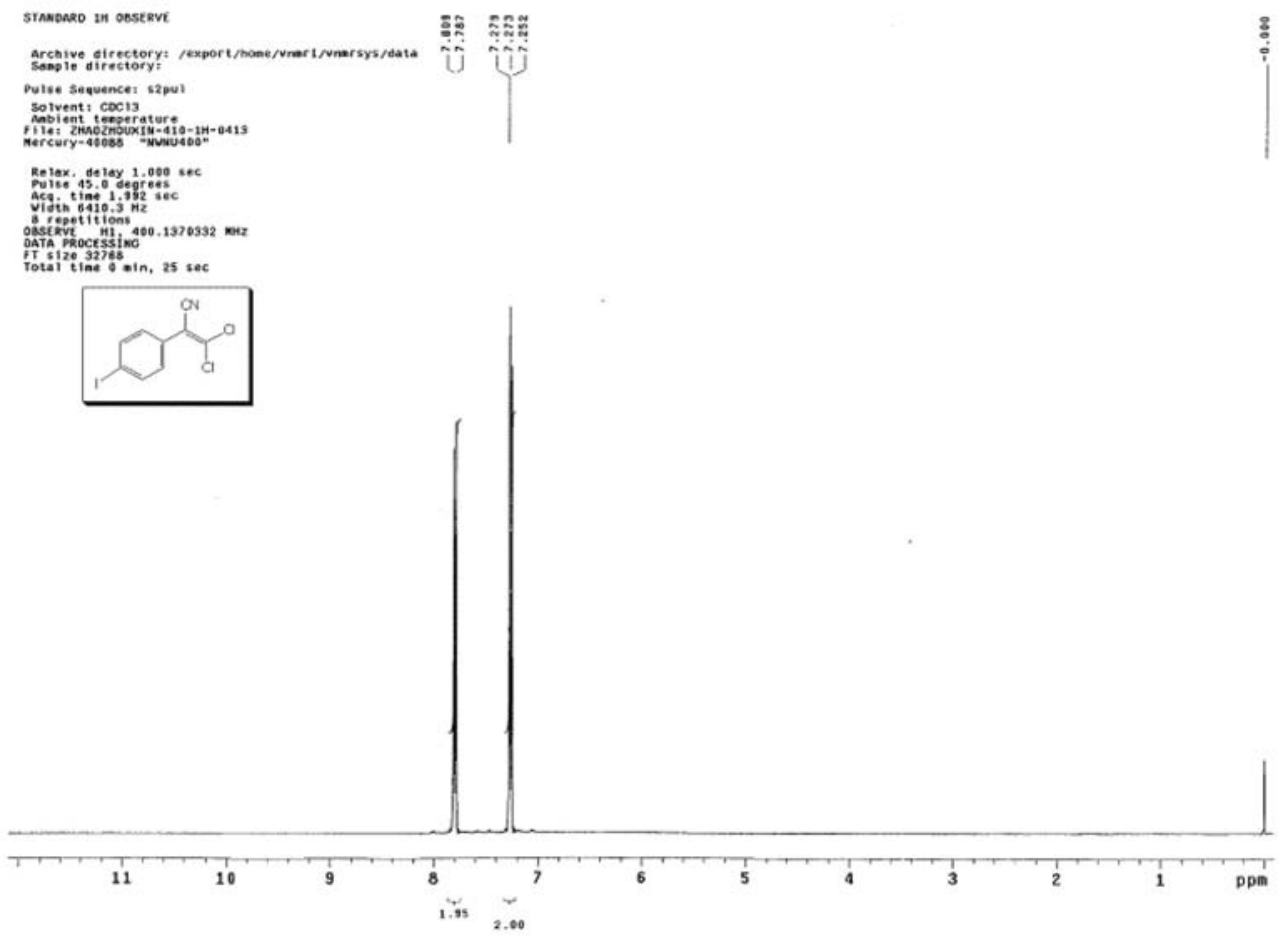

Figure S38. ${ }^{1} \mathrm{H}$ NMR spectrum of 2-(4-iodophenyl)-3,3-dichloroacrylonitrile (1m).
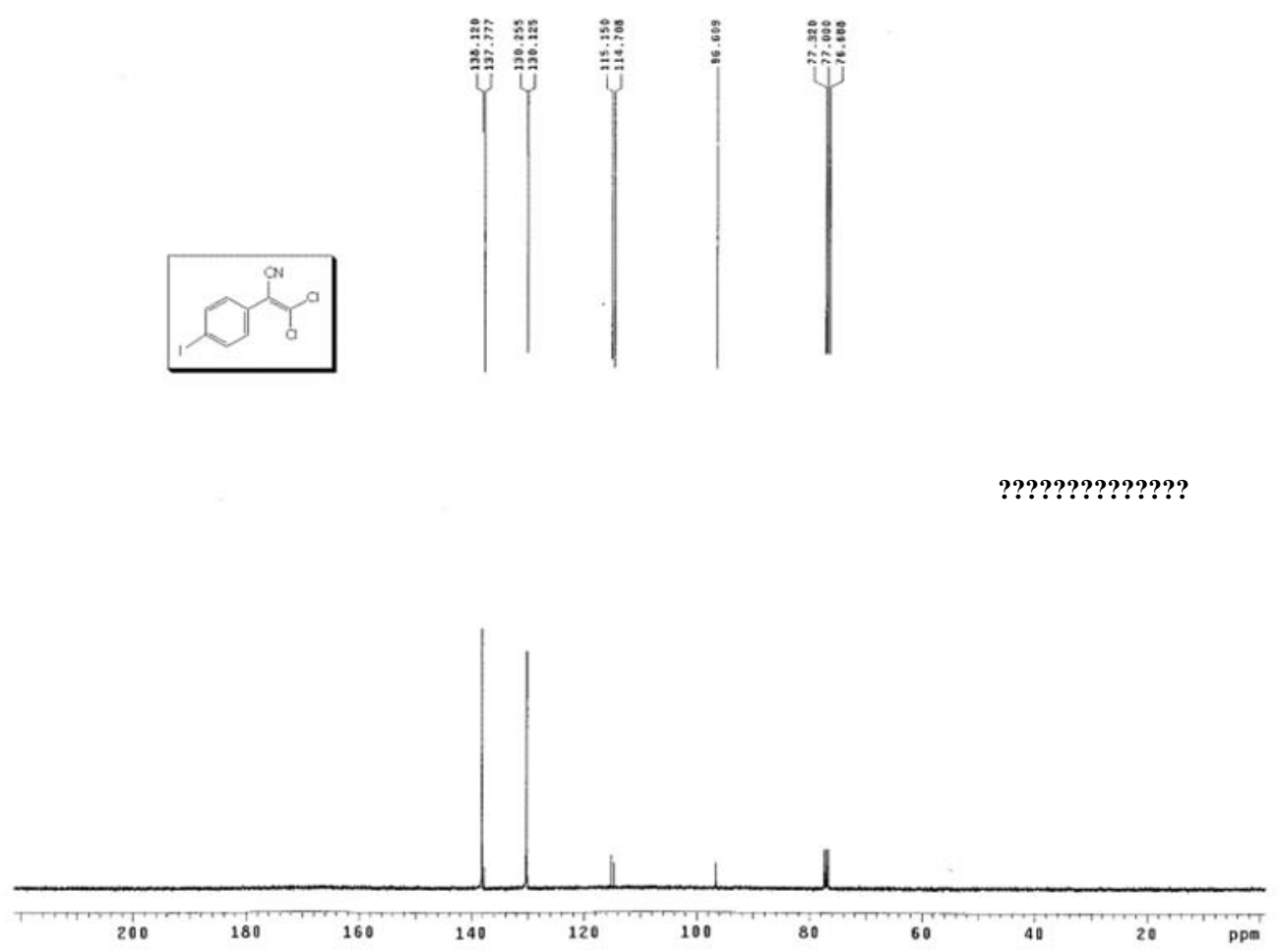

Figure S39. ${ }^{13} \mathrm{C}$ NMR spectrum of 2-(4-iodophenyl)-3,3-dichloroacrylonitrile (1m). 
2-(Furan-2-yl)-3,3-dichloroacrylonitrile (In)

IR (KBr) $v_{\max } / \mathrm{cm}^{-1}: 3109,2923,2220,1520,1350,931,858,817,698 ;{ }^{1} \mathrm{H} \mathrm{NMR}\left(\mathrm{CDCl}_{3}, 400 \mathrm{MHz}\right): \delta 7.76(\mathrm{~d}, 1 \mathrm{H}, J 1.6$ $\mathrm{Hz}, \mathrm{Fu}-\mathrm{H}), 7.04$ (d, 1H, J 4.4 Hz, Fu-H), 6.41 (dd, 1H, J $3.6 \mathrm{~Hz}, J 1.6 \mathrm{~Hz}, \mathrm{Fu}-\mathrm{H}),{ }^{13} \mathrm{C}$ NMR $\left(\mathrm{CDCl}_{3}, 100 \mathrm{MHz}\right): \delta 148.3$, 140.2, 136.6, 130.0, 124.1, 114.7, 113.8.

\section{Digilab Merlin}

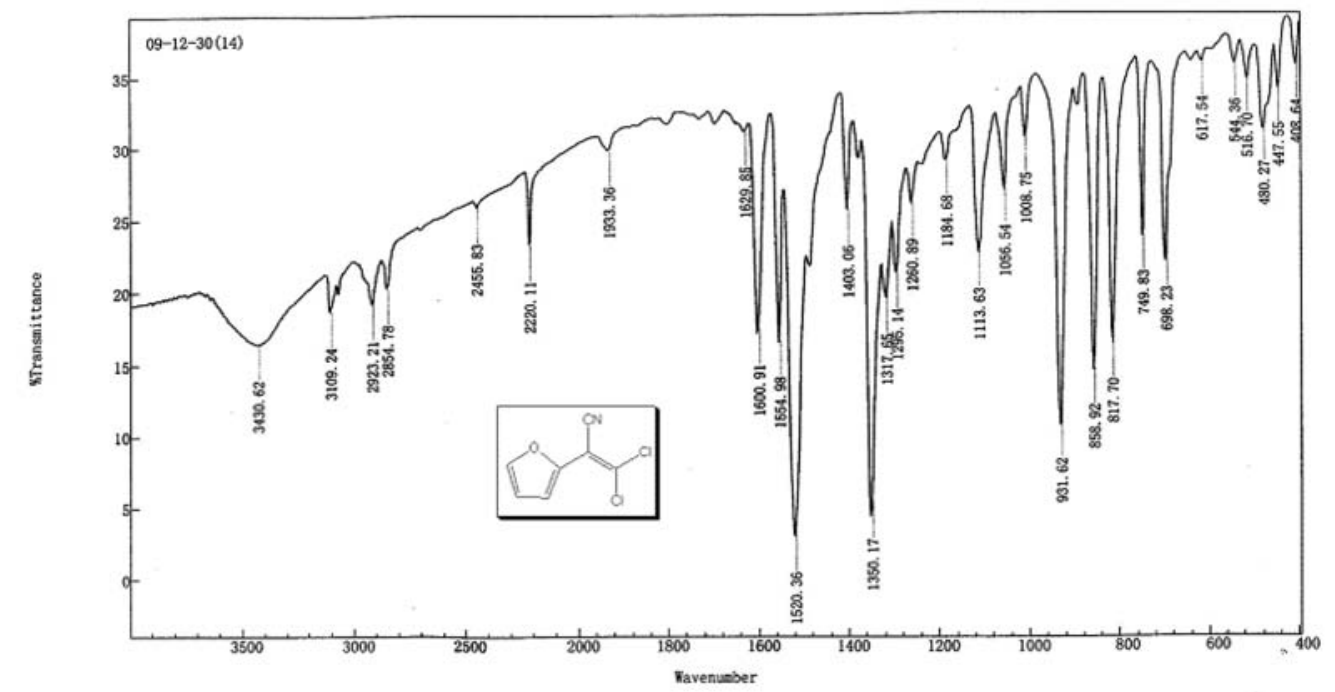

Figure S40. IR spectrum of 2-(furan-2-yl)-3,3-dichloroacrylonitrile (1n).

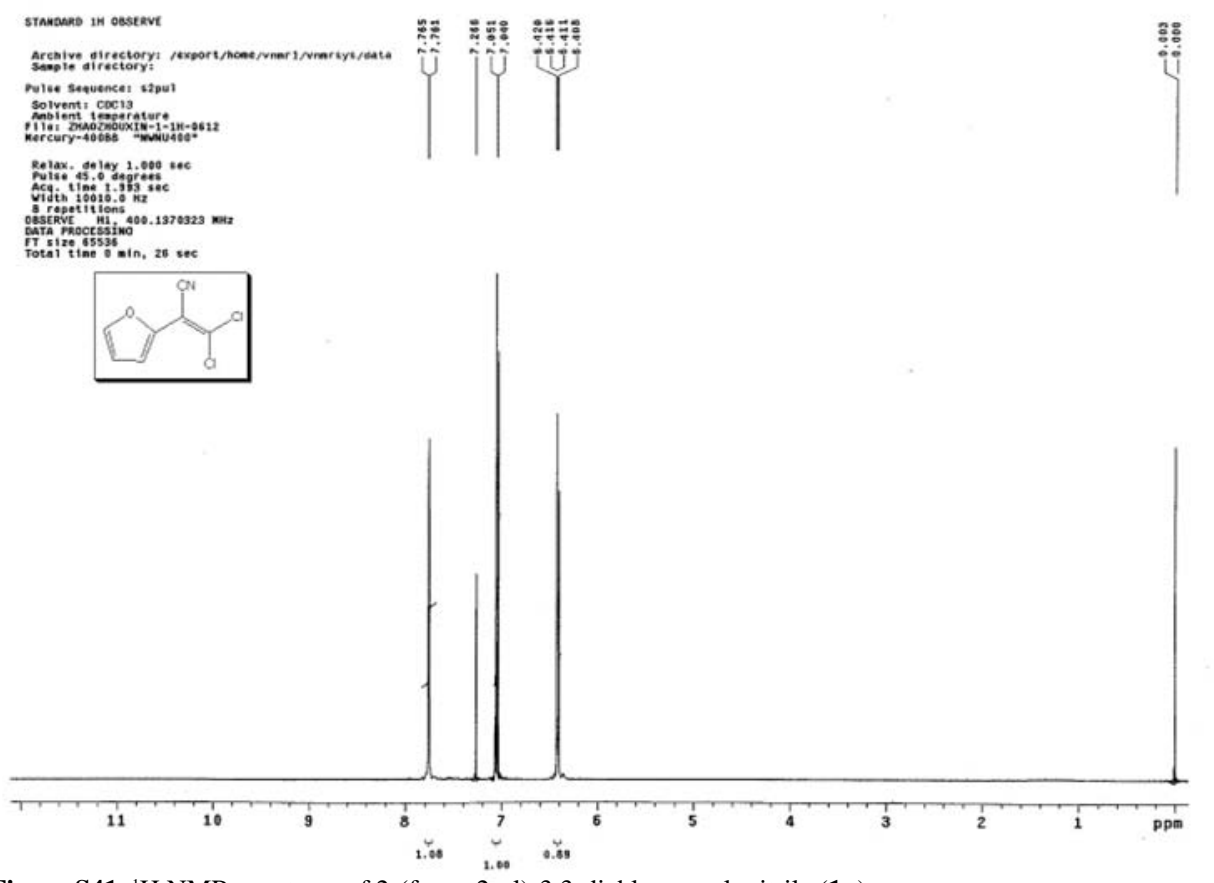

Figure S41. ${ }^{1} \mathrm{H}$ NMR spectrum of 2-(furan-2-yl)-3,3-dichloroacrylonitrile (1n). 


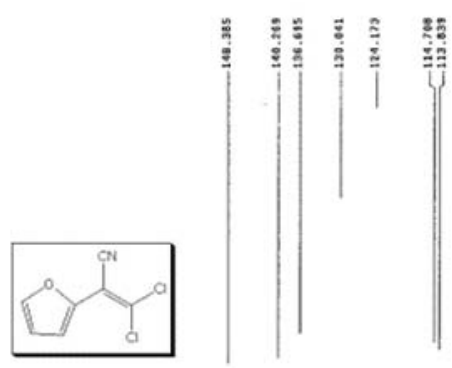

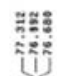

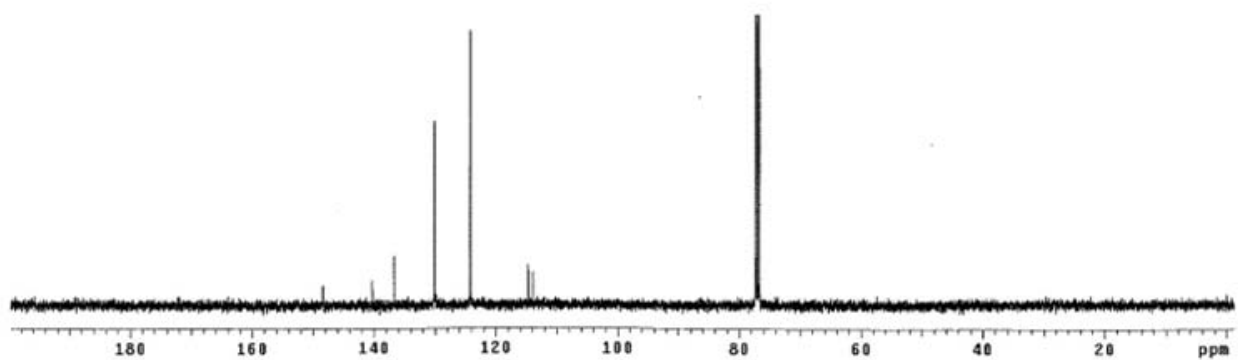

Figure S42. ${ }^{13} \mathrm{C}$ NMR spectrum of 2-(furan-2-yl)-3,3-dichloroacrylonitrile (1n).

\section{2-Phenyl-3,3-dibromoacrylonitrile (2a)}

IR (KBr) $v_{\max } / \mathrm{cm}^{-1}: 3062,2923,2216,1579,1487,1247,842,752,692 ;{ }^{1} \mathrm{H}$ NMR $\left(400 \mathrm{MHz}, \mathrm{CDCl}_{3}\right): \delta 7.45-7.48(\mathrm{~m}$, $5 \mathrm{H}, \mathrm{Ph}-\mathrm{H}) ;{ }^{13} \mathrm{C}$ NMR $\left(\mathrm{CDCl}_{3}, 100 \mathrm{MHz}\right): \delta 133.3,130.0,128.9,128.5,122.6,117.0,109.7$.

\section{Digilab Merlin}

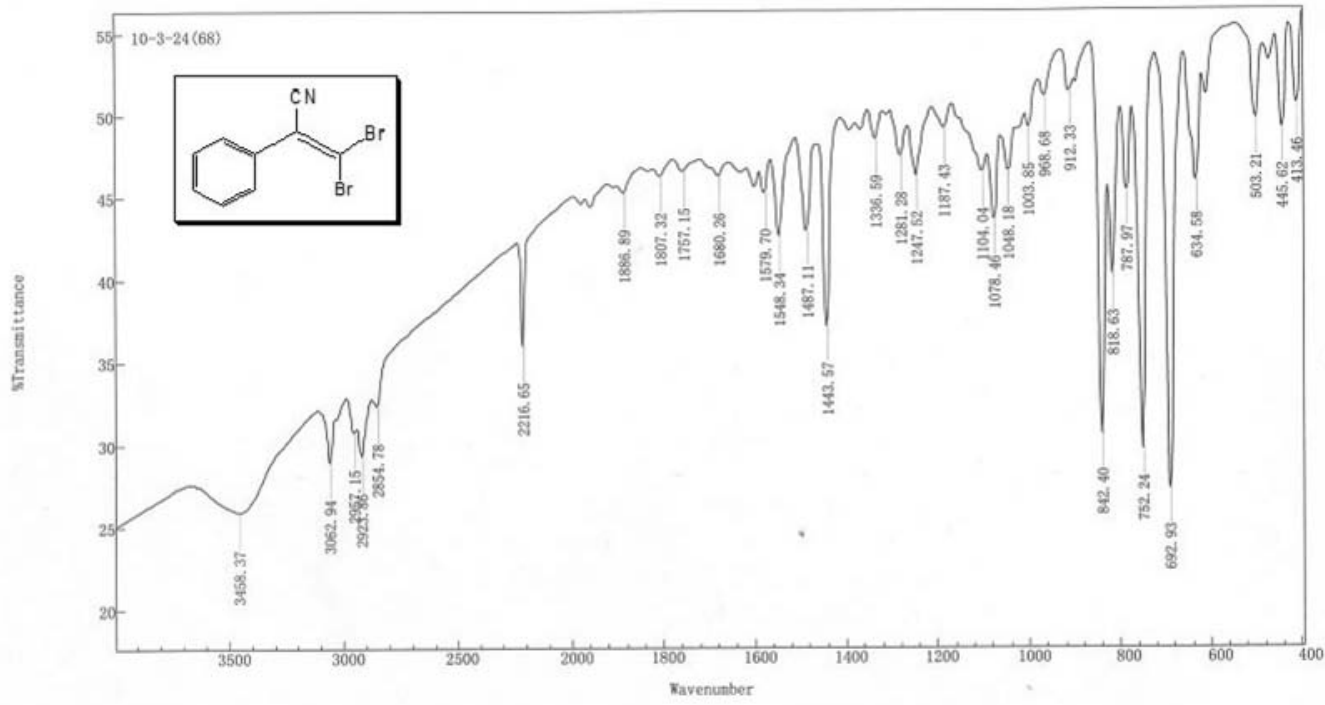

Figure S43. IR spectrum of 2-phenyl-3,3-dibromoacrylonitrile (2a). 


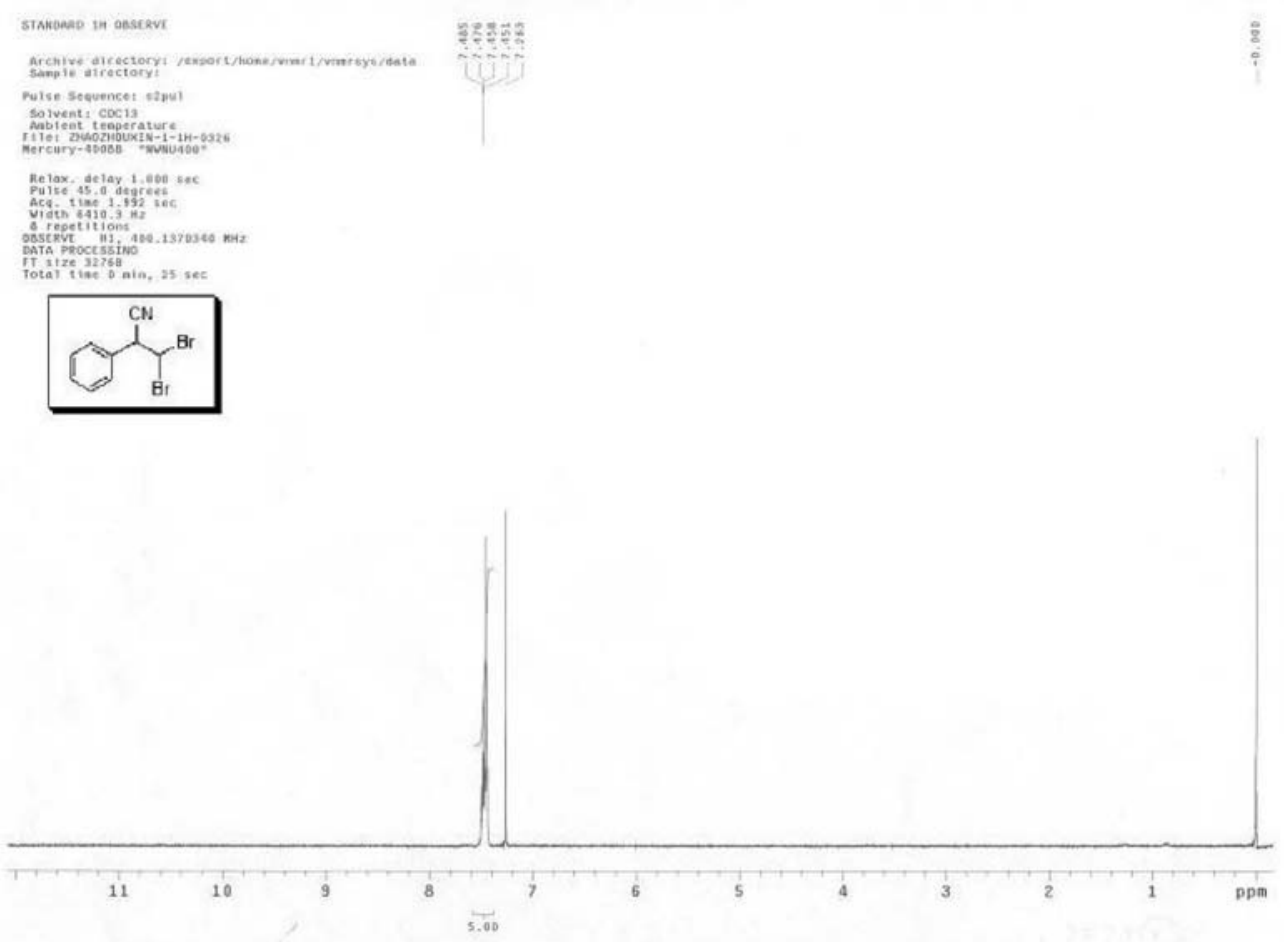

Figure S44. ${ }^{1} \mathrm{H}$ NMR spectrum of 2-phenyl-3,3-dibromoacrylonitrile (2a).

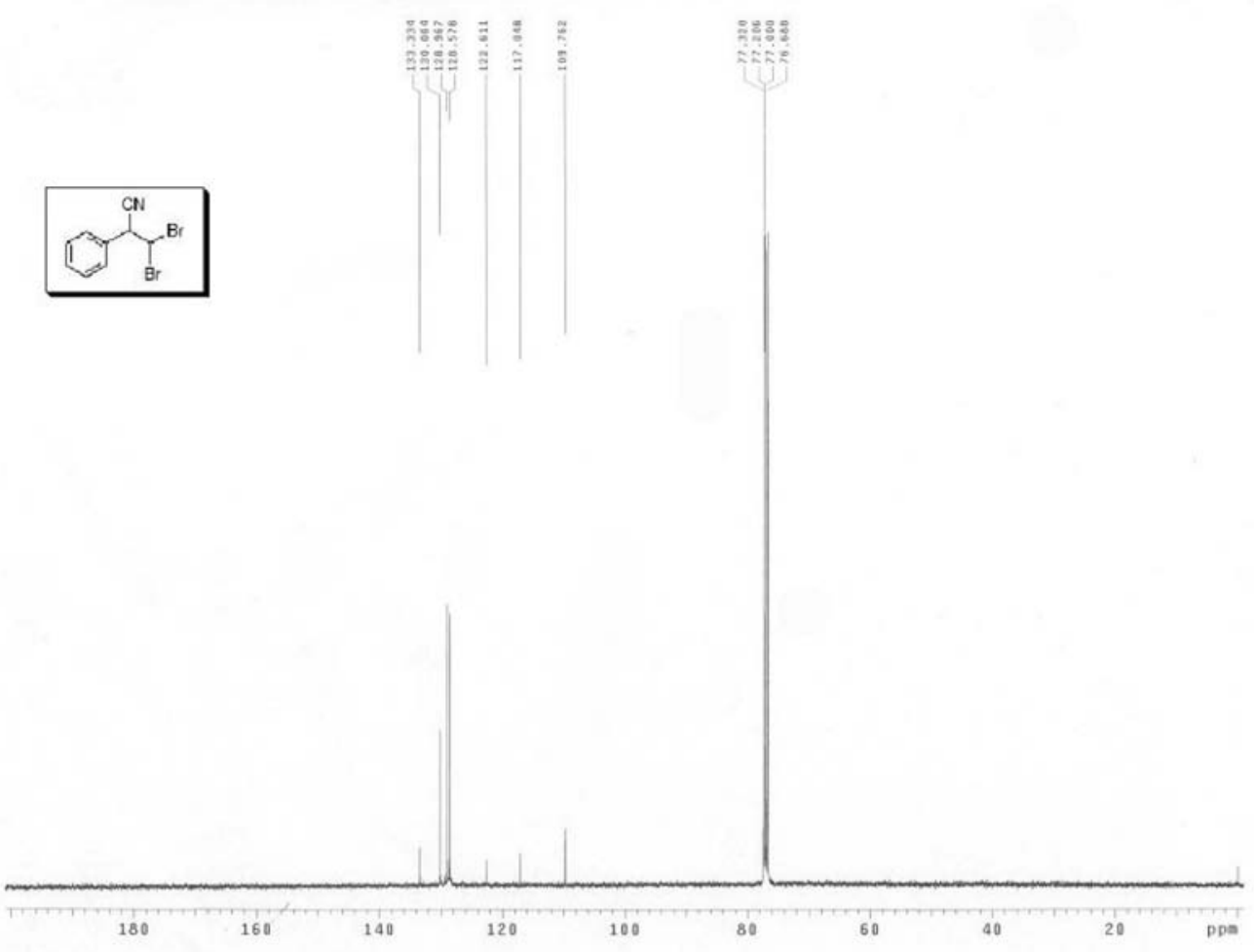

Figure S45. ${ }^{13} \mathrm{C}$ NMR spectrum of 2-phenyl-3,3-dibromoacrylonitrile (2a). 


\section{2-(2-Chlorophenyl)-3,3-dibromoacrylonitrile (2b)}

IR (KBr) $v_{\text {max }} / \mathrm{cm}^{-1}: 3069,2924,2218,1554,1467,1435,1287.1038,853,750 ;{ }^{1} \mathrm{H}$ NMR $\left(400 \mathrm{MHz}, \mathrm{CDCl}_{3}\right): \delta 7.50-7.25$ (m, 4H, Ph-H); $\left.{ }^{13} \mathrm{C} \mathrm{NMR} \mathrm{(CDCl} 3,100 \mathrm{MHz}\right): \delta 132.8,132.4,131.5,130.4,130.2,127.4,120.3,115.5,113.9$.

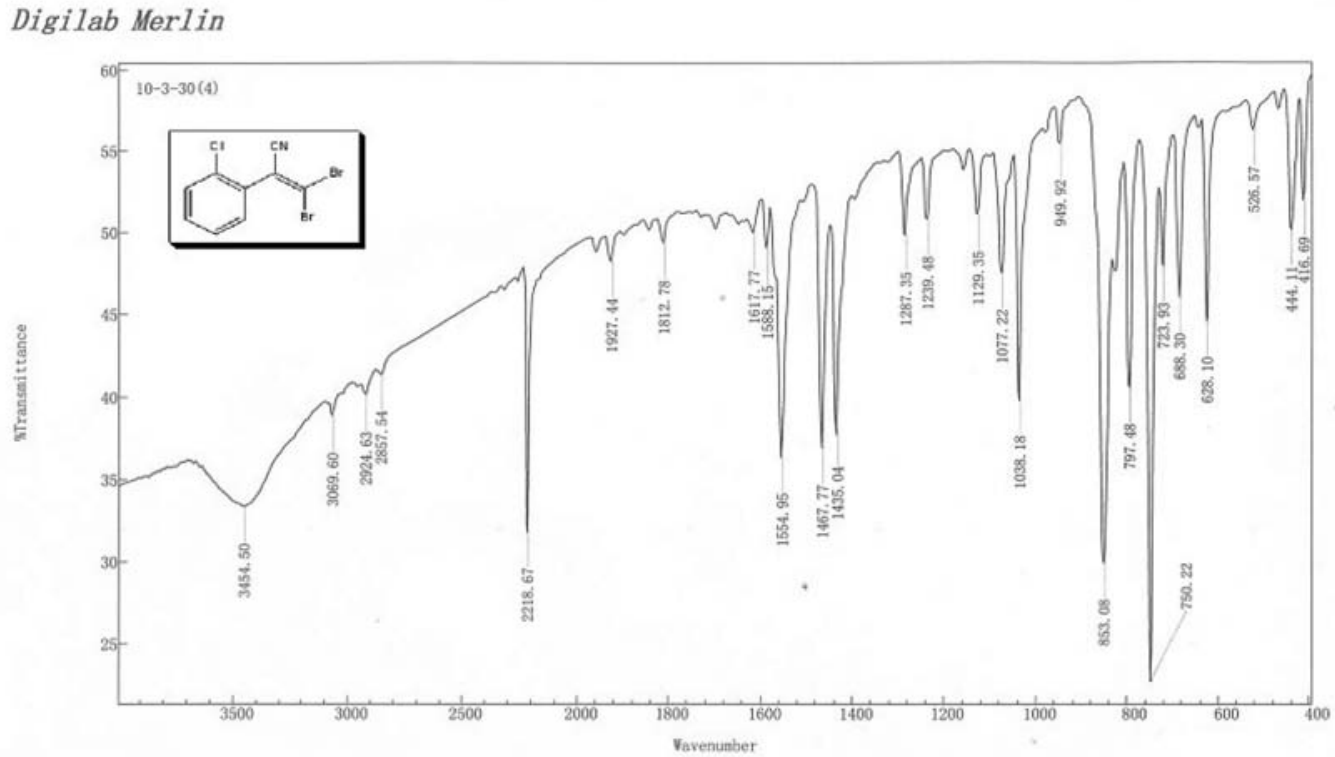

Figure S46. IR spectrum of 2-(2-chlorophenyl)-3,3-dibromoacrylonitrile (2b).

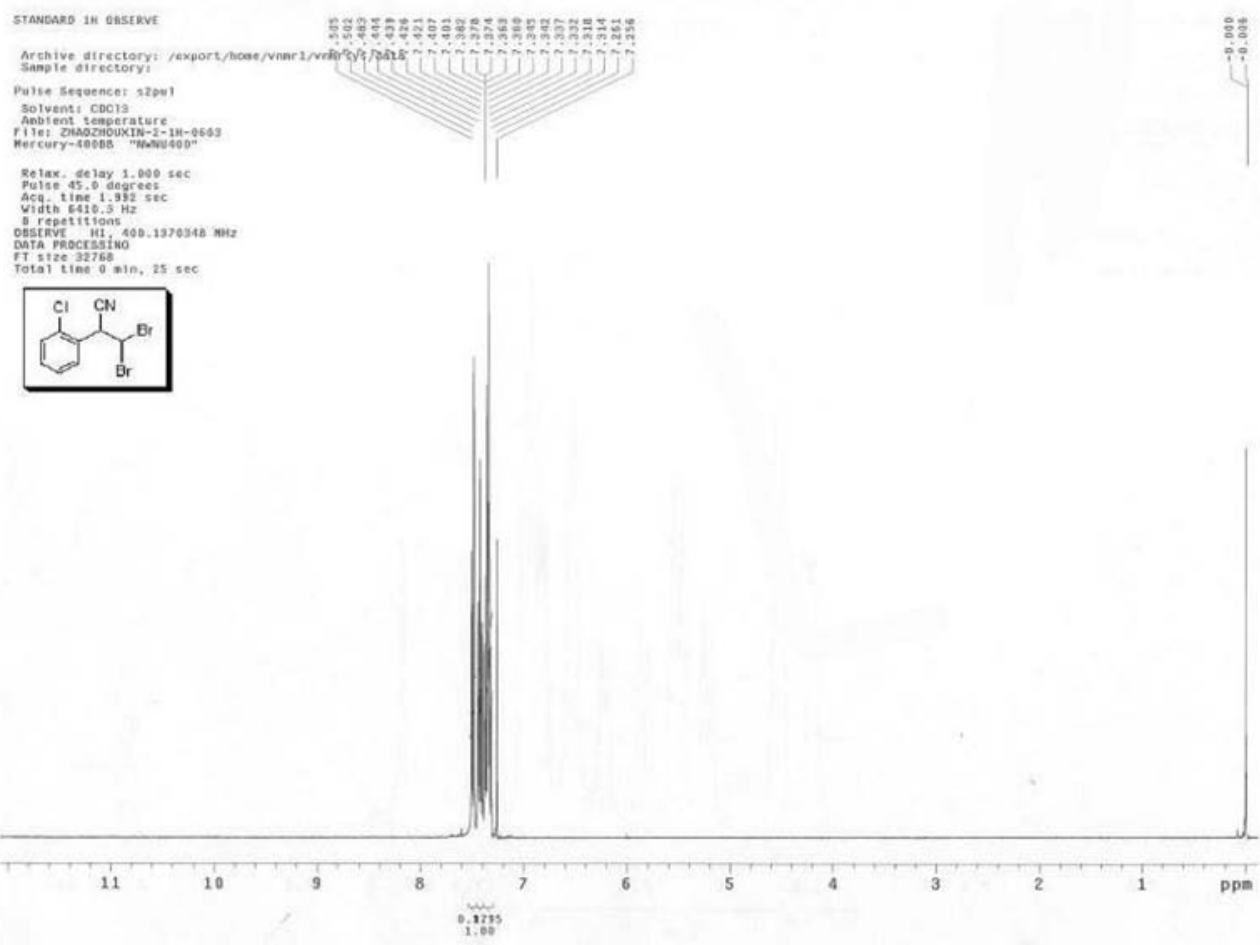

Figure S47. ${ }^{1} \mathrm{H}$ NMR spectrum of 2-(2-chlorophenyl)-3,3-dibromoacrylonitrile (2b). 

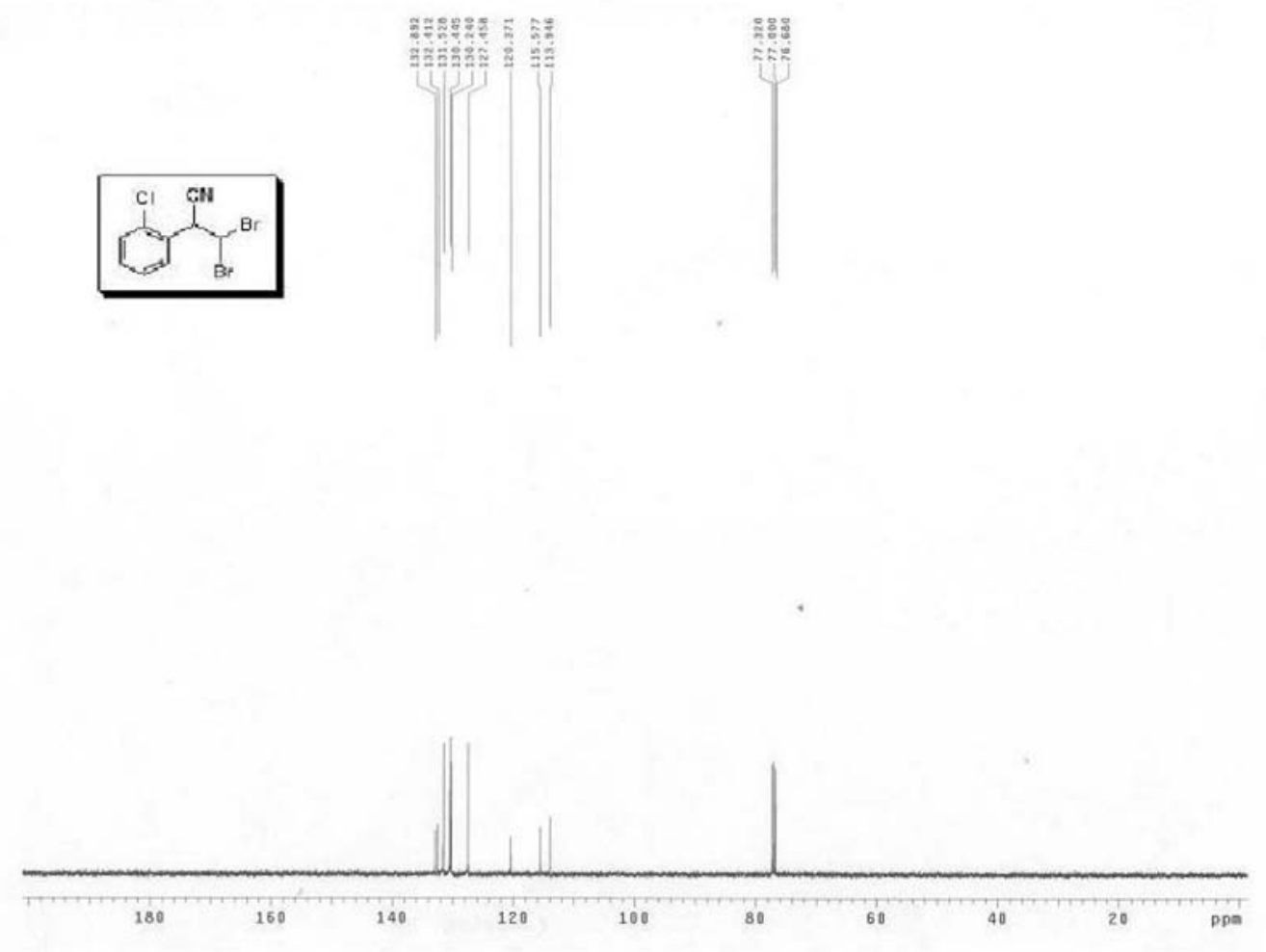

Figure S48. ${ }^{13} \mathrm{C}$ NMR spectrum of 2-(2-chlorophenyl)-3,3-dibromoacrylonitrile (2b).

\section{2-(3-Chlorophenyl)-3,3-dibromoacrylonitrile (2c)}

$\mathrm{IR}(\mathrm{KBr}) \mathrm{v}_{\max } / \mathrm{cm}^{-1}: 3061,2924,2218,1570,1469,1254.852,785 ;{ }^{1} \mathrm{H} \mathrm{NMR}\left(400 \mathrm{MHz}, \mathrm{CDCl}_{3}\right): \delta 7.48-7.35(\mathrm{~m}, 4 \mathrm{H}$, $\mathrm{Ph}-\mathrm{H}) ;{ }^{13} \mathrm{C}$ NMR $\left(\mathrm{CDCl}_{3}, 100 \mathrm{MHz}\right): \delta 134.9,134.7,130.2,130.2,128.6,126.7,121.2,116.6,111.1$.

\section{Digilab Merlin}

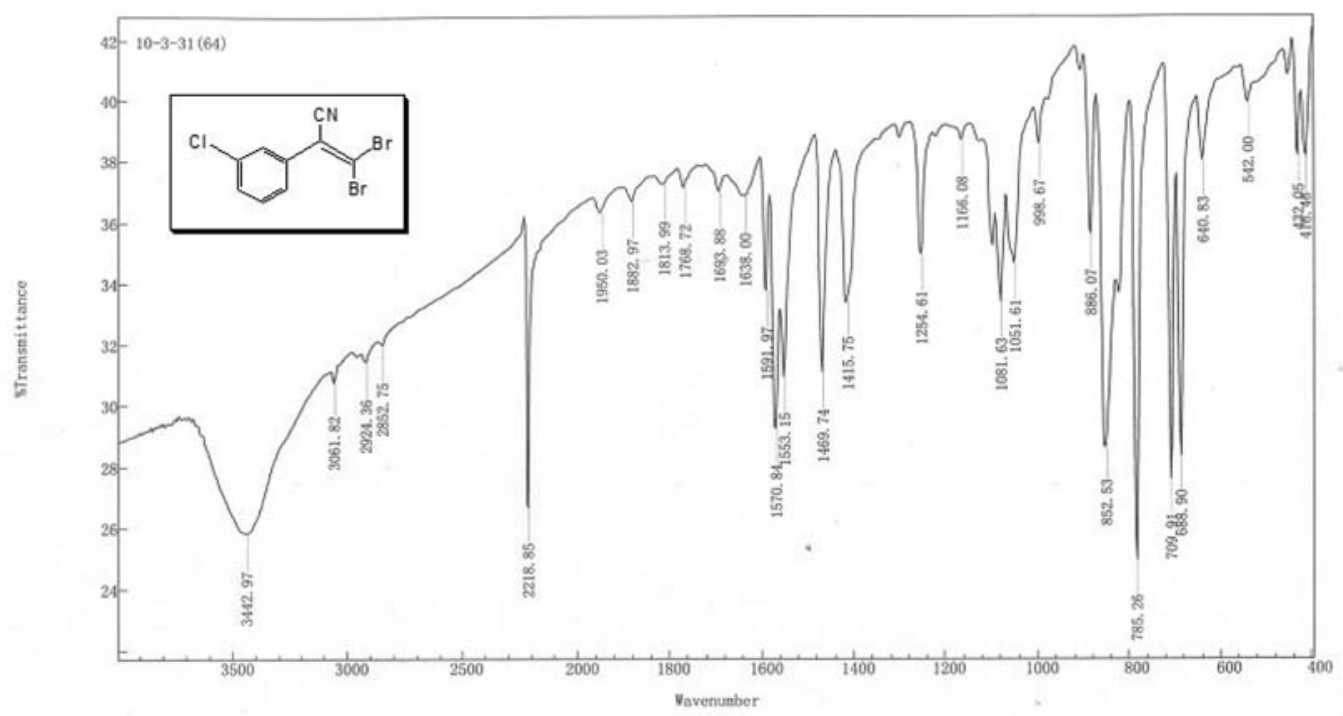

Figure S49. IR spectrum of 2-(3-chlorophenyl)-3,3-dibromoacrylonitrile (2c). 


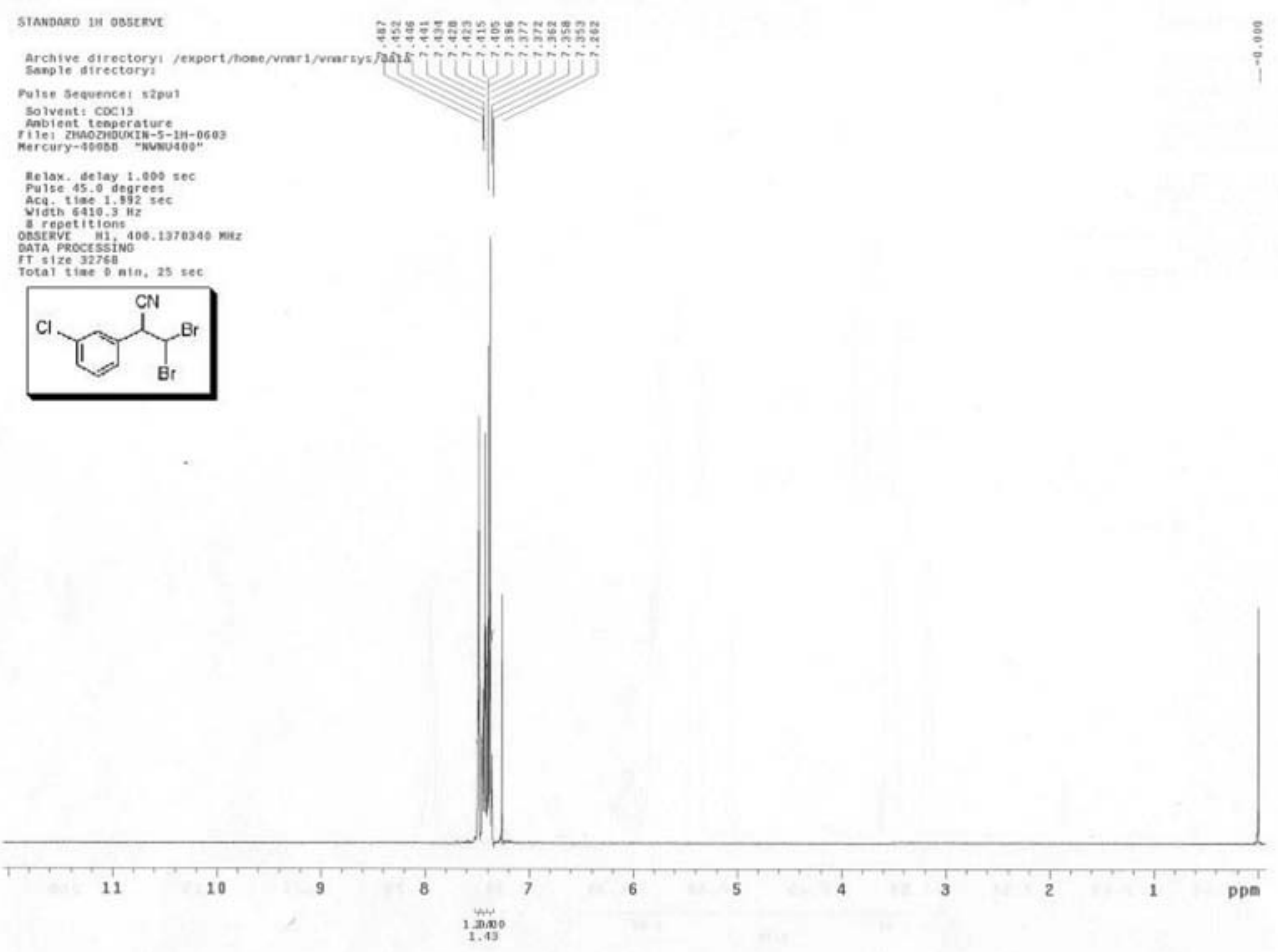

Figure S50. ${ }^{1} \mathrm{H}$ NMR spectrum of 2-(3-chlorophenyl)-3,3-dibromoacrylonitrile (2c).
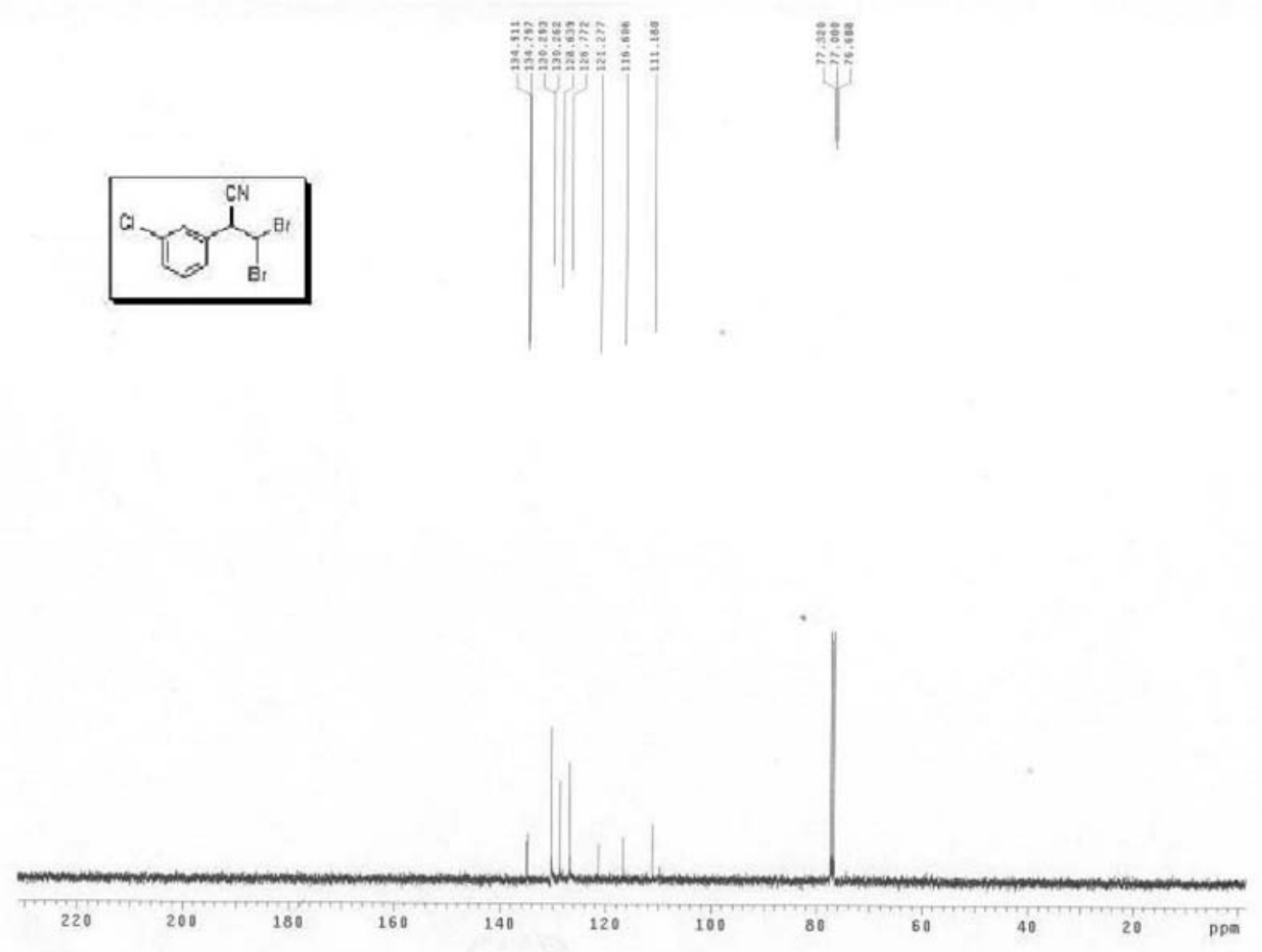

Figure S51. ${ }^{13} \mathrm{C}$ NMR spectrum of 2-(3-chlorophenyl)-3,3-dibromoacrylonitrile (2c). 
2-(4-Chlorophenyl)-3,3-dibromoacrylonitrile (2d)

IR (KBr) $v_{\max } / \mathrm{cm}^{-1}: 2922,2213,1590,1482,1396,1095.831 ;{ }^{1} \mathrm{H}$ NMR $\left(400 \mathrm{MHz}, \mathrm{CDCl}_{3}\right): \delta 7.43(\mathrm{~s}, 4 \mathrm{H}, \mathrm{Ph}-\mathrm{H}) ;{ }^{13} \mathrm{C}$ NMR (CDCl 3 , $100 \mathrm{MHz}): \delta 136.2,131.6,129.9,129.2,121.5,116.7,110.4$.

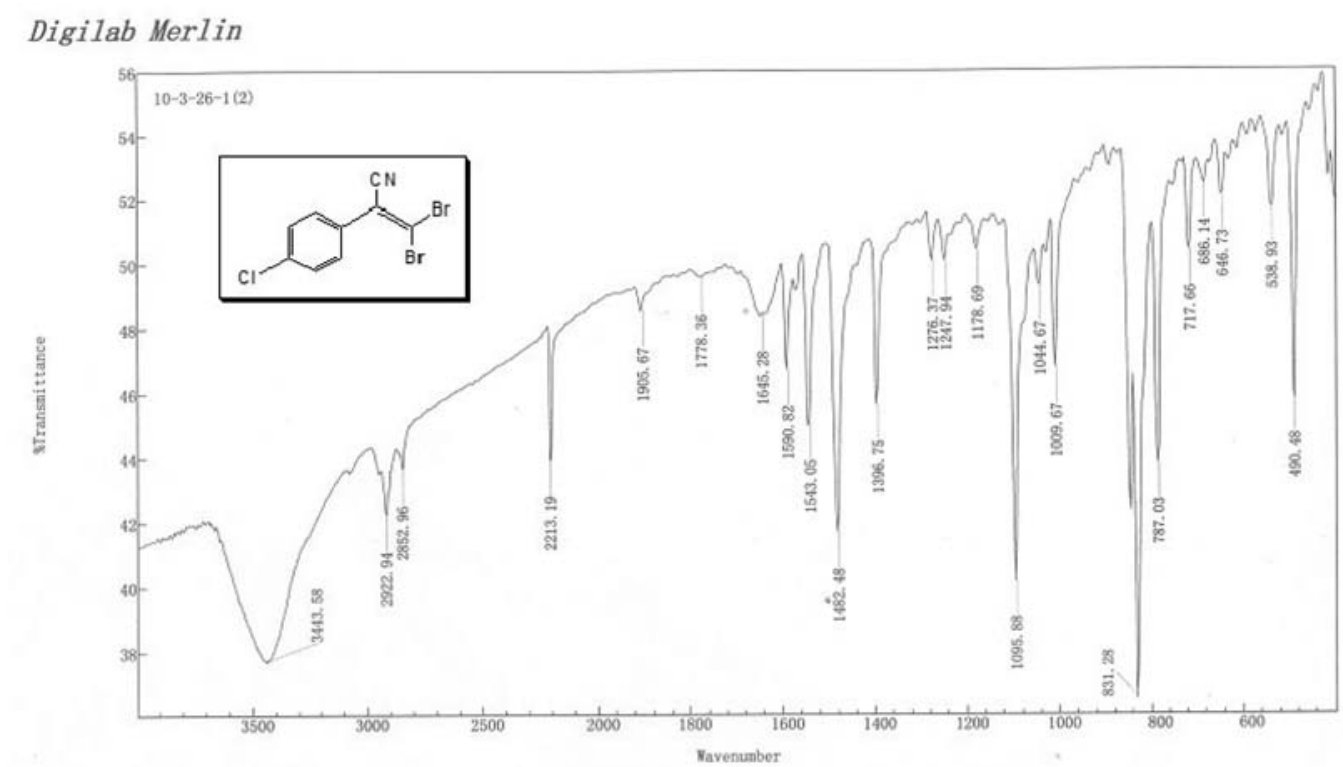

Figure S52. IR spectrum of 2-(4-chlorophenyl)-3,3-dibromoacrylonitrile (2d).
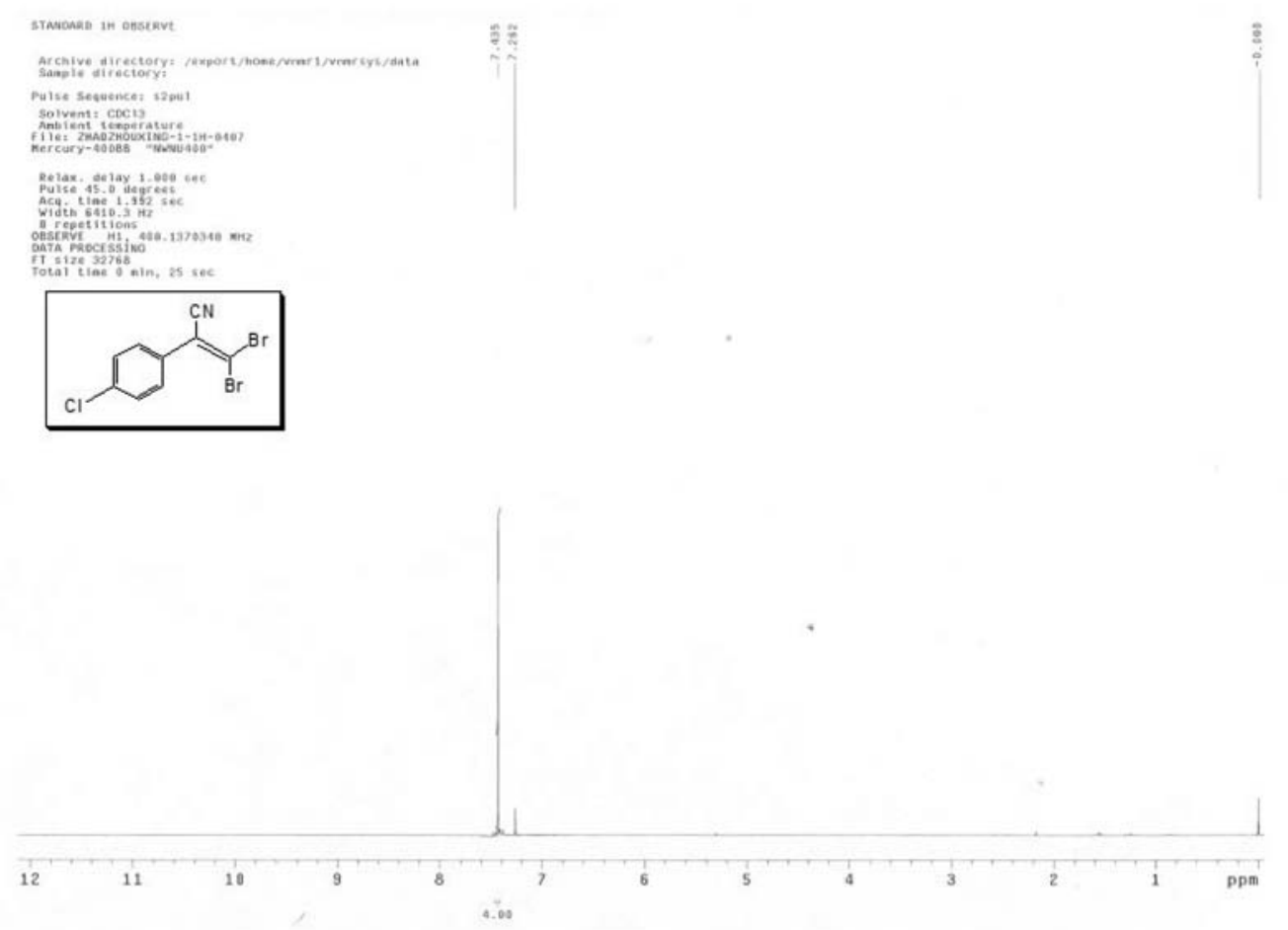

Figure S53. ${ }^{1} \mathrm{H}$ NMR spectrum of 2-(4-chlorophenyl)-3,3-dibromoacrylonitrile (2d). 

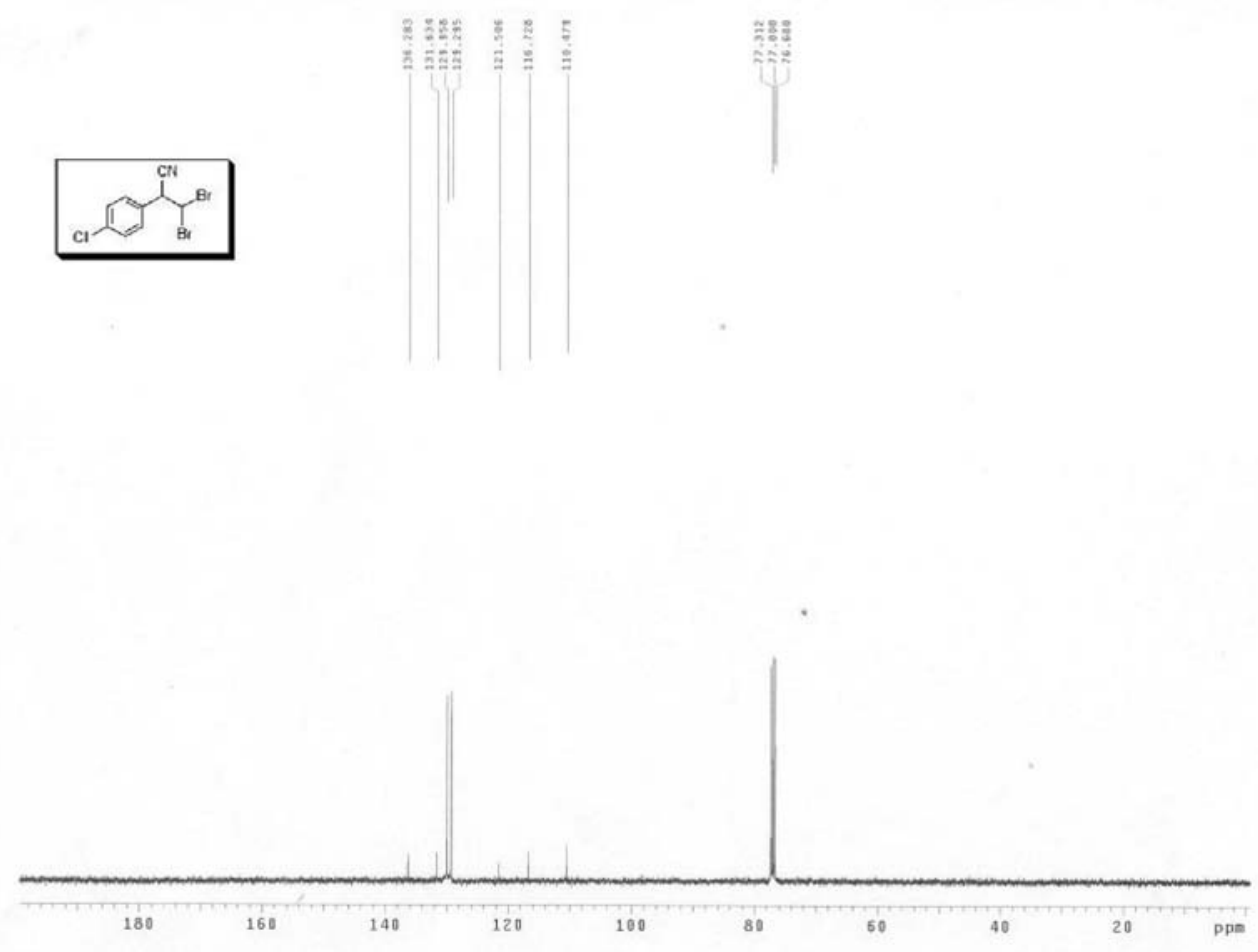

Figure S54. ${ }^{13} \mathrm{C}$ NMR spectrum of 2-(4-chlorophenyl)-3,3-dibromoacrylonitrile (2d).

2-(2-Methylphenyl)-3,3-dibromoacrylonitrile (2e)

IR (KBr) $v_{\max } / \mathrm{cm}^{-1}: 3062,2920,2214,1546,1453,1251,852,735 ;{ }^{1} \mathrm{H}$ NMR $\left(\mathrm{CDCl}_{3}, 400 \mathrm{MHz}\right): \delta 7.37-7.18(\mathrm{~m}, 4 \mathrm{H}$, $\mathrm{Ar}-\mathrm{H}), 2.33$ (s, $\left.3 \mathrm{H}, \mathrm{CH}_{3}\right) ;{ }^{13} \mathrm{C} \mathrm{NMR}\left(\mathrm{CDCl}_{3}, 100 \mathrm{MHz}\right): \delta 136.1,133.3,130.8,130.2,128.8,126.6,122.4,116.1,111.9,19.3$.

\section{Digilab Merlin}

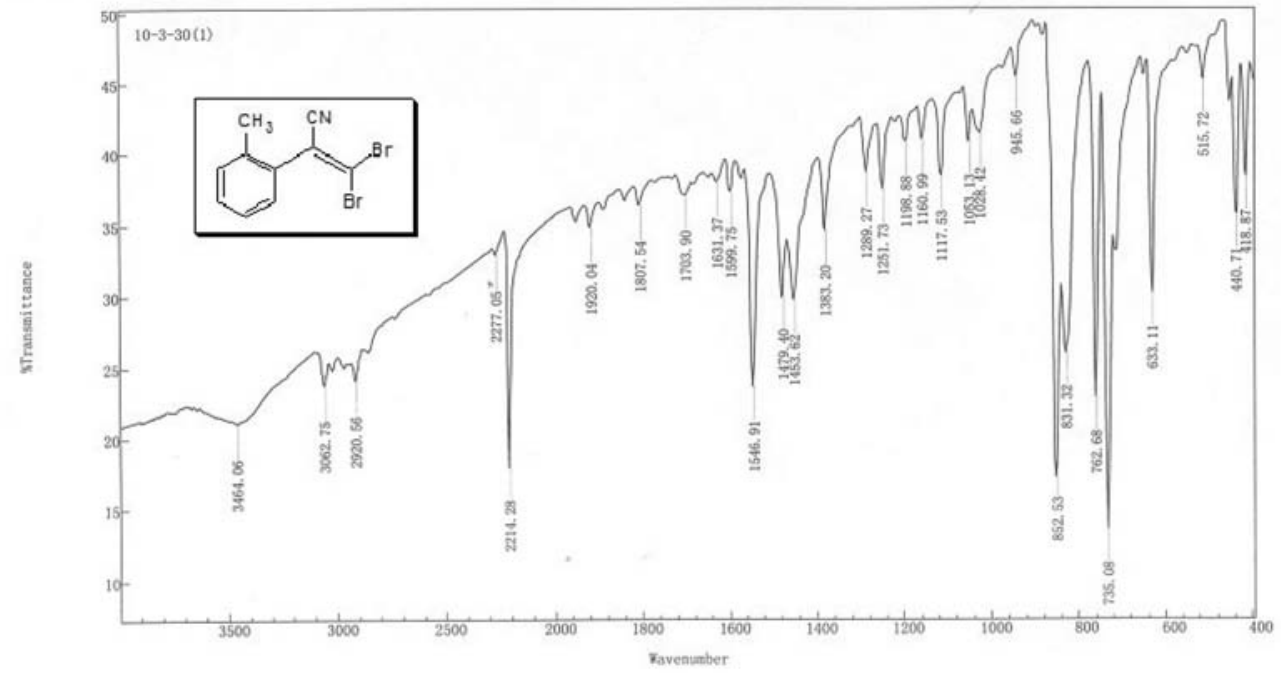

Figure S55. IR spectrum of 2-(2-methylphenyl)-3,3-dibromoacrylonitrile (2e). 


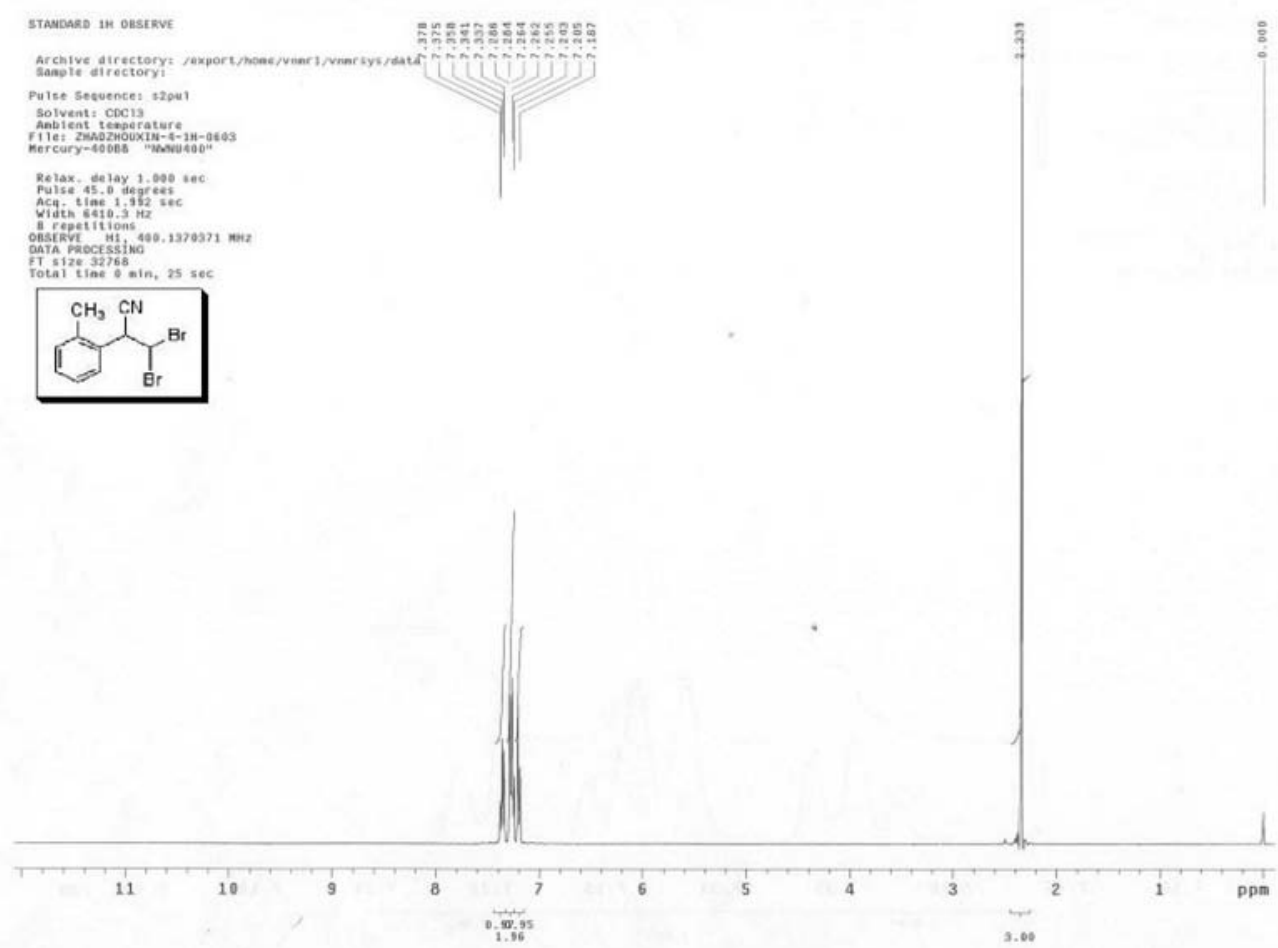

Figure S56. ${ }^{1} \mathrm{H}$ NMR spectrum of 2-(2-methylphenyl)-3,3-dibromoacrylonitrile (2e).

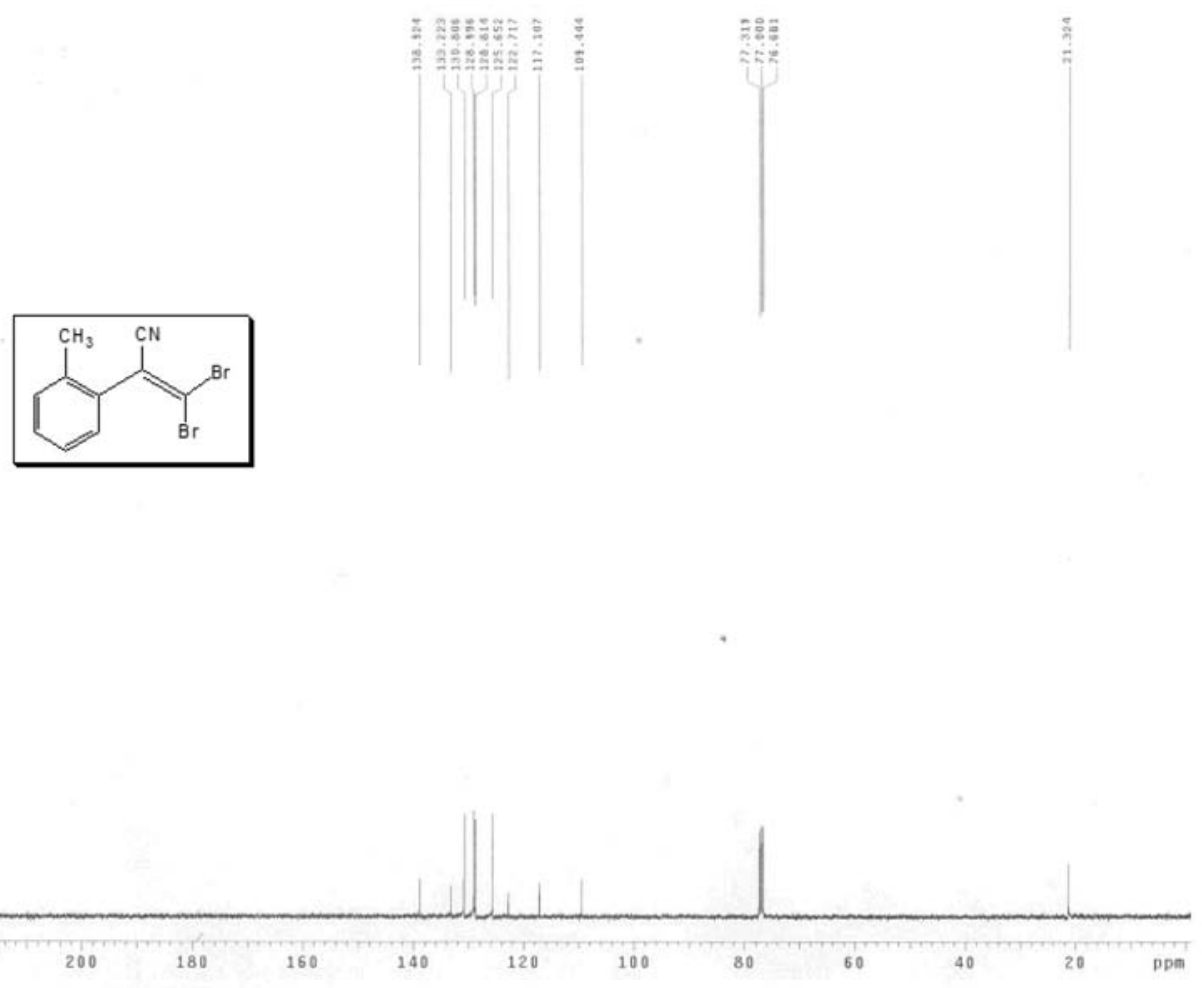

Figure S57. ${ }^{13} \mathrm{C}$ NMR spectrum of 2-(2-methylphenyl)-3,3-dibromoacrylonitrile (2e). 
2-(3-Methylphenyl)-3,3-dibromoacrylonitrile (2f)

IR (KBr) $v_{\max } / \mathrm{cm}^{-1}: 3018,2921,2214,1600,1554,1095,850,789 ;{ }^{1} \mathrm{H}$ NMR $\left(400 \mathrm{MHz}, \mathrm{CDCl}_{3}\right): \delta$ 7.34-7.24 (m, 4H, Ar-H), 2.39 (s, $\left.3 \mathrm{H}, \mathrm{CH}_{3}\right) ;{ }^{13} \mathrm{C} \mathrm{NMR}\left(\mathrm{CDCl}_{3}, 100 \mathrm{MHz}\right): \delta 138.8,133.2,130.8,128.9,128.8,125.6,122.7,117.1,109.4,21.3$.

Digilab Merlin

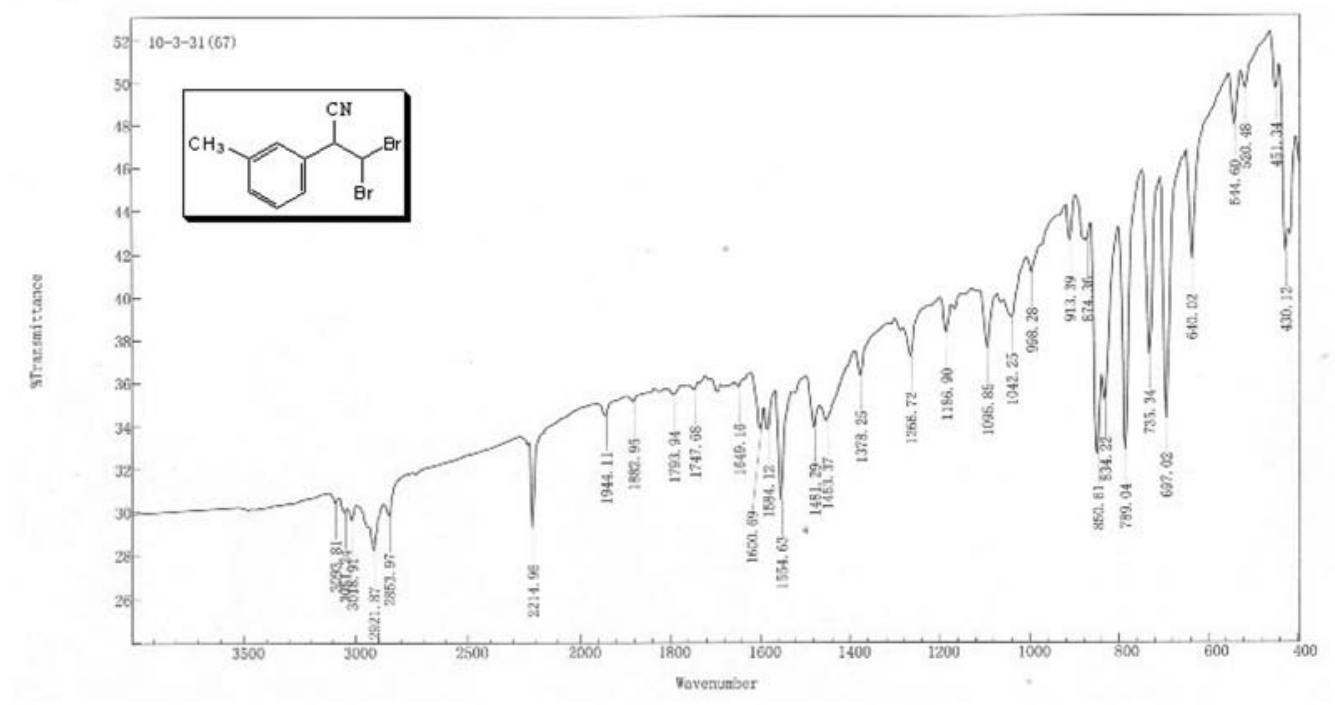

Figure S58. IR spectrum of 2-(3-methylphenyl)-3,3-dibromoacrylonitrile (2f).

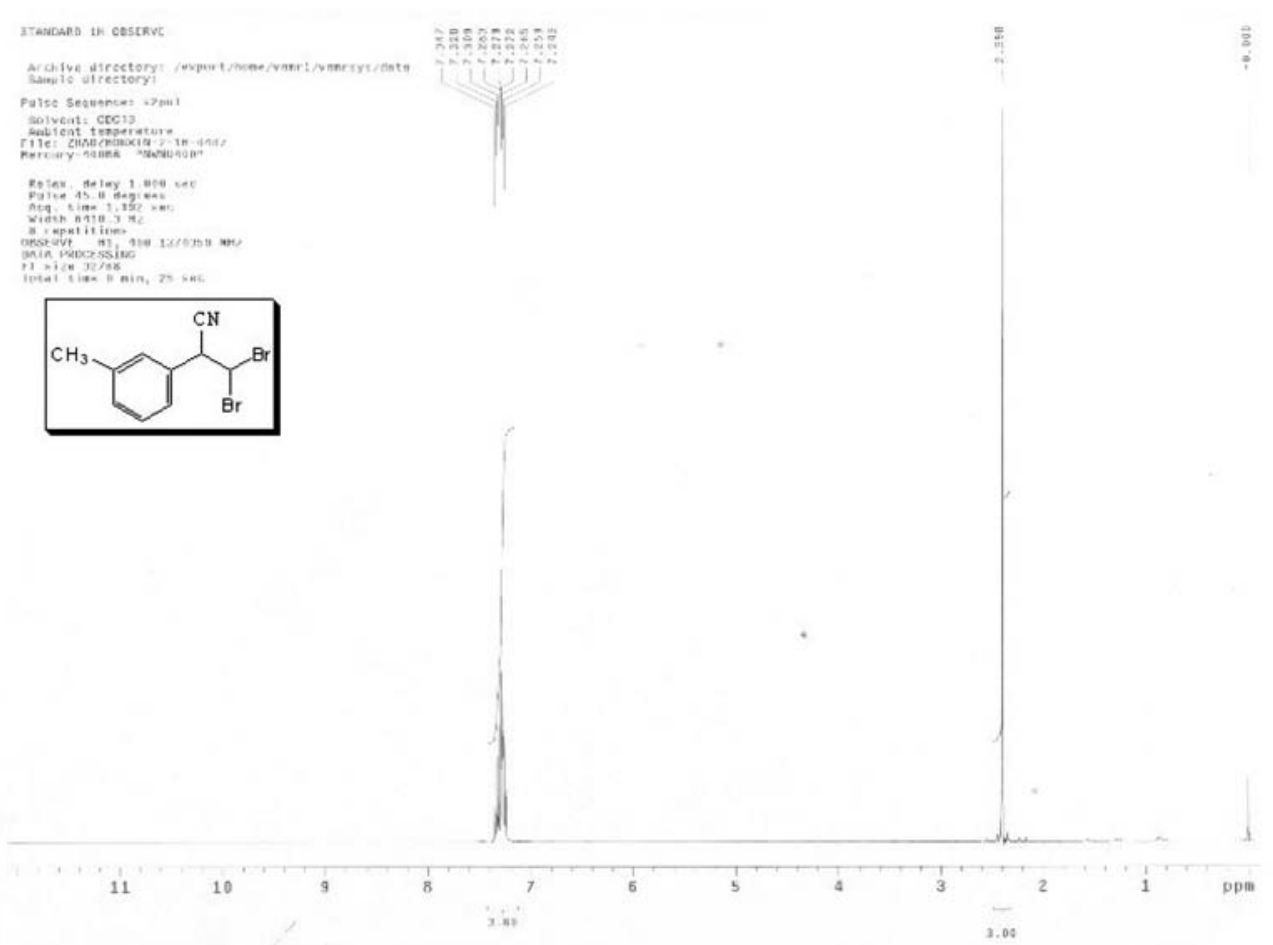

Figure S59. ${ }^{1} \mathrm{H}$ NMR spectrum of 2-(3-methylphenyl)-3,3-dibromoacrylonitrile (2f). 

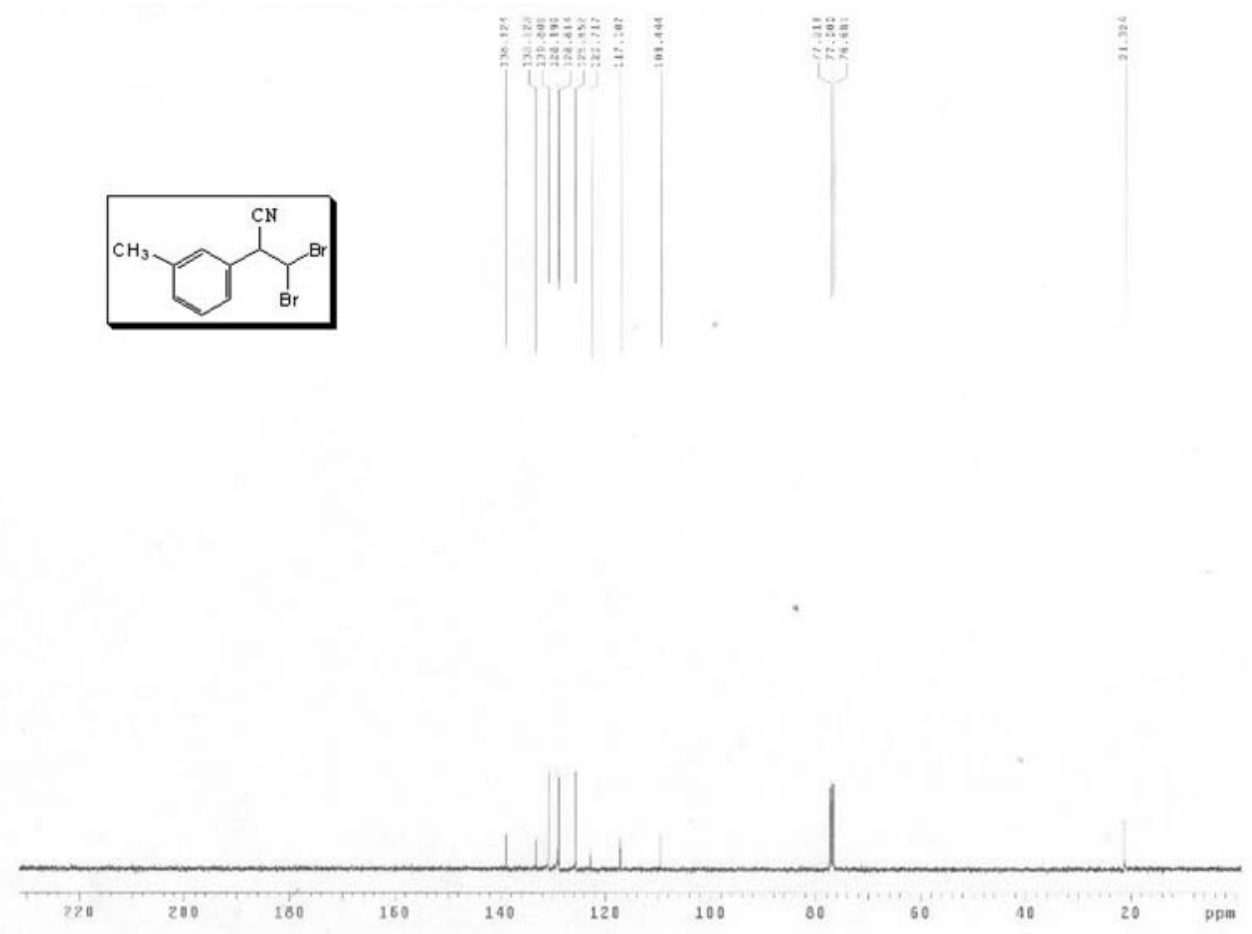

Figure S60. ${ }^{13} \mathrm{C}$ NMRIR spectrum of 2-(3-methylphenyl)-3,3-dibromoacrylonitrile (2f).

\section{2-(4-Methylphenyl)-3,3-dibromoacrylonitrile (2g)}

IR (KBr) $v_{\max } / \mathrm{cm}^{-1}: 3028,2917,2220,1613,1551,1505,1260,849,823,742 ;{ }^{1} \mathrm{H} \mathrm{NMR}\left(\mathrm{CDCl}_{3}, 400 \mathrm{MHz}\right): \delta 7.38(\mathrm{~d}$, $2 \mathrm{H}, J 6.4 \mathrm{~Hz}, \mathrm{Ar}-\mathrm{H}), 7.25$ (d, 2H, $J$ 8.8Hz, Ar-H), 2.38 (s, 3H, $\mathrm{CH}_{3}$ ); $\left.{ }^{13} \mathrm{C} \mathrm{NMR} \mathrm{(CDCl}, 100 \mathrm{MHz}\right): \delta 140.4,130.4,129.6$, 128.4, 122.6, 117.1, 108.9, 21.4.

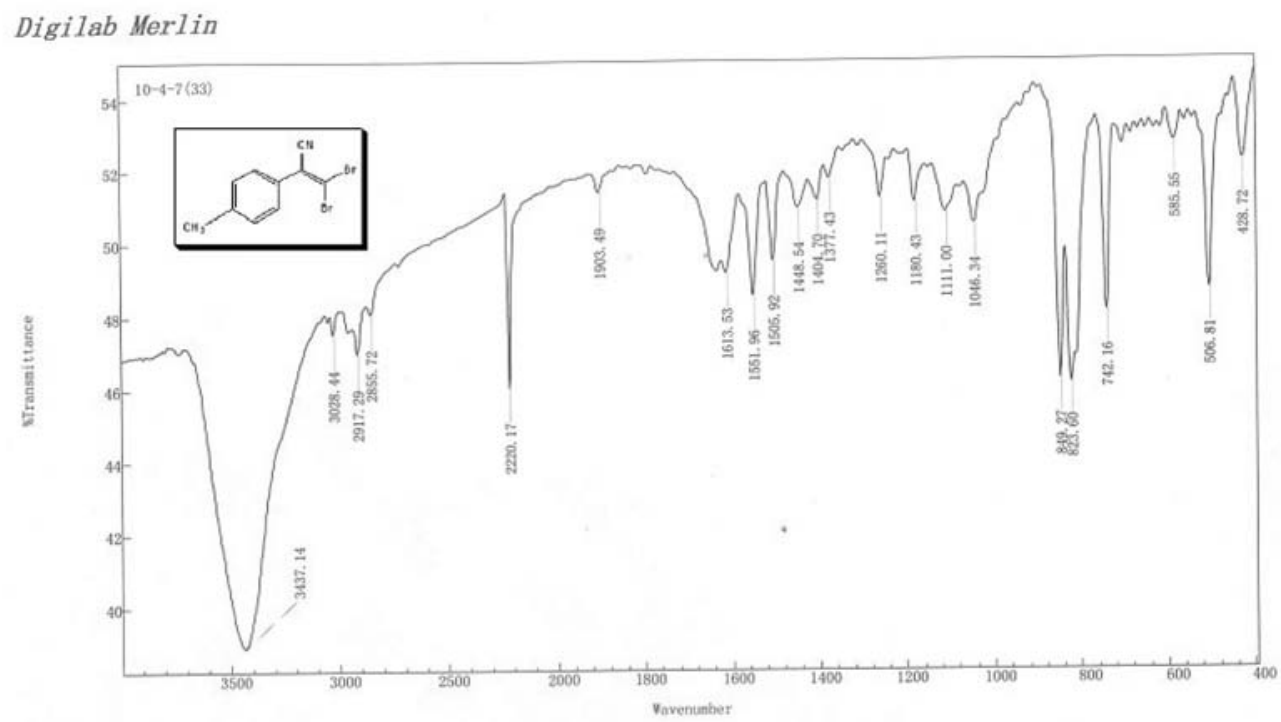

Figure S61. IR spectrum of 2-(4-methylphenyl)-3,3-dibromoacrylonitrile (2g). 


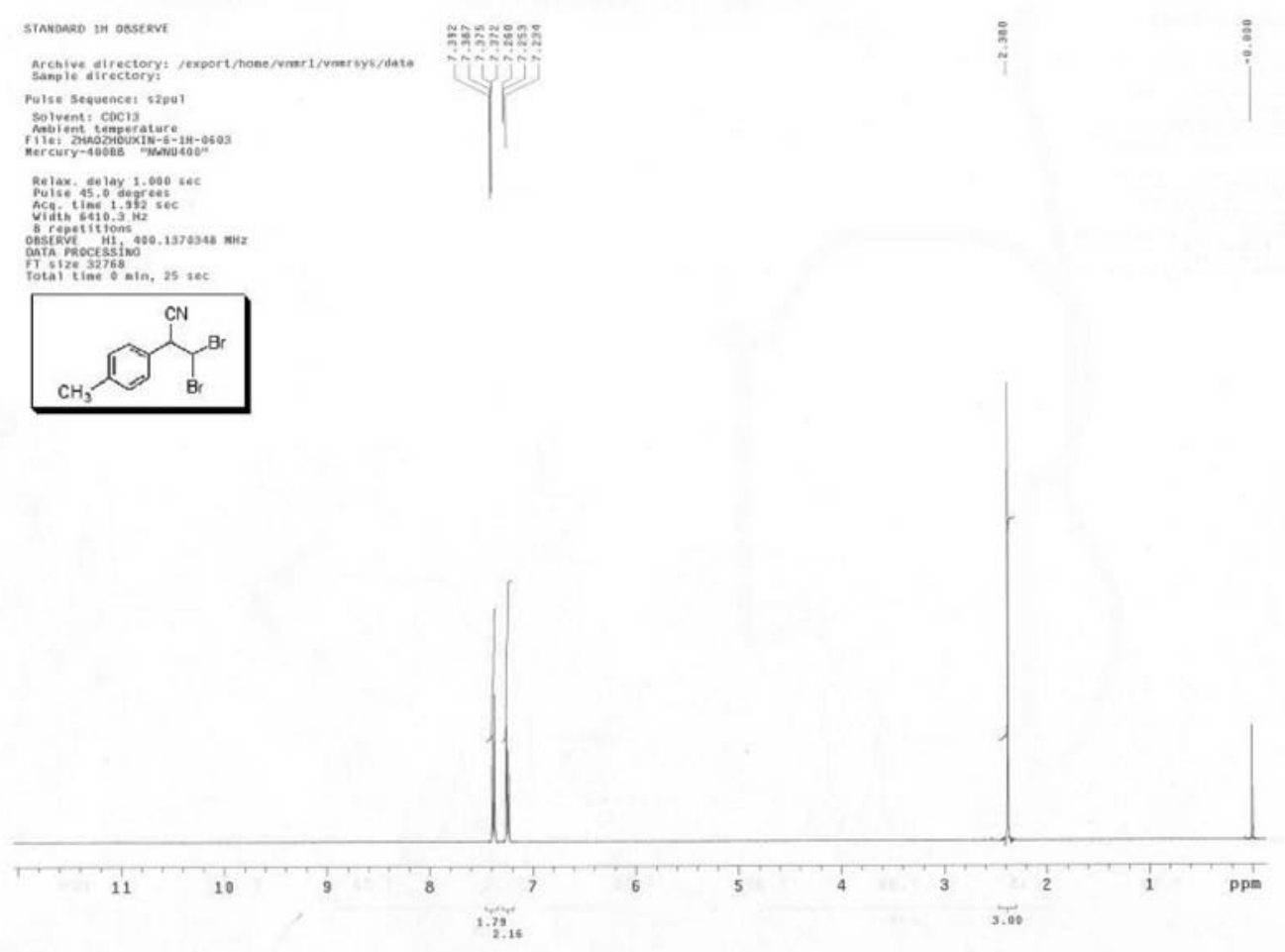

Figure S62. 'H NMR spectrum of 2-(4-methylphenyl)-3,3-dibromoacrylonitrile (2g).
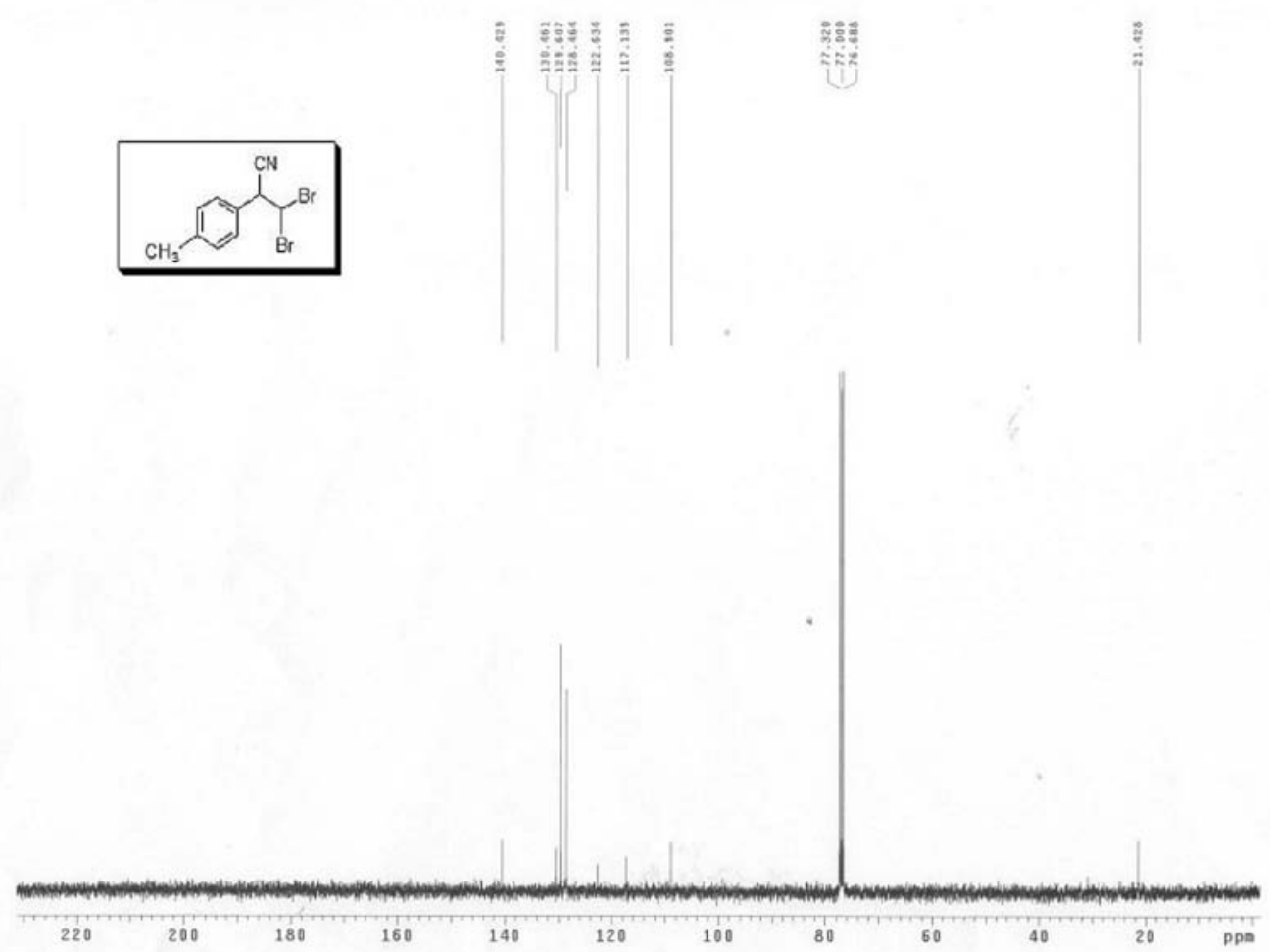

Figure S63. ${ }^{13} \mathrm{C}$ NMR spectrum of 2-(4-methylphenyl)-3,3-dibromoacrylonitrile (2g). 
2-(4-Nitrophenyl)-3,3-dibromoacrylonitrile (2h)

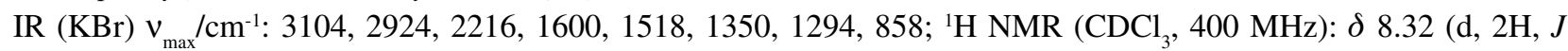

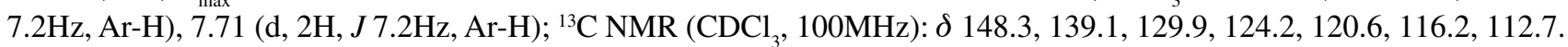

Digilab Merlin

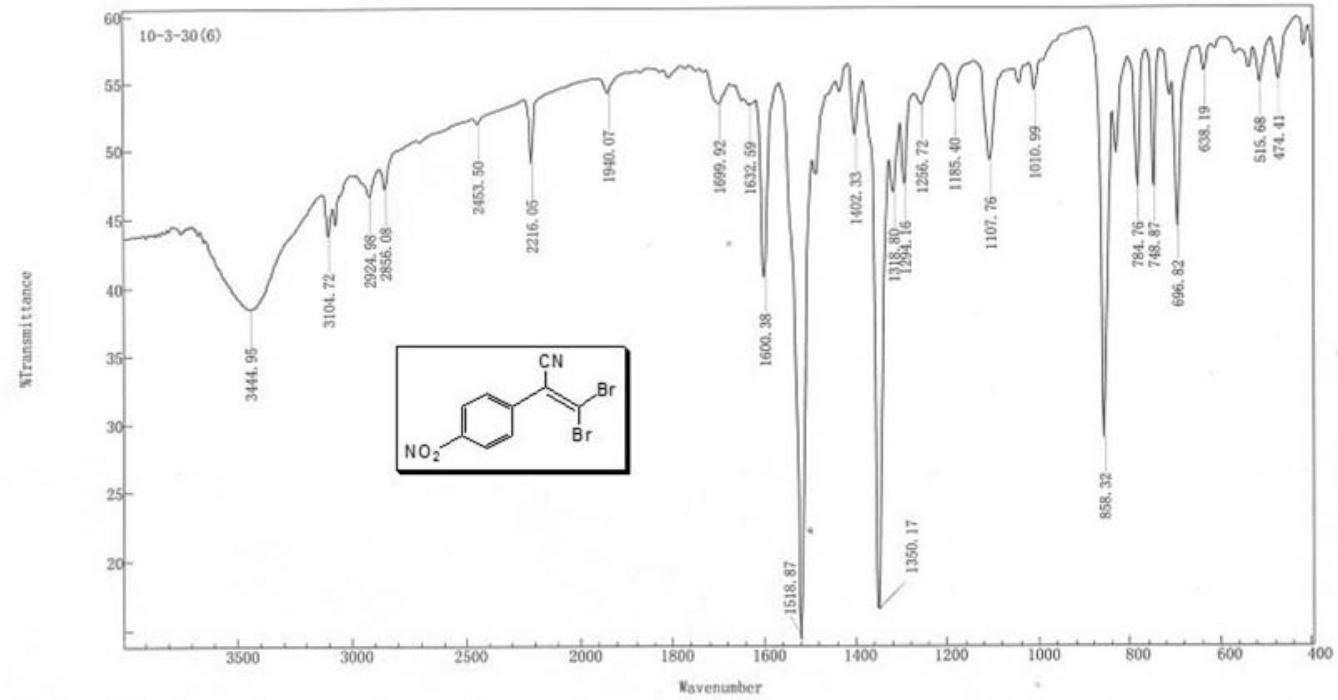

Figure S64. IR spectrum of 2-(4-nitrophenyl)-3,3-dibromoacrylonitrile (2h).

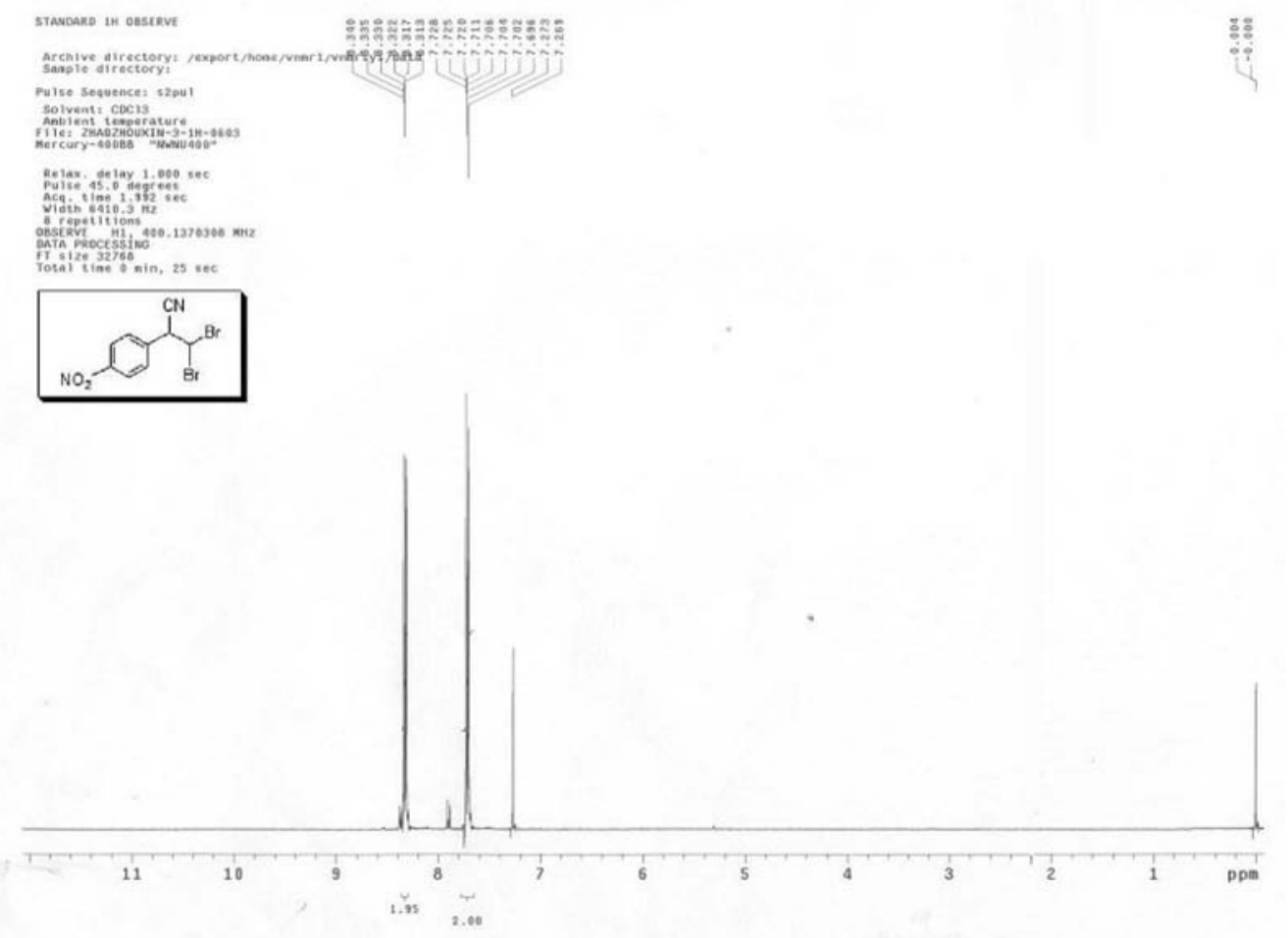

Figure S65. ${ }^{1} \mathrm{H}$ NMR spectrum of 2-(4-nitrophenyl)-3,3-dibromoacrylonitrile (2h). 

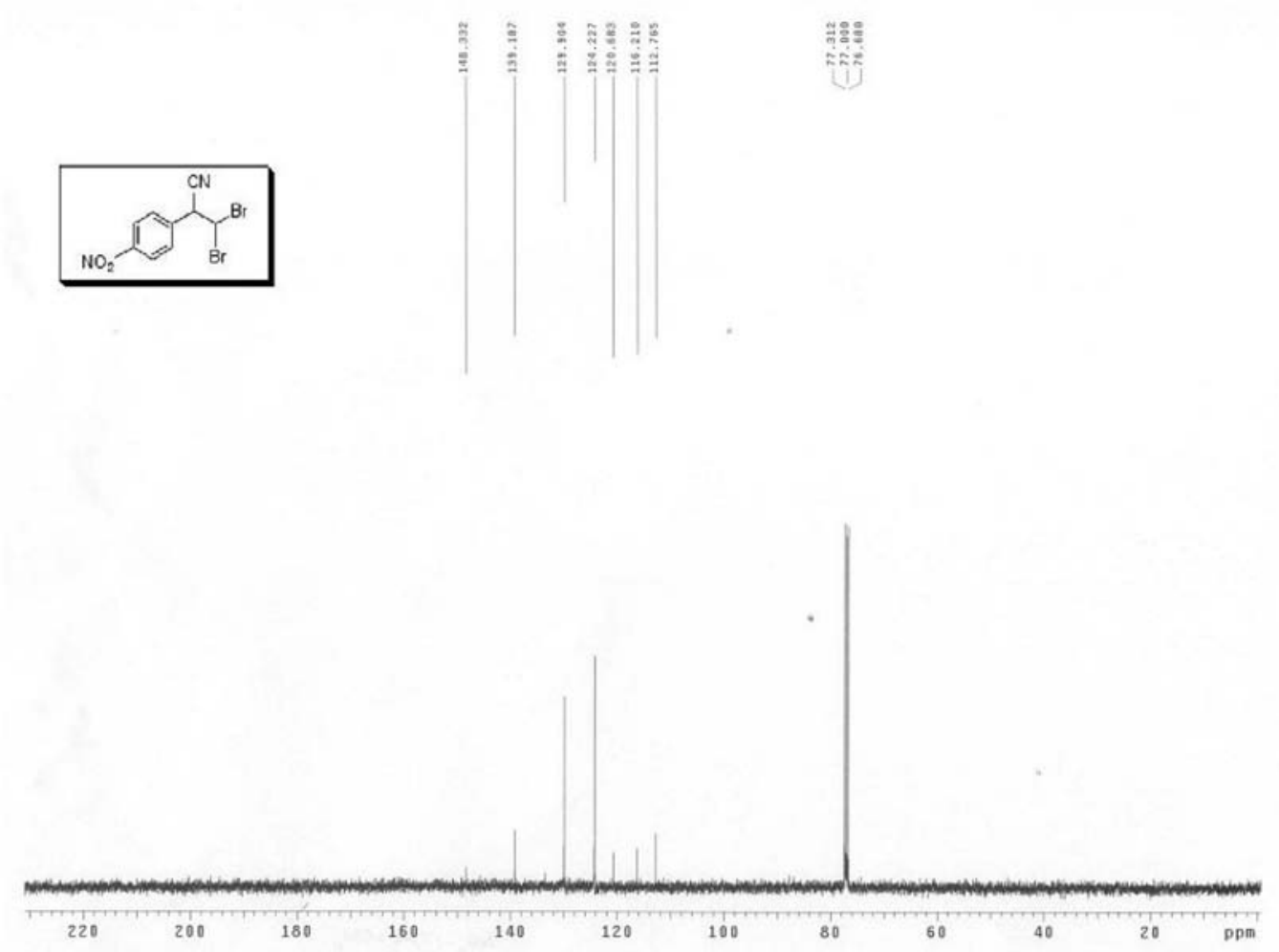

Figure S66. ${ }^{13} \mathrm{C}$ NMR spectrum of 2-(4-nitrophenyl)-3,3-dibromoacrylonitrile (2h).

\section{2-(3,5-Dinitrophenyl)-3,3-dibromoacrylonitrile (2i)}

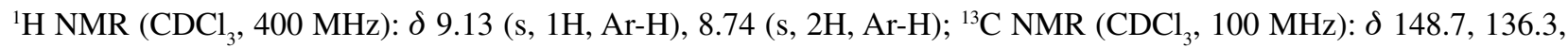
129.0, 128.9, 120.0, 118.4, 115.6; IR (KBr) $v_{\text {max }} / \mathrm{cm}^{-1}: 3094,2983,2224,1627,1543,1344,1279,918,853,729$.

\section{Digilab Merlin}

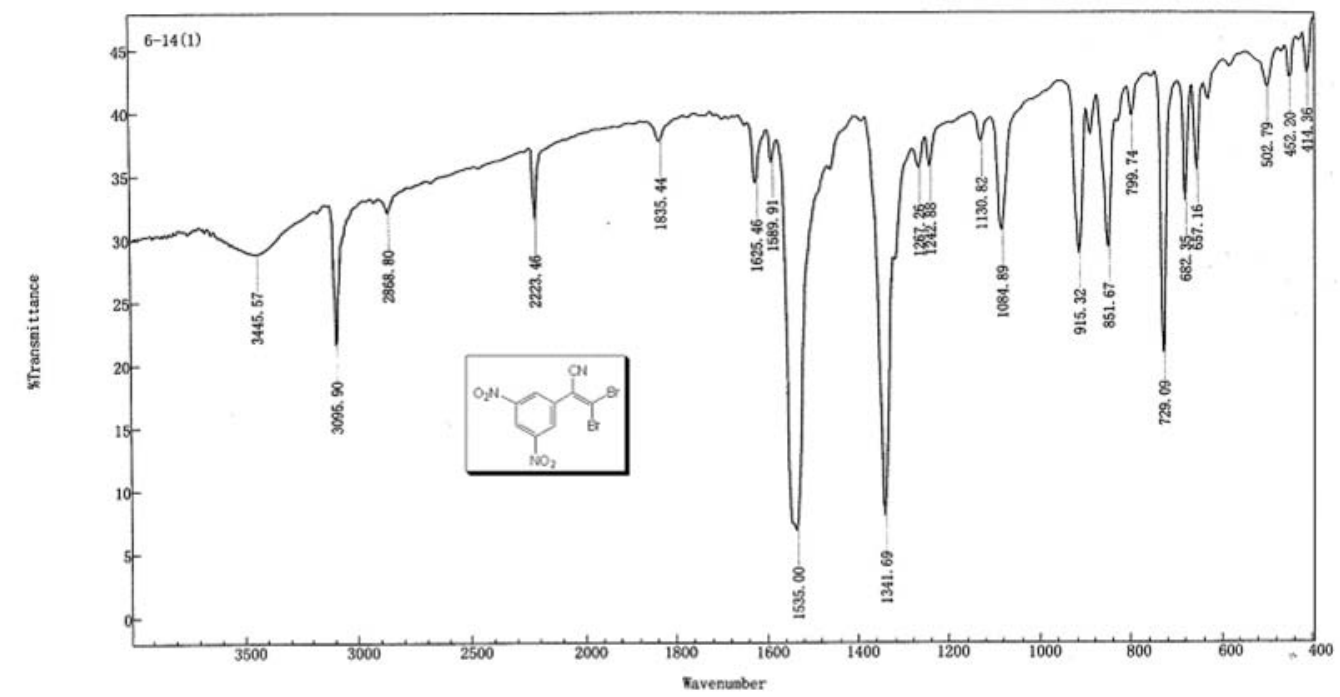

??????????????

Figure S67. IR spectrum of 2-(3,5-dinitrophenyl)-3,3-dibromoacrylonitrile (2i). 


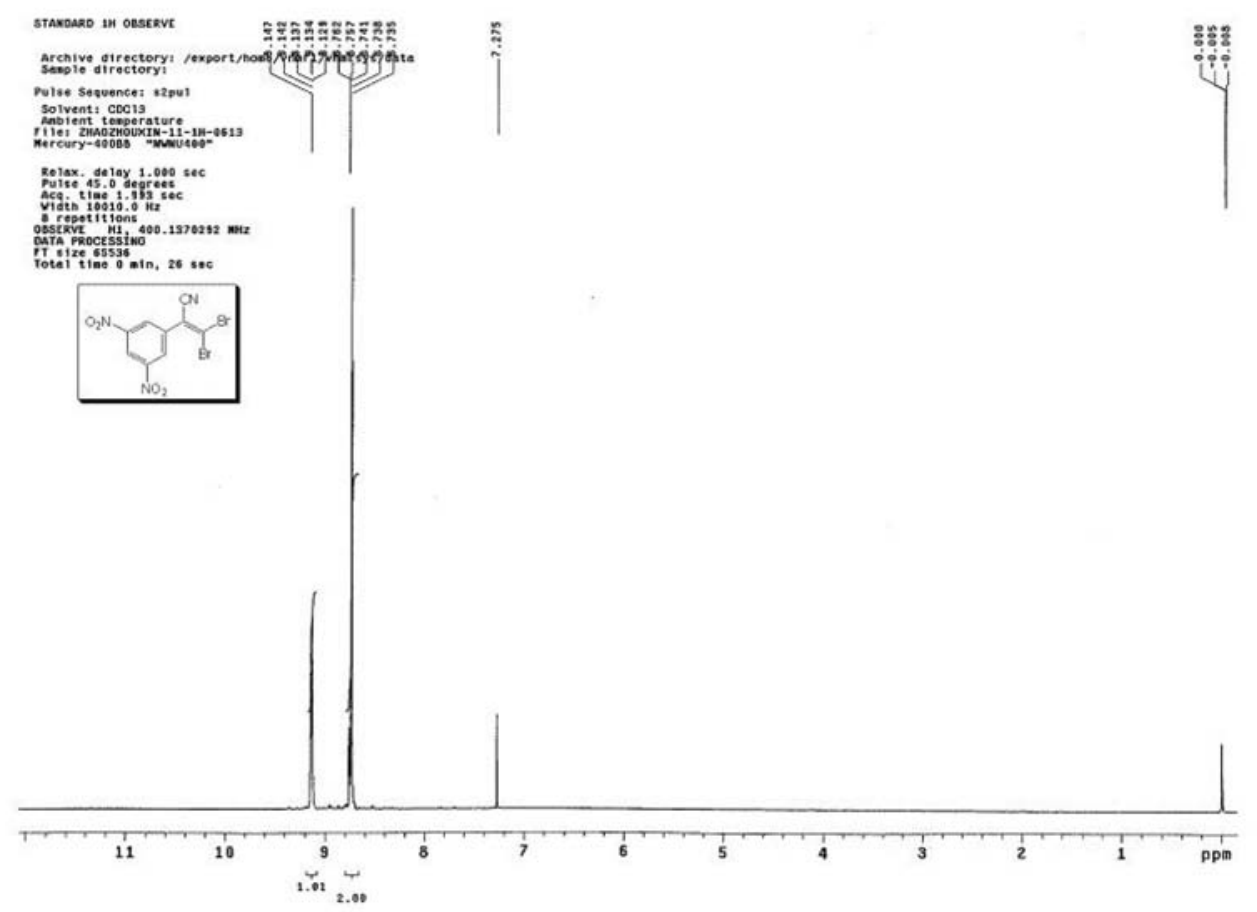

Figure S68. ${ }^{1} \mathrm{H}$ NMR spectrum of 2-(3,5-dinitrophenyl)-3,3-dibromoacrylonitrile (2i).
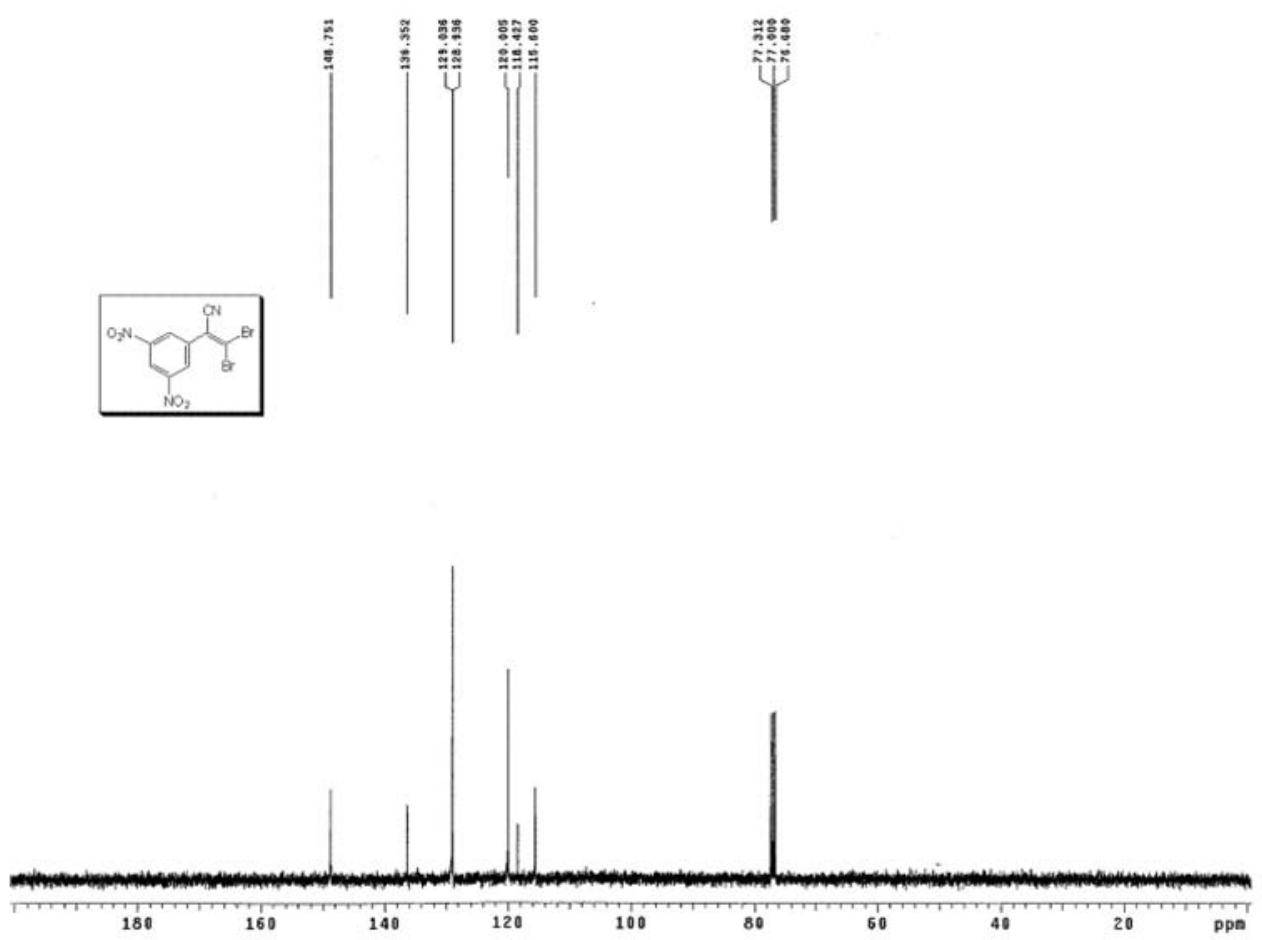

Figure S69. ${ }^{13} \mathrm{C}$ NMR spectrum of 2-(3,5-dinitrophenyl)-3,3-dibromoacrylonitrile (2i). 
2-(3-Nitrophenyl)-3,3-dibromoacrylonitrile (2j)

IR (KBr) $v_{\max } / \mathrm{cm}^{-1}: 3088,2924,2218,1615,1531,1346,1255,860,687 ;{ }^{1} \mathrm{H} \mathrm{NMR}\left(\mathrm{CDCl}_{3}, 400 \mathrm{MHz}\right): \delta 8.41(\mathrm{~s}, 1 \mathrm{H}$, Ar-H), 8.33 (d, $1 \mathrm{H}, J 8.4 \mathrm{~Hz}, \mathrm{Ar}-\mathrm{H}), 7.84$ (d, $1 \mathrm{H}, J 8.4 \mathrm{~Hz}, \mathrm{Ar}-\mathrm{H}), 7.69$ (t, $1 \mathrm{H}, J 8.4 \mathrm{~Hz}, \mathrm{Ar}-\mathrm{H}) ;{ }^{13} \mathrm{C} \mathrm{NMR}\left(\mathrm{CDCl}_{3}, 100 \mathrm{MHz}\right)$ : $\delta 148.3,134.7,134.5,130.3,124.8,123.8,120.3,116.2,112.8$.

\section{Digilab Merlin}

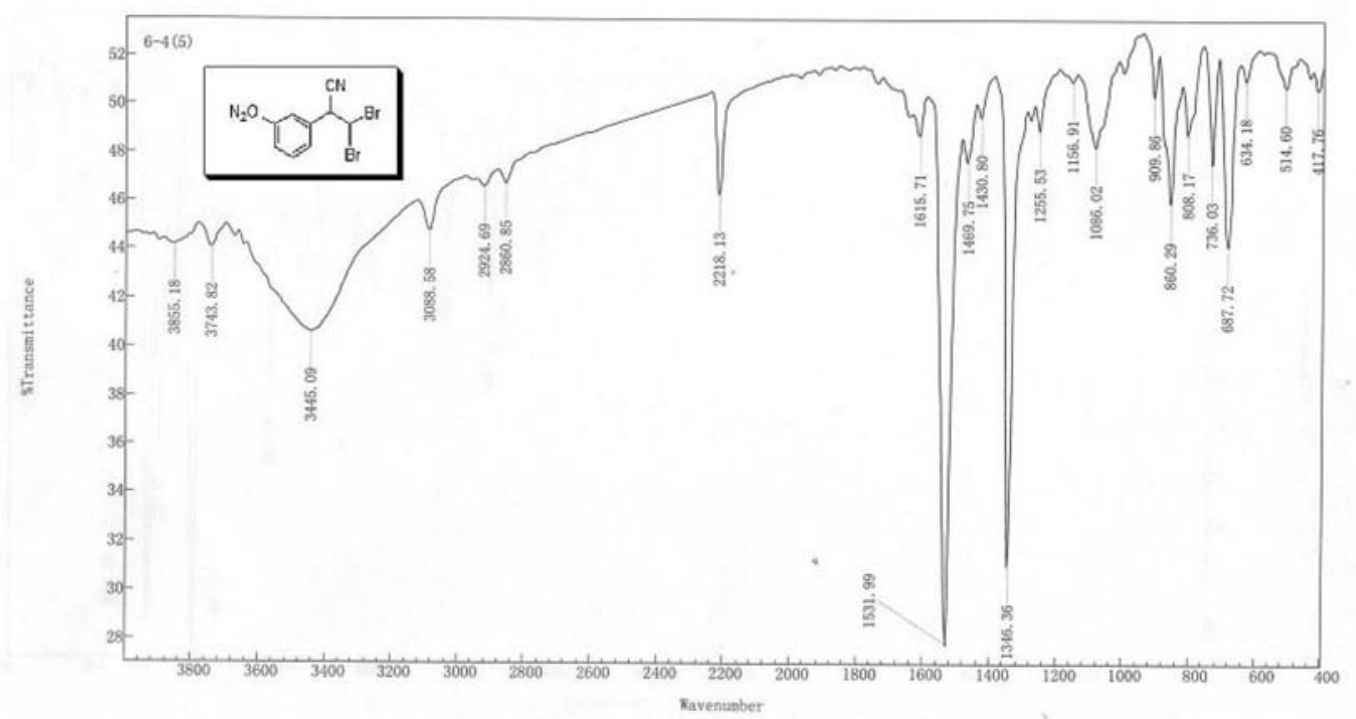

Figure S70. IR spectrum of 2-(3-nitrophenyl)-3,3-dibromoacrylonitrile (2j).

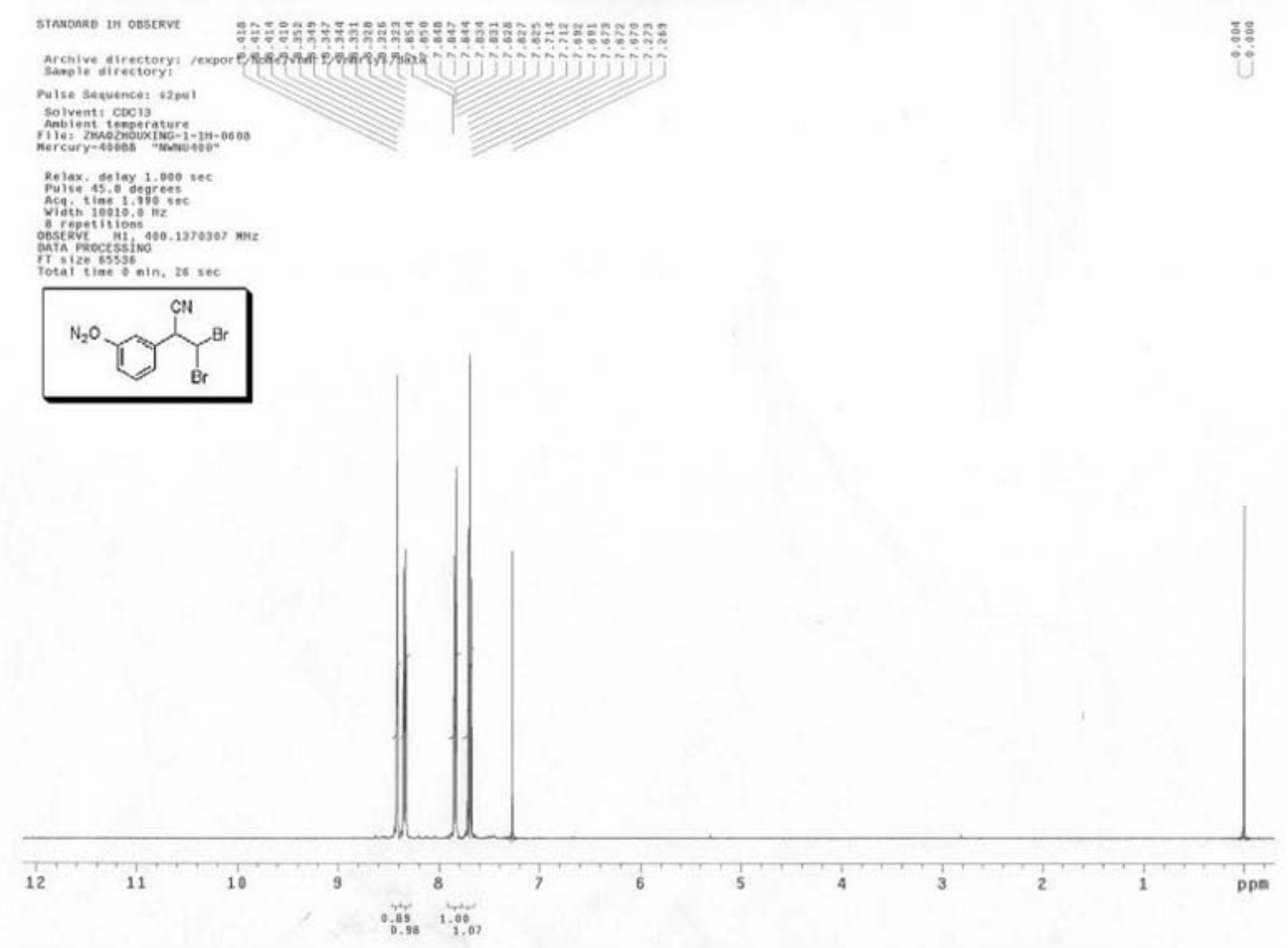

Figure S71. ${ }^{1} \mathrm{H}$ NMR spectrum of 2-(3-nitrophenyl)-3,3-dibromoacrylonitrile (2j). 

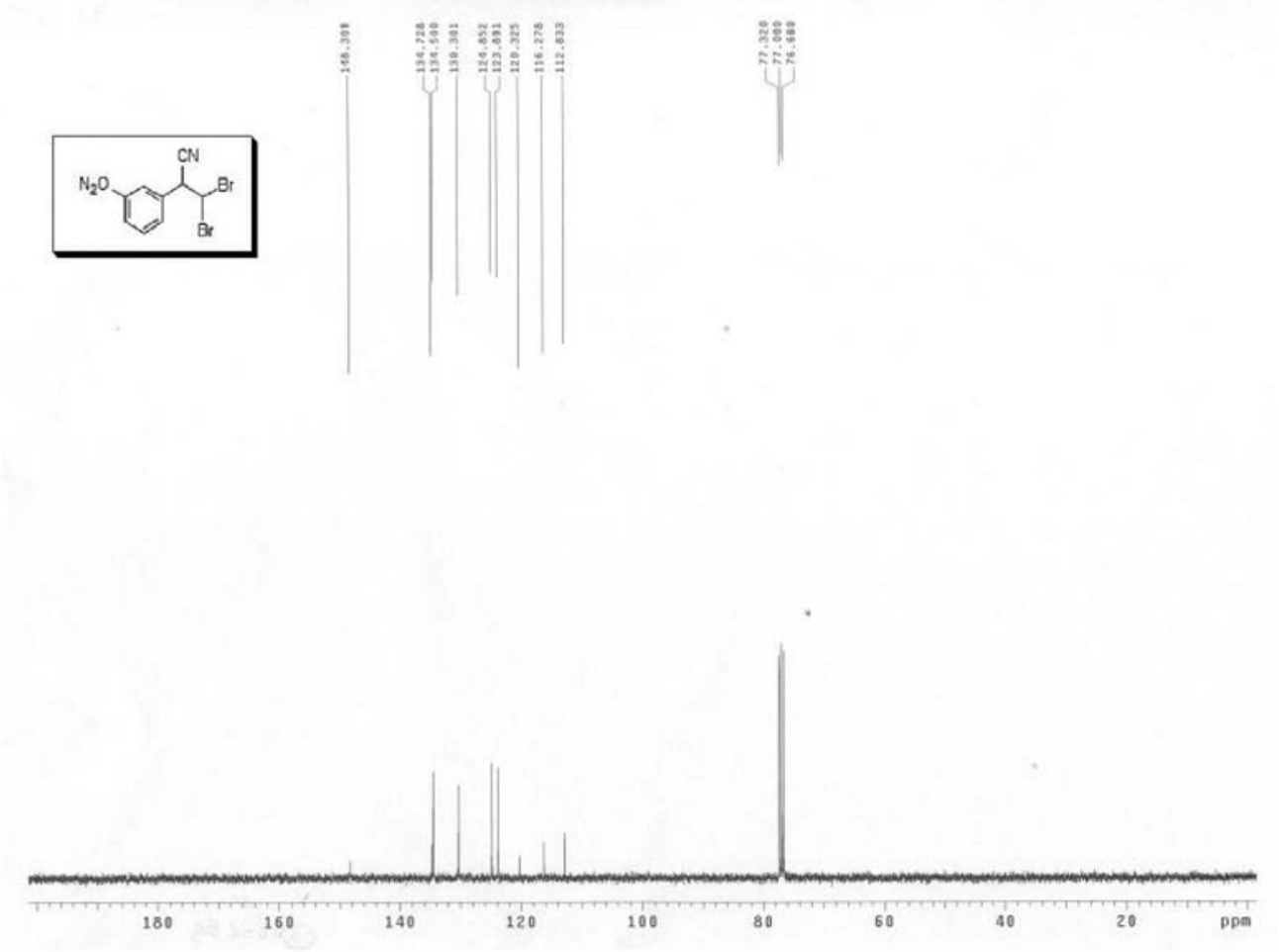

Figure S72. ${ }^{13} \mathrm{C}$ NMR spectrum of 2-(3-nitrophenyl)-3,3-dibromoacrylonitrile (2j).

\section{2-(4--Methoxyphenyl)-3,3-dibromoacrylonitrile (2k)}

IR (KBr) $v_{\text {max }} / \mathrm{cm}^{-1}: 2999,2939,2214,1605,1508,1259,1182,1021,829 .{ }^{1} \mathrm{H}$ NMR $\left(\mathrm{CDCl}_{3}, 400 \mathrm{MHz}\right): \delta 7.44(\mathrm{~d}, 2 \mathrm{H}, J$ $9.2 \mathrm{~Hz}, \mathrm{Ar}-\mathrm{H}), 6.94$ (d, $2 \mathrm{H}, J 9.2 \mathrm{~Hz}, \mathrm{Ar}-\mathrm{H}), 3.84$ (s, 3H, $\left.\mathrm{CH}_{3}\right) .{ }^{13} \mathrm{C} \mathrm{NMR}\left(\mathrm{CDCl}_{3}, 100 \mathrm{MHz}\right): \delta 160.6,130.1,125.4,122.2$, 117.1, 114.2, 108.0, 55.3.

\section{Digilab Merlin}

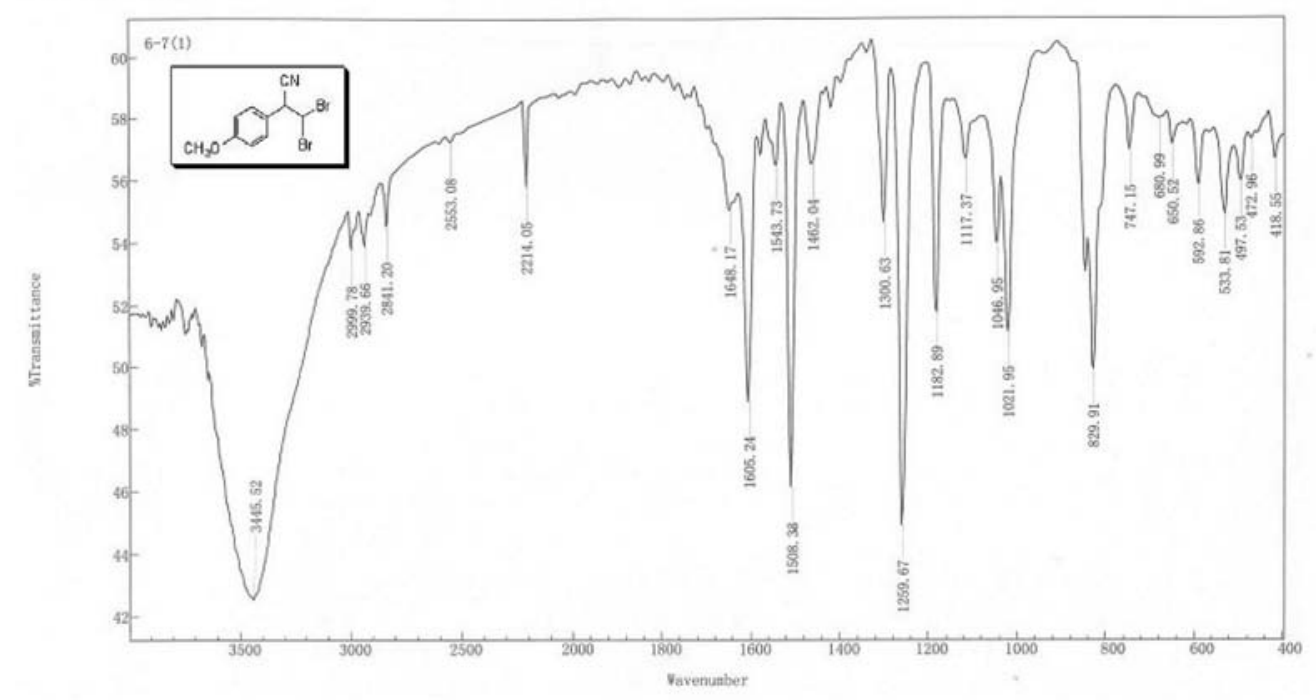

Figure S73. IR spectrum of 2-(4-methoxyphenyl)-3,3-dibromoacrylonitrile (2k). 


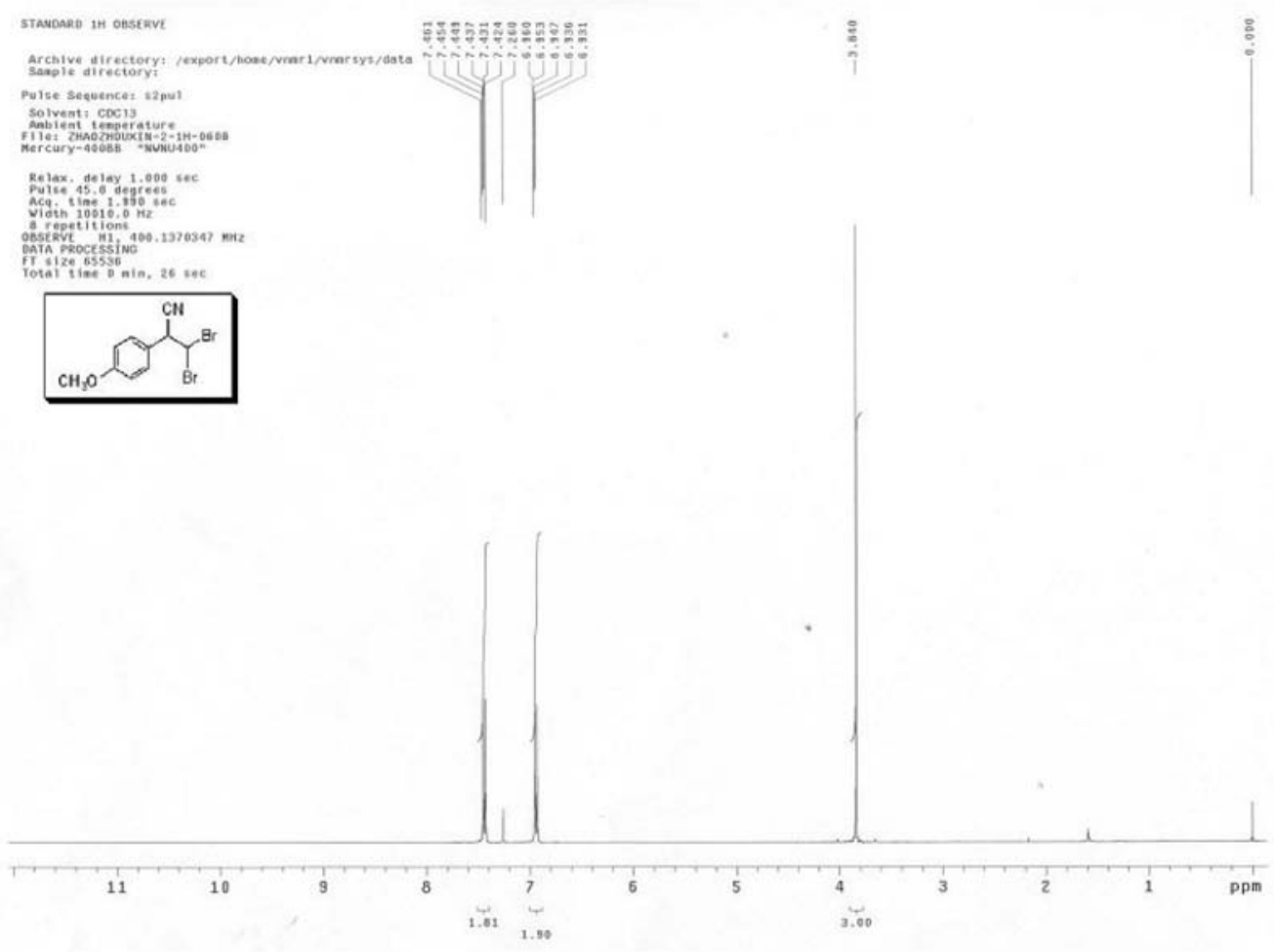

Figure S74. ' H NMR spectrum of 2-(4-methoxyphenyl)-3,3-dibromoacrylonitrile (2k).
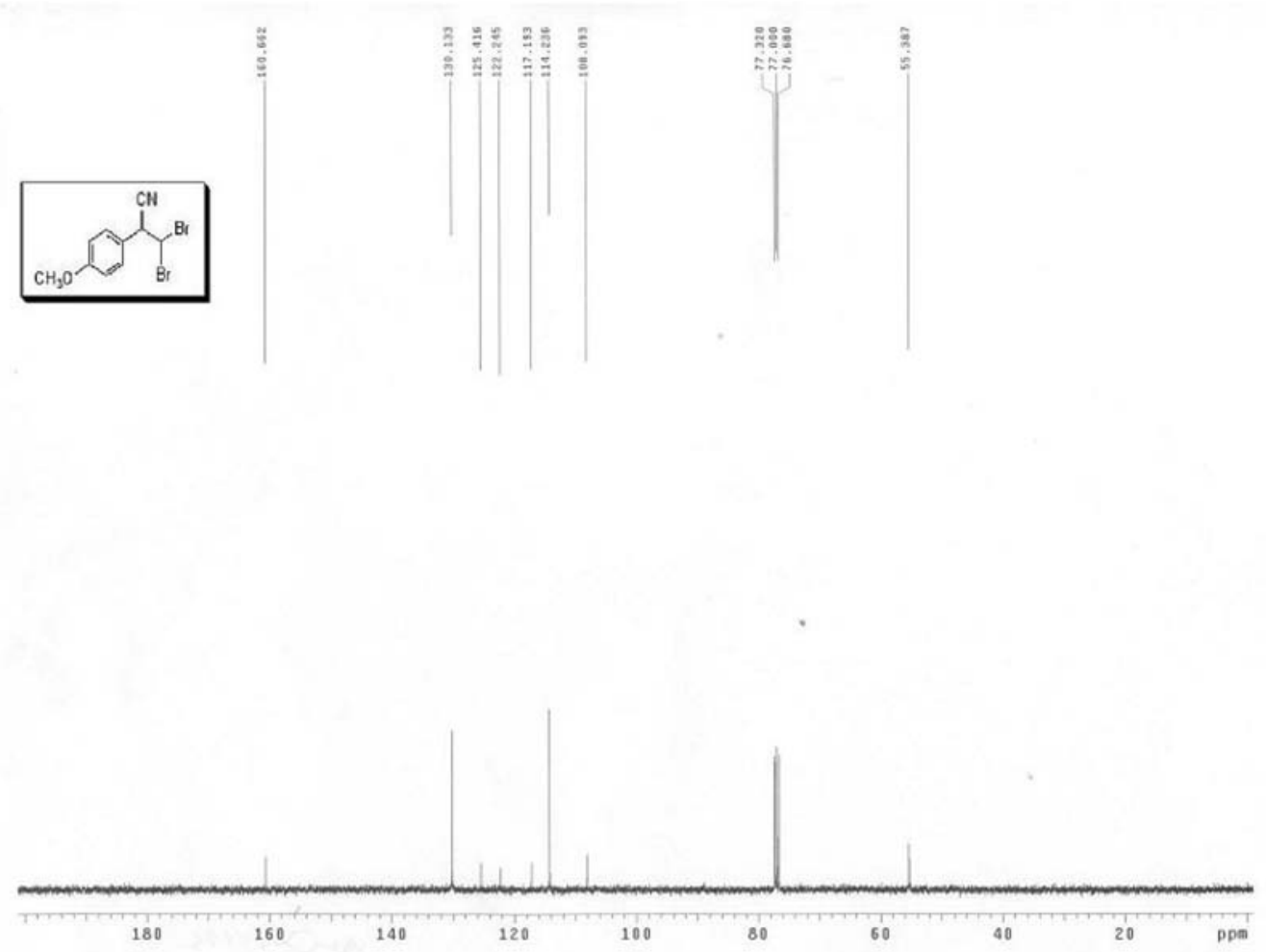

Figure S75. ${ }^{13} \mathrm{C}$ NMR spectrum of 2-(4-methoxyphenyl)-3,3-dibromoacrylonitrile (2k). 
2-(4-Biphenyl)-3,3-dibromoacrylonitrile (2l)

IR (KBr) $v_{\max } / \mathrm{cm}^{-1}: 3063,2922,2223,1600,1479,1400,842,767 ;{ }^{1} \mathrm{H}$ NMR $\left(400 \mathrm{MHz}, \mathrm{CDCl}_{3}\right): \delta 7.74-7.42(\mathrm{~m}, 9 \mathrm{H}$, Ar-H); ${ }^{13} \mathrm{C} \mathrm{NMR}\left(\mathrm{CDCl}_{3}, 100 \mathrm{MHz}\right): \delta$ 145.6, 139.1, 132.5, 132.4, 129.0, 128.6, 127.7, 127.2, 122.1, 118.9, 110.8 .

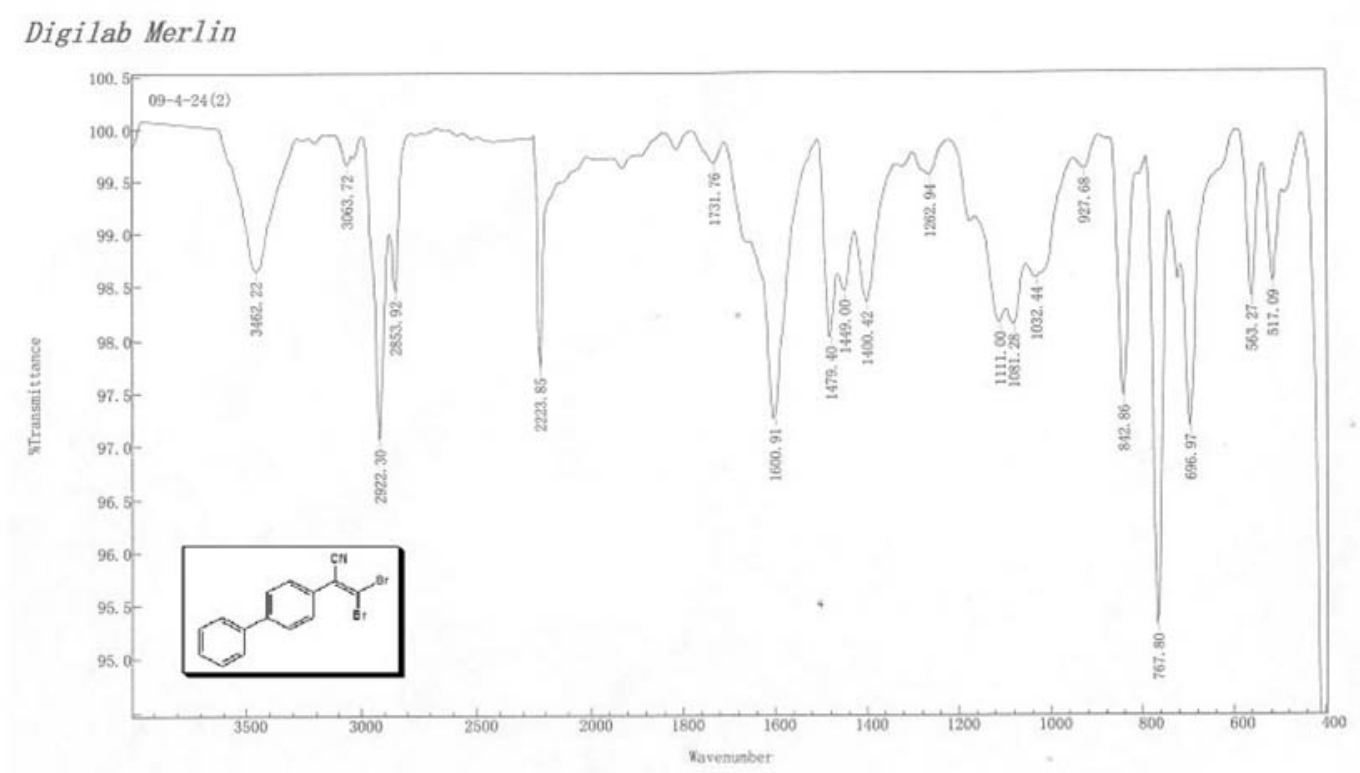

Figure S76. IR spectrum of 2-(4-biphenyl)-3,3-dibromoacrylonitrile (2l).
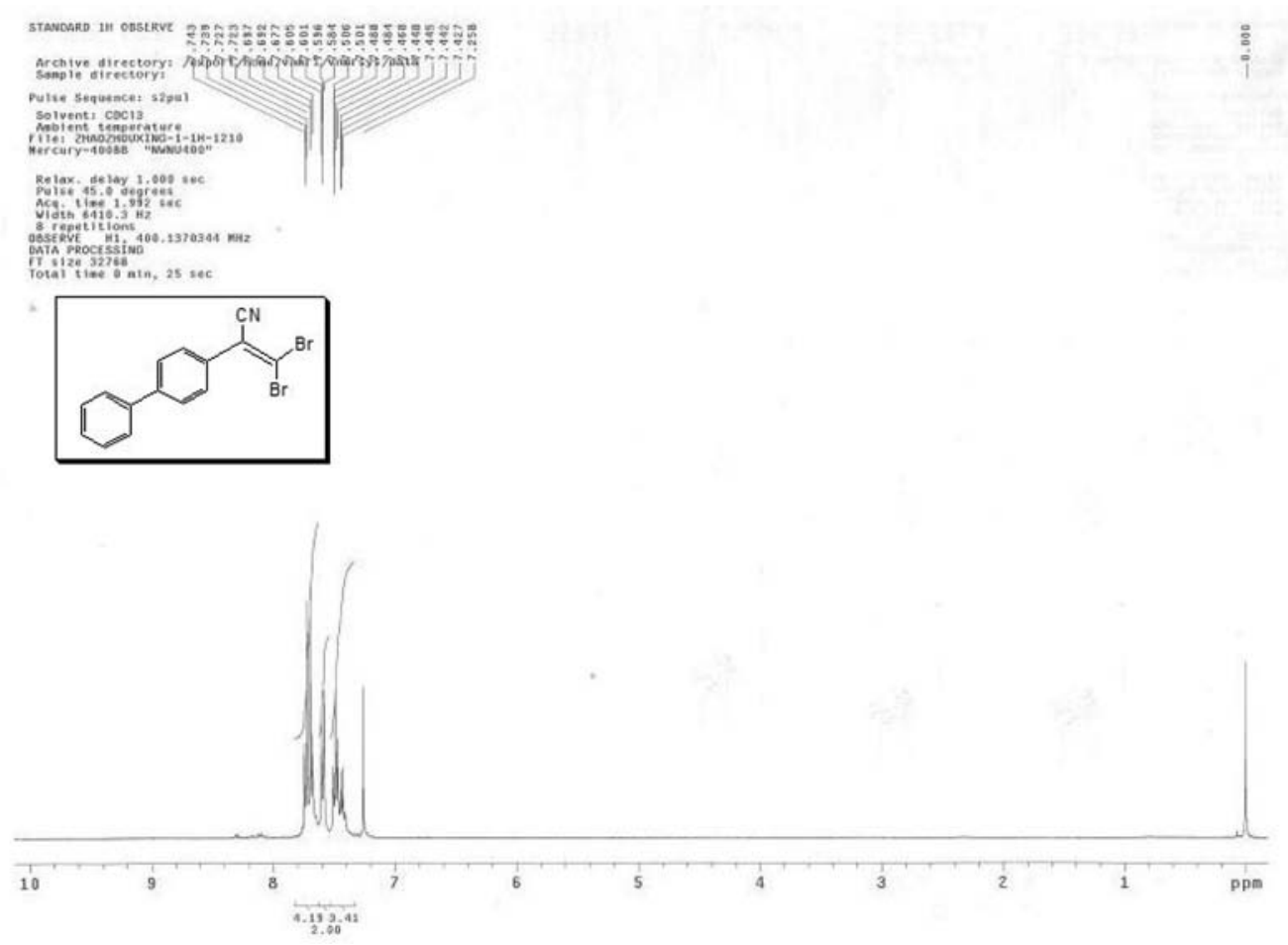

Figure S77. ${ }^{1} \mathrm{H}$ NMR spectrum of 2-(4-biphenyl)-3,3-dibromoacrylonitrile (2l). 


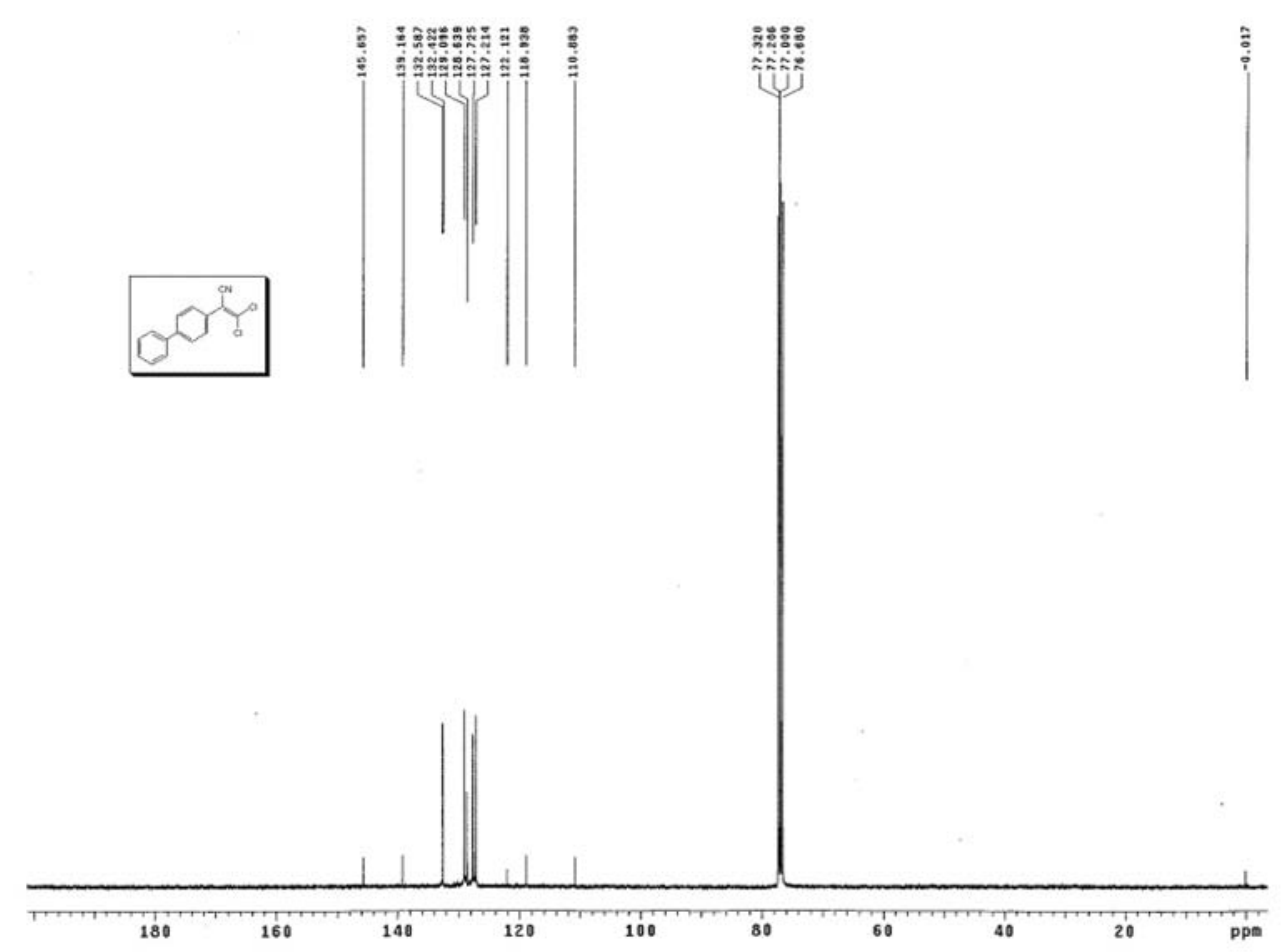

Figure S78. ${ }^{13} \mathrm{C}$ NMR spectrum of 2-(4-biphenyl)-3,3-dibromoacrylonitrile (2l).

\section{1,3-Bis(2,2-dibromo-1-cyanovinyl)benzene (2m)}

IR (KBr) $v_{\max } / \mathrm{cm}^{-1}: 3055,2923,2200,1636,1479,1433,1102,713,514 ;{ }^{1} \mathrm{H}$ NMR $\left(\mathrm{CDCl}_{3}, 400 \mathrm{MHz}\right): \delta 7.75-7.42(\mathrm{~m}$, $4 \mathrm{H}, \mathrm{Ar}-\mathrm{H}) ;{ }^{13} \mathrm{C} \mathrm{NMR}\left(\mathrm{CDCl}_{3}, 100 \mathrm{MHz}\right): \delta 133.3,132.4,132.3,132.2,131.5,128.5,128.4$.

\section{Digilab Merlin}

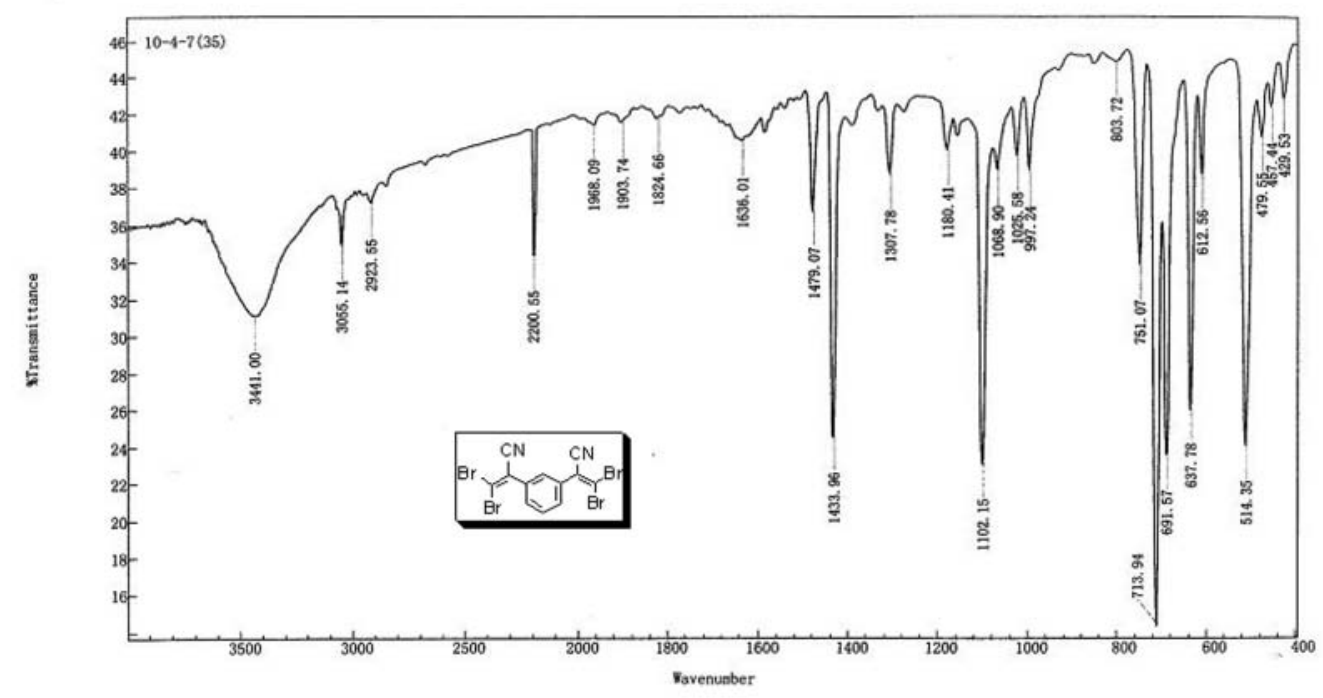

Figure S79. IR spectrum of 1,3-bis(2,2-dibromo-1-cyanovinyl)benzene (2m). 


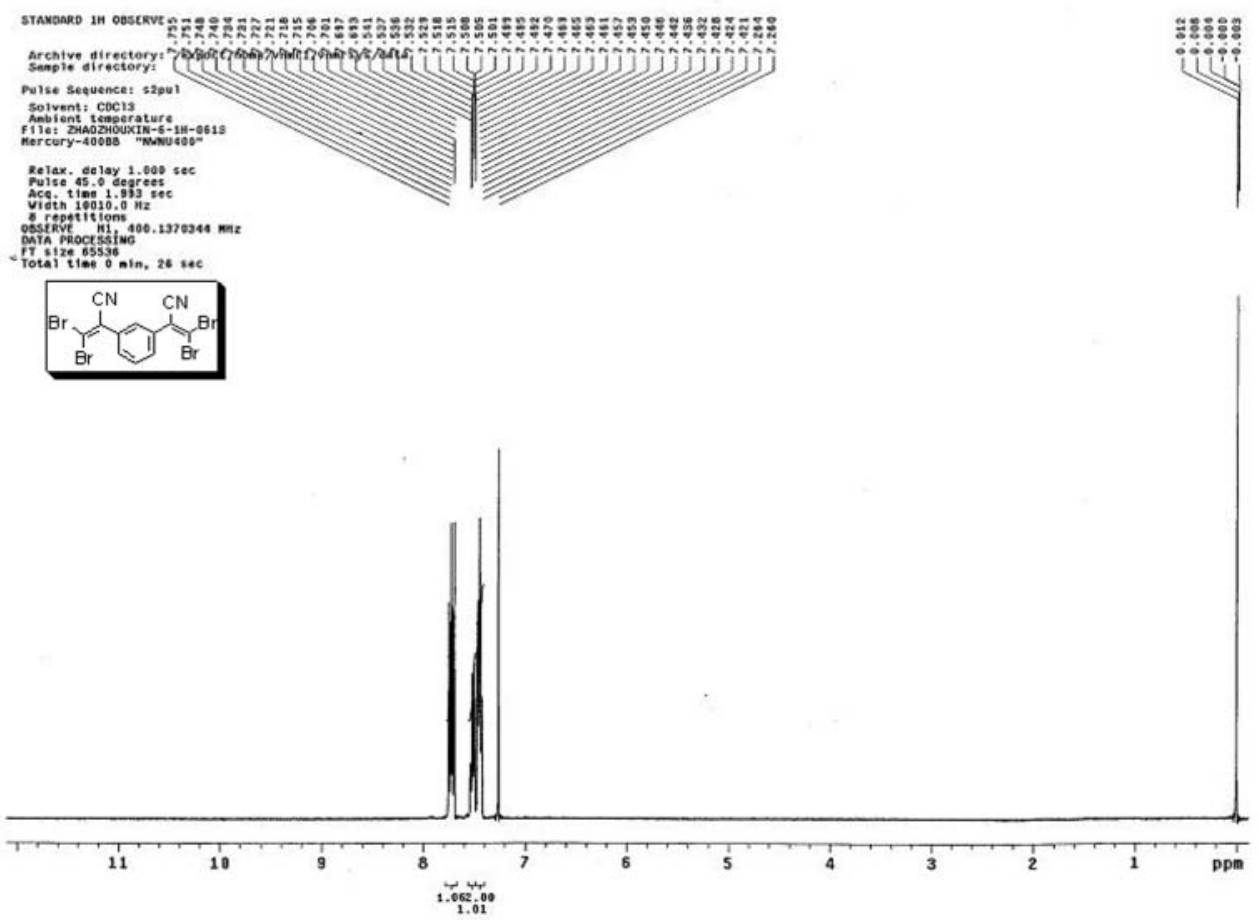

Figure S80. ${ }^{1} \mathrm{H}$ NMR spectrum of 1,3-bis(2,2-dibromo-1-cyanovinyl)benzene (2m).
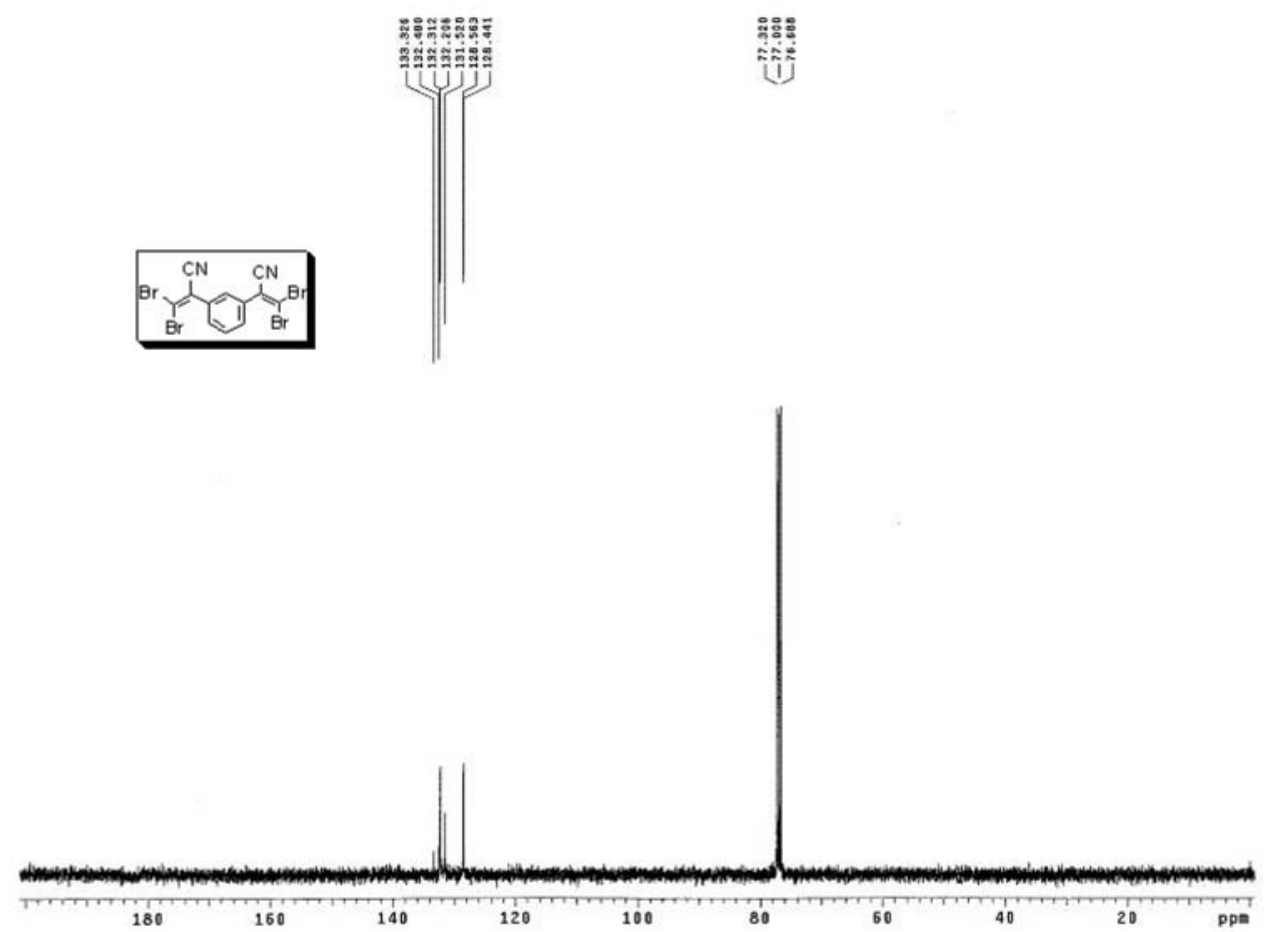

Figure S81. ${ }^{13} \mathrm{C}$ NMR spectrum of 1,3-bis(2,2-dibromo-1-cyanovinyl)benzene (2m). 
2-(Furan-2-yl)-3,3-dibromoacrylonitrile (2n)

$\mathrm{IR}(\mathrm{KBr}) \mathrm{v}_{\max } / \mathrm{cm}^{-1}: 3153,2227,1634,1534,1476,1030,850,750 ;{ }^{1} \mathrm{H} \mathrm{NMR}\left(400 \mathrm{MHz}, \mathrm{CDCl}_{3}\right): \delta 7.58(\mathrm{~d}, 1 \mathrm{H}, J 1.6 \mathrm{~Hz}$, Fu-H), 7.08 (d, $1 \mathrm{H}, J 4.0 \mathrm{~Hz}, \mathrm{Fu}-\mathrm{H}), 6.54$ (dd, $1 \mathrm{H}, J 3.6 \mathrm{~Hz}, J$ 1.6Hz, Fu-H); ${ }^{13} \mathrm{C}$ NMR $\left(\mathrm{CDCl}_{3}, 100 \mathrm{MHz}\right): \delta 145.9,144.2$, $115.3,114.5,113.5,112.1,103.7$.

\section{Digilab Merlin}

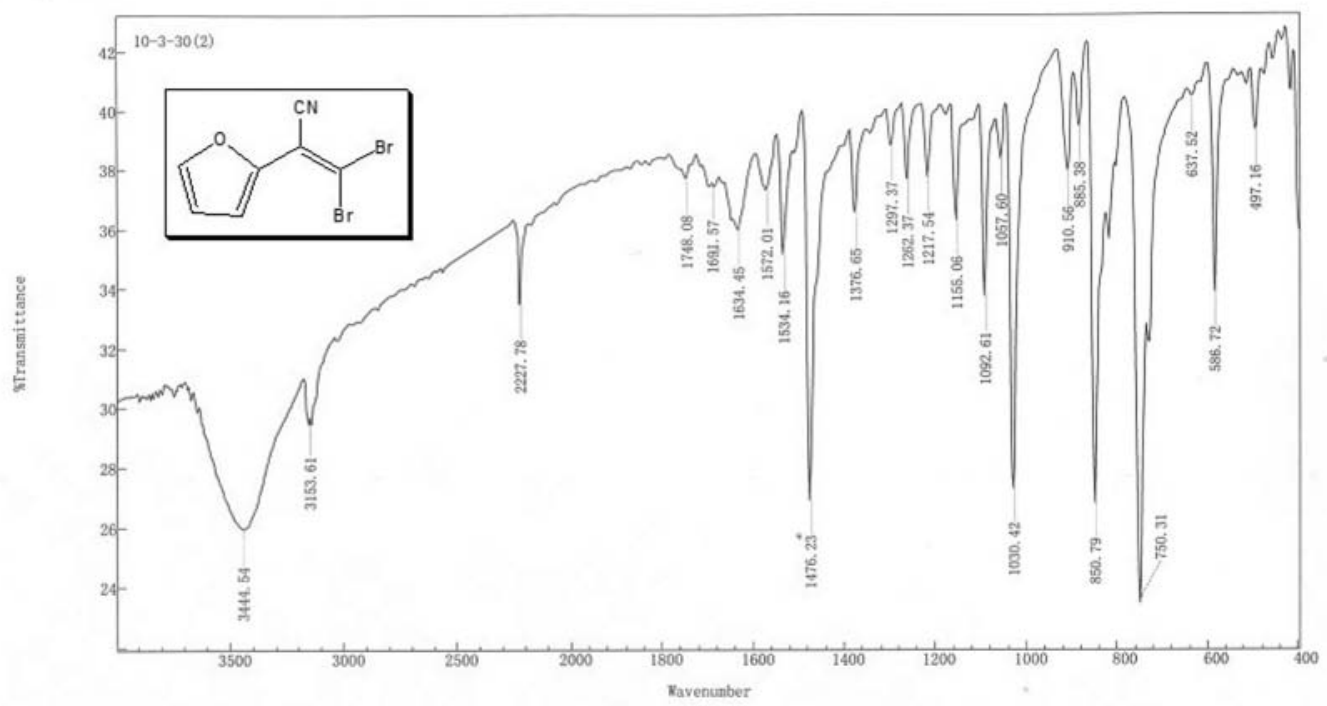

Figure S82. IR spectrum of 2-(furan-2-yl)-3,3-dibromoacrylonitrile (2n).

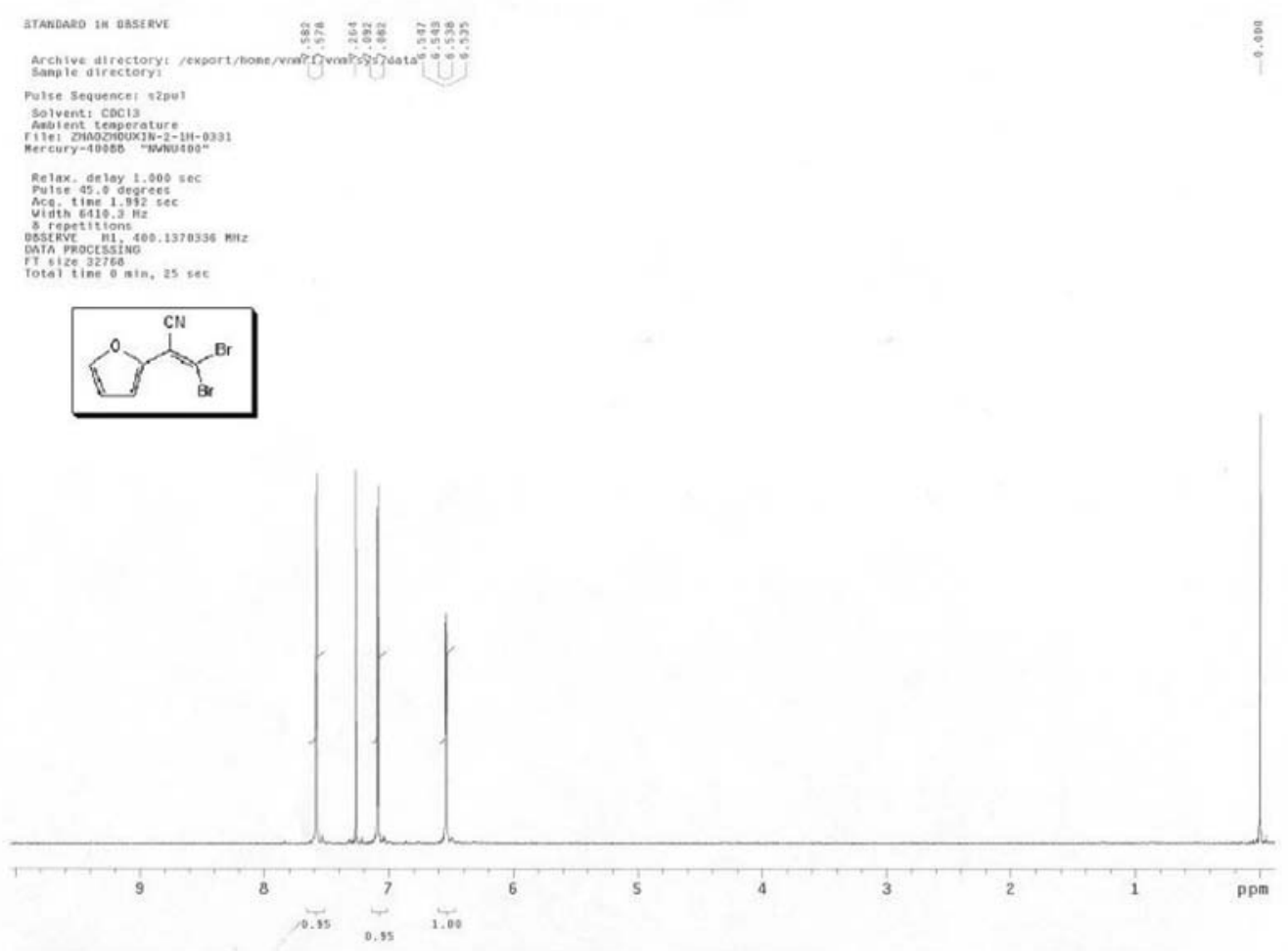

Figure S83. ${ }^{1} \mathrm{H}$ NMR spectrum of 2-(furan-2-yl)-3,3-dibromoacrylonitrile (2n). 

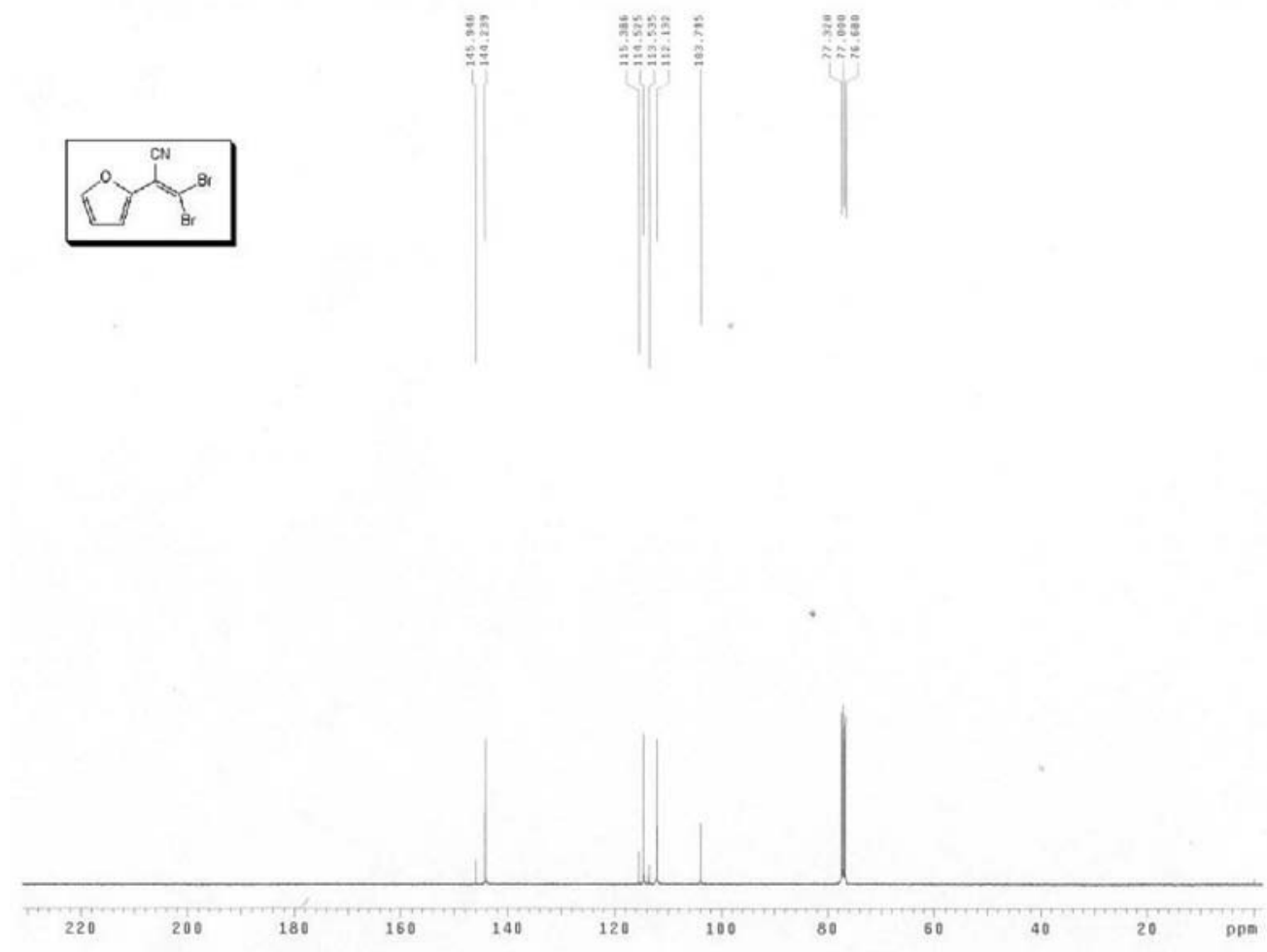

Figure S84. ${ }^{13} \mathrm{C}$ NMR spectrum of 2-(furan-2-yl)-3,3-dibromoacrylonitrile (2n). 